\title{
Propriedades Topológicas do Espaço das Assinaturas de um Anel Semilocal
}

\author{
Marcello Fidélis *
}

Orientadora: Profa. Dra. Ires Dias

Dissertação apresentada ao Instituto de Ciências Matemáticas e de Computação - ICMC-USP, como parte dos requisitos para obtenção do título de Mestre em Matemática.

USP - São Carlos

Fevereiro/2001

\footnotetext{
"Este trabalho contou com apoio financeiro da CAP
} 
A Comissão Julgadora:

Profa. Dra. Ires Dias

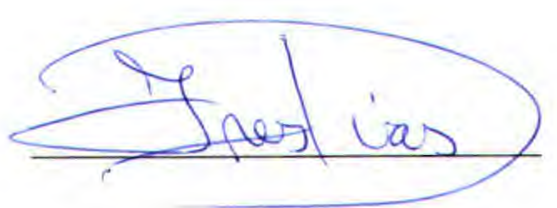

Prof. Dr. Vitor de Oliveira Ferreira

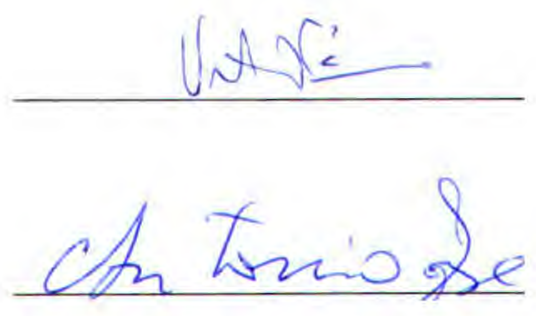

Prof. Dr. Antonio José Engler 


\section{Agradecimentos}

Primeiramente agradeço a Deus pela vida. À Prof ${ }^{a}$. Dr ${ }^{a}$. Ires Dias, por sua dedicada e paciente orientação, por sua amizade e incentivo a todo momento e ao seu filho Alexandre.

Quero também agradecer aqueles que tornaram minha vida mais agradável durante minha estadia em São Carlos:

- Minha sala de pós-graduação, repleta de amigos: Vera, Maria Alice, Daniela, Andréa, José, Luciene, Silvia e meu amigo desde a graduação Miguel.

- Meus companheiros de pós-graduação: Caniz, Luciana Martins \& Brito, Raimundo, Kurokawa, Carlos \& Glória, Alexandre \& Claudinha, Hilde, Eliris, Rubinha, Helton, Márcio, Sérgio, Esdras, Lee, Daniel Viais e Daniel Mancini.

- Aos professores que contribuiram para com minha formação e/ou com amizade. Em especial Valdir, Ires, Ladeira, Hildebrando, Hermínio, Wagner, Conde, Janey, Janete, Maria do Carmo, Zani, Pires, Joachin, Mirinha, Paulo da Veiga, Plácido, Hermano, Cidinha, Gaspar, Dide, Neide Franco, Rosane Minghim, Marcelo Saia, Daniel, Edna, Roberta, Sandra, Vitor, Alexandre e Biasi.

- Aos funcionários que sempre apoiaram em suas respectivas funções e, às vezes, além delas: Marília, Beth, Laura, Zé Angelo, Dolores, Pedro, Ana e Sandra.

- Aos professores da UEM: Aninha, Marcelo Hernanedes, Vitor Hugo, Regilene \& Tim, Ana Cláudia e Marcão.

- Aos meus amigos do quarto 20: Sam, Bloguinha e Véio; ao antigo quarto 19 e suas respectivas companheiras: Bigui \& Rosana, Pedro \& Ana Thé. 
- Aos meus amigos da minha república, a República Federativa dos Camarões que me aturaram por mais de um ano: Buda, Gorpo \& Jô. Aos amigos da República Toca das Virgens: Gauchinho, Clark, Tigrão e aos agregados Buck e Dartas.

- Aos alunos de graduação pelo companheirismo e amizade: Ronaldinho, Alex, Fabio, Kelli, Mariana, Luci, Grazielle, Aline, Josias, Flávia, Fabrizio, Érica e Benito.

- Aos amigos de momentos inesquecíveis: Patrícia, Anderson Manzoli \& Vanda, Perdido \& Telma, Maura, Rogério, Karinão, Ovo, Elisângela e Flávia (Loira) Franco.

- Agradeço as minhas famílias, que contribuiram para a formação do meu caráter e que moram no meu coração: Minha mãe D. Rita, minha Vó Irene (in memorium), meus irmãos Alexandre, Renata e Leandro. À Família Mota: "Seo" Silvio, D. Cida, Anderson, Thais, Thaiane e Thiciane. À Familia Takao: D. Emika (Okaa-san), KenSan, Miki, Lika e Eika.

- Ao querido Prof. Farid por ter aberto meus olhos para a linda Pérola.

$\odot$ Em especial para a namorada mais carinhosa do mundo: ma très chere Paula.

Também agradeço aqueles que direta ou indiretamente colaboraram e que por ventura (muito provavelmente) eu possa não ter lembrado.

Muito obrigado. 


\section{Resumo}

Neste trabalho apresentamos a equivalência entre a Propriedade da Aproximação Forte, Propriedade da Aproximação Fraca e Propriedade de Hasse-Minkowiski do espaço das assinaturas de um anel semilocal, sem assumirmos que 2 é invertível no anel. Tal estudo é baseado nos trabalhos de A. Rosenberg e R. Ware em [RW] e nas observações de M. Knebush em [Knel]. 


\begin{abstract}
In this work we show the equivalence between Strong Aproximation Property, Weak Aproximation Property and Hasse-minkowiski Property of the space of signatures of a semilocal ring, without assumption that 2 is invertible in the ring. Such study is based on the works of A. Rosenberg and R. Ware in [RW] and remarks of M. Knebush in [Kne1].
\end{abstract}




\section{Índice}

$\begin{array}{ll}\text { Introdução } & 1\end{array}$

1 Preliminares $\quad 3$

1.1 Álgebra Comutativa ....................... 3

1.2 Topologia de Zariski . . . . . . . . . . . . . . . 9

2 Espaços Bilineares e Quadráticos $\quad 13$

2.1 Definições . . . . . . . . . . . . . . . . . . . . . 13

2.2 Extensão e Contração de Espaços . . . . . . . . . . . . . . . 17

2.3 Subespaços . . . . . . . . . . . . . . . . . . . . . . 19

2.4 Bases Relacionadas . . . . . . . . . . . . . . . . 26

2.5 Espaços Metabólicos e Hiperbólicos . . . . . . . . . . . . . . . 30

2.6 Cancelamento de Witt . . . . . . . . . . . . . 37

3 O Anel de Witt $\quad 41$

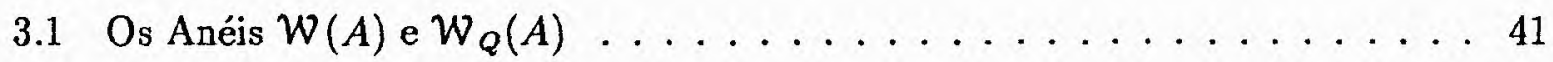

3.2 Geradores de $\mathcal{W}(A) \ldots \ldots \ldots \ldots \ldots \ldots \ldots$

3.3 Os ideais primos de $\mathcal{W}(A) \ldots \ldots \ldots \ldots \ldots \ldots$

3.4 Nilpotência e Torção . . . . . . . . . . . . . . . . . 52

3.5 Resultados sobre Assinaturas . . . . . . . . . . . . 55

4 Equivalências Topológicas do Espaço das Assinaturas $\quad 59$

4.10 espaço das assinaturas $\mathcal{A} s s(A) \ldots \ldots \ldots \ldots \ldots$

4.2 Propriedades Topológicas . . . . . . . . . . . . . . 62

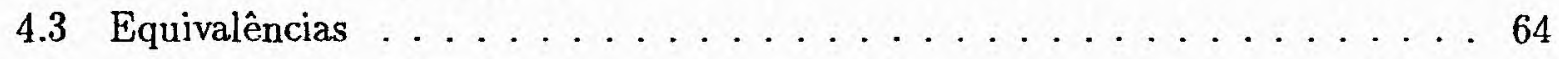

$\begin{array}{ll}\text { Bibliografia } & \mathbf{7 3}\end{array}$ 


\section{Introdução}

Acredita-se que o estudo das formas quadráticas tenha surgido na Babilônia e, até 1936, o mesmo era feito com formas quadráticas sobre o corpo dos números reais, o corpo dos números complexos ou o anel dos inteiros. Em 1937, Ernst Witt revolucionou o estudo das formas quadráticas. Em seu trabalho ele modificou o estudo das formas quadráticas estudando o conjunto de todas as formas quadráticas sobre um corpo genérico com característica distinta de 2, ao invés de uma forma particular. Este conjunto, ele repartiu em classes de equivalência e construiu um objeto algébrico, o principal de toda a teoria, hoje connhecido como o Anel de Witt. No entanto passaram-se quase 30 anos para que a importância deste estudo fosse revelada por Albrecht Pfister com seus teoremas de estruturas, criando a teoria "algébrica" das formas quadráticạs. Desde então o estudo da teoria algébreica das formas quadráticas se resume no estudo da estrutura do Anel de Witt.

Em [KRW1], M. Knebush, A. Rosenberg e R. Ware apresentam resultados sobre a estrutura de anéis de Witt como quocientes de anéis de grupo abelianos. Com estes resultados, obtemos a classificação dos ideais primos do Anel de Witt, $\mathcal{W}(A)$, das formas bilineares sobre um anel semilocal. $O$ conceito de assinatura está relacionado ao conjunto dos ideais primos de $\mathcal{W}(A), \operatorname{Spec}(\mathcal{W}(A))$ que é um espaço topológico com a topologia de Zariski. Paralelamente a esta definição define-se a topologia de Harrison para $\mathcal{A} s s(A)$, o conjunto das assinaturas de $A$.

Em 1972, R. Elman e T. Lam, no artigo [E-L], indroduziram uma propriedade topológica em $\mathcal{A} s s(A)$, para o caso que $A$ é um corpo, chamada Propriedade da Aproximação Fraca (PAF). Em [E-L] e independentemente por A. Prestel em [Pre], foi introduzida a Propriedade de Hasse-Minkowiski (PHM), também para o caso de corpos. Em 1973, M. Knebush, A. Rosenberg e R. Ware em [KRW2] introduziram a chamada Propriedade da Aproximação Forte (PAFF), para o caso em que $A$ é um anel semilocal.

Em [E-L], R. Elman e T. Lam mostraram que se $A$ é um corpo formalmente real, então 
(PAF) e (PAFF) são equivalentes e, se além disso, $A$ também é pitagoreano, então estas duas propriedades são também equivalentes à (PHM). Em [RW], A. Rosenberg e R. Ware mostraram as equivalências de (PAFF), (PAF) e (PHM) para o caso em que $A$ é um anel semilocal, com 2 invertível em $A$.

Neste trabalho, usando as técnicas desenvolvidas por M. Knebush em [Knel] mostraremos a mesma equivalência obtida em [RW] sem assumir 2 invertível em $A$.

Para tanto, nos capítulos I e II, apresentaremos alguns resultados de álgebra comutativa, topologia de Zariski e noçoes básicas de espaços bilineares e quadráticos sobre anéis semilocais, necessários à compreensão do restante do trabalho.

O capítulo III contém resultados sobre a estrutura do anel de Witt, $\mathcal{W}(A)$, dos espaços bilineares sobre um anel semilocal $A$. Mais precisamente, comtém a caracterização dos geradores de $\mathcal{W}(A)$ e a definição de $\mathcal{W}_{Q}(A)$, o anel dos espaços quadráticos sobre $A$ e sua relação com $\mathcal{W}(A)$. Alguns resultados sobre assinaturas, elementos de torção e nilpotentes de $\mathcal{W}(A)$ são também apresentados neste capítulo.

O capítulo IV consite do que nos propomos a desenvolver no projeto, ou seja, demonstrax a equivalência entre (PAF), (PAFF) e (PHM) em $\mathcal{A} s$ s ( $A$ ) quando $A$ é um anel semilocal sem impormos 2 invertível no anel, usando as técnicas desenvolvidas por $\mathrm{M}$. Knebush. $\mathrm{Na}$ primeira seção mostramos que $\mathcal{A s s}(A)$ é um espaço topológico compacto, de Hausdorff e totalmente desconexo, estabelecemos a relação entre a topologia de Zariski em $\operatorname{Spec}(\mathcal{W}(A))$ e a topologia de Harrison em $\mathcal{A} s s(A)$, generalizando para anéis a demonstração de W. Scharlau em [Sch], onde o mesmo resultado fora obtido para corpos. Na segunda seção definimos as propriedades (PAF), (PAFF) e (PHM) e apresentamos mais propriedades equivalentes às mesmas. Finalmente, na última seção apresentamos a generalizạ̧ãa dos resultados obtidos em $[\mathrm{RW}]$ mostrando a equivalência das propriedades citadas acima. 


\section{Capítulo 1}

\section{Preliminares}

Neste capítulo apresentaremos alguns fatos básicos de álgebra comutativa, bem como a definição e algumas propriedades da Topologia de Zariski para um anel comutatjvo $A$, necessários para o desenvolvimento deste trabalho. Algumas demonstrações serão omitidas, as quais podem ser encontradas na literatura, como por exemplo em [A-M]. No que segue e nos demais capítulos, $A$ denotará sempre um anel comutativo com elemento identidade 1 . Indicaremos por $S p e c(A)$ o conjunto dos ideais primos de $A$, por $S p m(A)$ o conjunto dos ideais maximais de $A$, por $\mathrm{J}(A)$ o radical de Jacobson de $A$, por $r(\mathfrak{a})$ o radical do ideal a e $A^{\star}$ o grupo das unidades de $A$. Assumiremos, também que todo $A$-módulo será unitário e que todo homomorfismo de anéis leva elemento identidade em elemento identidade. $O$ símbolo $\cong$ será usado para denotar isomorfismos entre estruturas algébricas.

\section{1 Álgebra Comutativa}

Os próximos resultados serão usados frequentemente no decorrer deste trabalho. O primeiro deles é uma caracterização de $\partial(A)$.

Proposição 1.1 Um elemento $\alpha \in A$ está em $\partial(A)$ se, e somente se, $1-\alpha \beta \in A^{\star}$, para todo $\beta \in A$.

Dem.: Ver (1.9) de [A-M].

Proposição 1.2 Sejam a um ideal de A contido em $\mathrm{g}(A)$ e $\alpha \in A$. Então $\bar{\alpha}=\alpha+\mathfrak{a}$ é uma unidade em $A / a$ se, e somente se, $\alpha$ é uma unidade em $A$. 
Dem.: Se $\alpha$ é uma unidade em $A$, então é imediato que $\alpha+\mathfrak{a}$ é invertível em $A / \mathfrak{a}$. Reciprocamente, se $\alpha+\mathfrak{a} \in(A / \mathfrak{a})^{\star}$, então existe $\beta \in A$ tal que $(\alpha+\mathfrak{a})(\beta+\mathfrak{a})=1+\mathfrak{a}$, ou seja, $1-\alpha \beta \in \mathfrak{a} \subseteq \partial(A)$. De (1.1) temos $\alpha \beta \in A^{\star}$ e, conseqüentemente $\alpha \in A^{\star}$.

Para $\mathfrak{m} \in S p m(A)$, denotaremos por $A_{\mathfrak{m}}$ a localização de $A$ em $\mathfrak{m}$.

Proposição 1.3 Seja $\alpha \in A$. São equivalentes:

(i) $\alpha \in A^{\star}$;

(ii) $\frac{\alpha}{1} \in\left(A_{\mathfrak{m}}\right)^{\star}$, para todo $\mathrm{m} \in \operatorname{Spm}(A)$;

(iii) $\bar{\alpha} \neq \overline{0}$ em $A / \mathfrak{m}$, para todo $\mathfrak{m} \in \operatorname{Spm}(A)$.

Dem.: Imediata.

Definição 1.4 Dizemos que um anel comutativo $A$, com elemento identidade 1 é um anel semilocal se $\operatorname{Spm}(A)$ é finito, ou seja, se $A$ tem somente um número finito de ideais maximais.

Exemplo 1.5 Todo corpo é um anel semilocal, ou mais geralmente, todo produto direto finito de corpos é um anel semilocal. Da correspondência entre os ideais de $A$ e os ideais de um anel quociente, temos que toda imagem homomórfica de um anel semilocal é também semilocal. Mais ainda, se $A$ é um anel semilocal, então $A_{\mathfrak{p}}$ também o é, para todo p pertencente ao $\operatorname{Spec}(A)$.

Observação 1.6 Se $A$ é um anel semilocal, então podemos encontrar um inteiro $h \geq 1$ tal que $2 h-1$ e $4 h-1$ são unidades em $A$. De fato, basta escolher $h \geq 0$ como sendo o produto de todos os números primos ímpares que aparecem como característica dos corpos $A / \mathfrak{m}$, onde $\mathfrak{m} \in S p m(A)$, e $h=1$ nos casos em que característica de $A / \mathfrak{m}$ é 0 ou 2 . Por (1.3), $(2 h-1)(4 h-1)$ é uma unidade em $A$ se, e somente se $(2 h-1)(4 h-1) \not \equiv 0 \bmod \mathrm{m}$, para todo $\mathfrak{m} \in \operatorname{Spm}(A)$. Suponha que $\overline{(2 h-1)(4 h-1)}=\overline{0} \mathrm{em} A / \mathfrak{m}_{i}$, para algum $\mathfrak{m}_{i}$, então $(2 h-1)(4 h-1) \equiv 0 \bmod p_{i}$, onde $p_{i}$ é a caracteristica de $A / \mathfrak{m}_{i}$. Mas pela escolha de $h$, temos que $h \equiv 0 \bmod p_{i}$, o que implica que $(2 h-1)(4 h-1) \equiv 1 \bmod p_{i}$, uma contradição. Conseqüentemente existe um número inteiro $h \geq 0$ tal que $(2 h-1)(4 h-1) \in A^{\star}$. 
Proposição 1.7 Seja $A$ um anel semilocal com $\operatorname{Spm}(A)=\left\{\mathfrak{m}_{1}, \ldots, \mathfrak{m}_{r}\right\}$. Então

$$
\frac{A}{\partial(A)} \cong \frac{A}{\mathrm{~m}_{1}} \times \cdots \times \frac{A}{\mathrm{~m}_{r}} .
$$

Dem.: Segue diretamente de (1.10) de [A-M].

Sejam $A$ um anel e $M$ um $A$-módulo. Para um ideal a de $A$, denotamos por $M(\mathfrak{a})$ - $A / \mathfrak{a}$-módulo $M \otimes(A / \mathfrak{a}) \cong M /(\mathfrak{a} M)$, onde $\otimes$ denotará sempre $\otimes_{A}$. Para $\mathfrak{p} \in S p e c(A)$, denotamos por $M_{\mathfrak{p}} \circ A_{\mathfrak{p}}$-módulo $M \otimes A_{\mathfrak{p}}$. Um $A$-módulo $M$ é dito ser livre se $M$ admite uma base, ou seja, se existe um conjunto $\left\{x_{i} ; i \in \Gamma\right\}$ de elementos de $M$, tais que $M \cong \bigoplus_{i \in \Gamma} A x_{i}$.

Proposição 1.8 Seja $A=F_{1} \times \cdots \times F_{r}$ um produto direto finito de corpos. Se $M$ é um A-módulo livre de dimensão n sobre $A$, então $M \cong M_{1} \times \cdots \times M_{r}$ onde cada $M_{i}$ é um $F_{i}$-espaço vetorial de dimensão $n$, para $i=1, \ldots, r$.

Dem.: Segue diretamente do fato que o produto cartesiano comuta com a soma direta finita.

Proposição 1.9 Seja $A$ um anel comutativo, a um ideal de $A$ com a $\subseteq \mathcal{J}(A)$ e $M u m$ A-módulo. $S e\left\{x_{1}, \ldots, x_{n}\right\} \subseteq M$ são tais que $\left\{\overline{x_{1}}, \ldots, \overline{x_{n}}\right\}$ é uma base de $M(\mathfrak{a})$ sobre $A / \mathfrak{a}$, onde $\overline{x_{i}}=x_{i}+\mathfrak{a} M$, então $\left\{x_{1}, \ldots, x_{n}\right\}$ é uma base de $M$ sobre $A$.

Dem.: Seja $N=A x_{1}+\ldots+A x_{n} \subseteq M$. Desde que $\left\{\overline{x_{1}}, \ldots, \overline{x_{n}}\right\}$ é uma base de $M(\mathfrak{a})$ sobre $A / \mathfrak{a}$ e $N(\mathfrak{a})=(A / \mathfrak{a}) \overline{x_{1}}+\ldots+(A / \mathfrak{a}) \overline{x_{n}}$, temos que $M(\mathfrak{a})=N(\mathfrak{a})$, ou seja, $M / \mathfrak{a} M=N / \mathfrak{a} N$. Assim $M=N+\mathfrak{a} M$ e, desde que $\mathfrak{a} \subseteq \partial(A)$, do Lema de Nakayama temos que $M=N$, ou seja, $\left\{x_{1}, \ldots, x_{n}\right\}$ é um conjunto de geradores de $M$.

Se existe $i=1, \ldots, n$ tal que $x_{i} \in A x_{1}+\ldots+A x_{i-1}+A x_{i+1}+\ldots+A x_{n}$, então $\overline{x_{i}} \in(A / \mathfrak{a}) \overline{x_{1}}+\ldots+(A / \mathfrak{a}) \overline{x_{i-1}}+(A / \mathfrak{a}) \overline{x_{i+1}}+\ldots+(A / \mathfrak{a}) \overline{x_{n}}$, o que contradiz o fato de $\left\{\overline{x_{1}}, \ldots, \overline{x_{n}}\right\}$ ser uma base de $M(\mathfrak{a})$. Conseqüentemente, $\left\{x_{1}, \ldots, x_{n}\right\}$ é uma base de $M$ sobre $A$, como queríamos.

Seja $A$ um anel. Uma seqüência de $A$-módulos e $A$-homomorfismos

$$
\cdots \longrightarrow M_{i-1} \stackrel{f_{i}}{\longrightarrow} M_{i} \stackrel{f_{i+1}}{\longrightarrow} M_{i+1} \longrightarrow \cdots
$$


é dita ser exata em $M_{i}$ se $\operatorname{Im}\left(f_{i}\right)=\operatorname{Ker}\left(f_{i+1}\right)$. Se a seqüência é exata em cada $M_{i}$, então dizemos que ela é uma seqüência exata.

Uma seqüência exata do tipo $0 \longrightarrow M_{1} \stackrel{J}{\rightarrow} M_{2} \stackrel{g}{\rightarrow} M_{3} \rightarrow 0$, é chamada uma seqüência exata curta.

Dizemos que uma seqüência exata

$$
\cdots \rightarrow M_{i-1} \stackrel{f_{i}}{\rightarrow} M_{i} \stackrel{J_{i+1}}{\longrightarrow} M_{i+1} \longrightarrow \cdots
$$

cinde em $M_{i}$, se o submódulo $X=\operatorname{Im}\left(f_{i}\right)=\operatorname{Ker}\left(f_{i+1}\right)$ é um somando direto de $M_{i}$. No caso de uma seqüência exata curta $0 \longrightarrow X \longrightarrow Y \longrightarrow Z \longrightarrow 0$, obviamente temos que ela cinde em $X$ e $Z$. Se, além disso, a seqüência cinde em $Y$, dizemos apenas que a seqüência exata cinde.

Proposição 1.10 Se uma seqüência exata curta de A-módulos

$$
0 \rightarrow X \stackrel{f}{\rightarrow} Y \stackrel{g}{\rightarrow} Z \longrightarrow 0
$$

cinde, então $Y \cong X \oplus Z$.

Dem.: Desde que a seqüência exata $0 \rightarrow X \stackrel{f}{\rightarrow} Y \stackrel{g}{\rightarrow} Z \rightarrow 0$ cinde, temos que $X$ é um somando direto de $Y$, ou seja, $Y=X \oplus W$, para algum $A$-módulo $W$. Temos, também que $X=\operatorname{Im}(f)=\operatorname{Ker}(g)$. Assim, desde que $g$ é sobrejetor, temos

$$
\frac{Y}{\operatorname{Ker}(g)} \cong \operatorname{Im}(g)=Z \text {. }
$$

Portanto, $Z$ é isomorfo ao complementar de $X$ em $Y$, ou seja, $Z \cong W$. Assim $Y=X \oplus W \cong$ $X \oplus Z$, como queríamos.

Um $A$-módulo $P$ é dito ser um $A$-módulo projetivo se satisfaz uma das seguintes condições equivalentes:

(i) $P$ é somando direto de um A-módulo livre;

(ii) Toda seqüência exata curta de $A$-módulos $0 \rightarrow M \rightarrow N \rightarrow P \rightarrow 0$, cinde;

(iii) Para toda seqüência exata curta de $A$-módulos $0 \rightarrow X \rightarrow Y \rightarrow Z \rightarrow 0$, a seqüência de $A$-módulos

$$
0 \longrightarrow \operatorname{Hom}(P, X) \rightarrow \operatorname{Hom}(P, Y) \rightarrow \operatorname{Hom}(P, Z) \rightarrow 0
$$

é exata; 
(iv) Para todo diagrama de $A$-módulos e $A$-homomorfismos

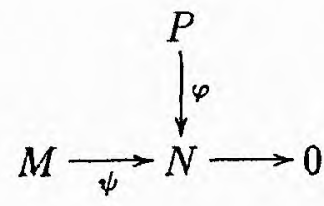

existe um levantamento de $\varphi, \varphi^{*}: P \longrightarrow M$, tal que o diagrama abaixo é comutativo, isto é, $\psi \circ \varphi^{*}=\varphi$.

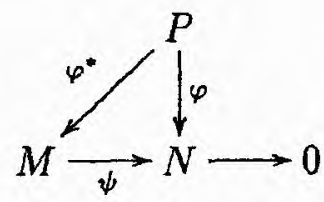

Não apresentaremos aqui a demonstração das equivalências que caracterizam um $A$ módulo projetivo, pois está fora dos objetivos de nosso trabalho mas, esta caracterização é clássica e pode ser encontrada, por exemplo, em [McD].

É imediato que todo $A$-módulo livre é também projetivo.

Se $M$ é um $A$-módulo projetivo finitamente gerado, e $\mathfrak{m} \in S p m(A)$, definimos o posto de $M$ módulo $\mathrm{m}$, como sendo a dimensão do $(A / \mathfrak{m})$-espaço vetorial $M(\mathfrak{m})$. Dizemos que $M$ é um $A$-módulo projetivo finitamente gerado de posto constante se a aplicação $\rho: \operatorname{Spm}(A) \longrightarrow \mathbb{Z}$, definida por $\rho(\mathfrak{m})=\operatorname{dim}_{A_{\mathfrak{m}}} M(\mathfrak{m})$ é constante.

A teoria algébrica das formas bilineares e quadráticas sobre anéis é desenvolvida na categoria dos módulos projetivos finitamente gerados e de posto constante. Finalizaremos esta seção mostrando que, sobre anéis semilocais, esta categoria coincide com a categoria dos módulos livres de dimensão finita. Para tanto, usaremos o seguinte resultado auxiliar.

Lema 1.11 Sejam $A$ um anel semilocal, $P$ um A-módulo projetivo finitamente gerado $\varepsilon$ $\mathfrak{a} \subseteq \partial(A)$ um ideal. Se $P(\mathfrak{a})$, é um $(A / \mathfrak{a})$-módulo livre, então $P$ é um A-módulo livre.

Dem.: Desde que $P(\mathfrak{a})$ é um $(A / \mathfrak{a})$-módulo livre e $P$ é finitamente gerado, então existe $m \in \mathbb{N}$ tal que $P(\mathfrak{a}) \cong(A / \mathfrak{a})^{m}=(A / \mathfrak{a}) \times \cdots \times(A / \mathfrak{a})$ ( $m$ vezes). Considerando as sobrejeções naturais $\pi$ de $P$ em $P(\mathfrak{a})$ e de $A^{m}$ em $(A / \mathfrak{a})^{m}$, temos o seguinte diagrama de A-módulos.

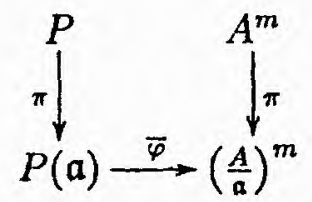


onde $\bar{\varphi}$ é um isomorfismo de $(A / \mathfrak{a})$-módulos e, portanto de $A$-módulos. Temos então o diagrama

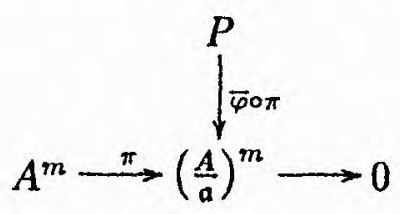

e, desde que $P$ é projetivo, temos que existe $\varphi: P \rightarrow A^{m}$ tal que o diagrama abaixo é comutativo

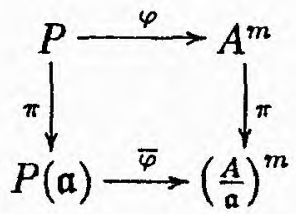

Agora, é suficiente mostrarmos que $\varphi$ é um isomorfismo. Desde que $\pi \circ \varphi=\bar{\varphi} \circ \pi$ é sobrejetor, temos que $\pi(\operatorname{Im}(\varphi))=(A / \mathfrak{a})^{m}$, ou seja, $\operatorname{Im}(\varphi)+\mathfrak{a} A^{m} \cong A^{m}$. Como $\mathfrak{a} \subseteq \mathcal{J}(A)$, do Lema de Nakayama temos que $\operatorname{Im}(\varphi) \cong A^{m}$, isto é, $\varphi$ é sobrejetor. Portanto temos a seqüência exata $0 \longrightarrow \operatorname{Ker}(\varphi) \longrightarrow P \longrightarrow A^{m} \longrightarrow 0$. Mas, $A^{m}$ é um $A$-módulo livre e, portanto projetivo, implicando em $P \cong \operatorname{Ker}(\varphi) \oplus A^{m}$. Assim $\operatorname{Ker}(\varphi)$ é um $A$-módulo finitamente gerado tal que $\frac{\operatorname{Ker}(\varphi)}{\mathfrak{a} \operatorname{Ker}(\varphi)} \subseteq \operatorname{Ker}(\bar{\varphi})=\{0\}$, pois $\bar{\varphi}$ é injetor.

Logo $\operatorname{Ker}(\varphi) \cong \mathfrak{a} \operatorname{Ker}(\varphi)$ e, novamente pelo Lema de Nakayama, temos que $\operatorname{Ker}(\varphi)=\{0\}$, completando a demonstração.

Teorema 1.12 Todo A-módulo projetivo finitamente gerado e de posto constante sobre um anel semilocal $A$ é livre.

Dem.: Sejam $A$ um anel semilocal com $\operatorname{Spm}(A)=\left\{\mathfrak{m}_{1}, \ldots, \mathfrak{m}_{r}\right\}$ e $P$ um $A$-módulo projetivo finitamente gerado e de posto constante, com $n=\rho(P)$, ou seja, $n=\operatorname{dim}_{A / \mathrm{m}_{i}}\left(P\left(\mathrm{~m}_{i}\right)\right)$, para todo $\mathrm{m}_{i} \in \operatorname{Spm}(A), i=1, \ldots$, r. Para $\bar{P}=\frac{P}{\partial(P)}$, temos

$$
\bar{P} \cong \frac{P}{\mathfrak{m}_{1} P} \oplus \cdots \oplus \frac{P}{\mathfrak{m}_{r} P} \cong\left(\frac{A}{\mathfrak{m}_{1}}\right)^{n} \oplus \cdots \oplus\left(\frac{A}{\mathfrak{m}_{r}}\right)^{n} \cong\left(\frac{A}{\mathfrak{m}_{1}} \times \cdots \times \frac{A}{\mathfrak{m}_{r}}\right)^{n}
$$

Logo $\bar{P}$ é um $\bar{A}=(A / \mathcal{J}(A))$-módulo livre de dimensão $n$ e, do lema anterior, segue que $P$ é um $A$-módulo livre. 


\subsection{Topologia de Zariski}

Nesta seção apresentaremos a topologia de Zariski para o anel $A$ e algumas propriedades topológicas relevantes para o desenvolvimento de nosso trabalho.

Dado um conjunto não vazio $Y$, uma subbase $\mathcal{S}$ para uma topologia no conjunto $Y$ é uma coleção de subconjuntos de $Y$ tal que a união é igual a $Y$. A topologia gerada pela subbase $\mathcal{S}$ é a coleção $\mathcal{T}$ de todas as uniões de interseções finitas de elementos de $\mathcal{S}$.

Um espaço topológico $Y$ é dito ser totalmente desconexo se seus únicos subconjuntos conexos são os subconjuntos unitários. Se $W$ é um subconjunto aberto e fechado do espaço topológico $Y$, diremos que $W$ é um subconjunto fechaberto de $Y$.

Proposição 1.13 Seja $Y$ um espaço topológico, se quaisquer dois pontos de $Y$ podem ser separados por fechabertos, então $Y$ é totalmente desconexo.

Dem.: Suponhamos, por absurdo que $Y$ admita um subconjunto conexo $C$ com mais de um elemento. Sejam $x, y \in C, x \neq y$. De acordo com a hipótese, existem $U_{x}, U_{y}$ fechabertos tais que $x \in U_{x}, y \in U_{y}$ e $U_{x} \cap U_{y}=\varnothing$. Assim, $C=\left(U_{x} \cap C\right) \cup\left(U_{y} \cap C\right)$ é uma cisão não trivial de $C$, absurdo pois $C$ é conexo. Logo os únicos conexos de $Y$ são os subconjuntos unitários.

Suponha que os conjuntos unitários do espaço topológico $Y$ são fechados. Então $Y$ é dito ser regular se para cada $x \in Y$ e $B \subseteq Y$ fechado tal que $x \notin B$, existem subconjuntos abertos disjuntos contendo $x$ e $B$, respectivamente. O espaço topológico é dito ser normal se para cada par $B, C$ de subconjuntos de $Y$, fechados disjuntos, existem subconjuntos abertos disjuntos contendo $B$ e $C$, respectivamente.

Proposição 1.14 Todo espaço topológico $Y$ de Hausdorff compacto é normal.

Dem.: Ver [Mun], Chapter 4, Theorem 2.4.

Sejam $A$ um anel e $X=S p e c(A)$, o conjunto dos ideais primos de $A$. Para qualquer subconjunto $B$ de $A$, denotamos por $V(B)$ o conjunto dos ideais primos de $A$ que contém $B$. É imediato que se $\mathbf{a}=(B)$, onde $(B)$ denota o ideal gerado pelo conjunto $B$, temos $V(\mathfrak{a})=V(B)$. Quando $B$ consiste de um único elemento $\alpha$, escrevemos $V(\alpha)$ ao invés de $V(\{\alpha\})$. Assim $V(\alpha)=V((\alpha))$. 
A aplicação $B \mapsto V(B)$ é decrescente com respeito à inclusão em $A$ e em $X$. Mais ainda, valem as seguintes propriedades:

(1) $V(0)=X, \quad V(1)=\varnothing ;$

(2) $V\left(\bigcup_{i \in I} B_{i}\right)=V\left(\sum_{i \in I} B_{i}\right)=\bigcap_{i \in I} V\left(B_{i}\right)$, para qualquer família $\left(B_{i}\right)_{i \in I}$ de subconjuntos de $A$;

(3) $V\left(\mathfrak{a} \cap \mathfrak{a}^{\prime}\right)=V\left(\mathfrak{a} \mathfrak{a}^{\prime}\right)=V(\mathfrak{a}) \cap V\left(\mathfrak{a}^{\prime}\right)$, para qualquer par de ideais $\mathfrak{a}, \boldsymbol{a}^{\prime}$ em $A$.

As propriedades (1) e (2) são de demonstração imediata; por outro lado, (3) significa que, um ideal primo $\mathfrak{p}$ de $A$ contém um dos ideais $\mathfrak{a}$ ou $\mathfrak{a}^{\prime}$ se, e somente se o mesmo contém $a a^{\prime}$ ou $a \cap a^{\prime}$. A segunda parte de (1) possui a seguinte recíproca: se a é um ideal de $A$ tal que $V(\mathfrak{a})=\varnothing$, então $\boldsymbol{a}=A$, pois não existe ideal maximal que o contenha. Finalmente, se a é um ideal de $A$ e $r(\mathfrak{a})$ é seu radical, então:

(4) $V(\mathfrak{a})=V(r(\mathfrak{a}))$.

A propriedade (4) segue de (1.14) de $[A-M]$.

As propriedades (1) até (3) mostram que os subconjuntos $V(B)$ de $X$ satisfazem os axiomas dos conjuntos fechados de uma topologia. Assim, temos que o conjunto dos ideais primos de $A$, é um espaço topológico, cujos fechados são os subconjuntos $V(B)$, onde $B$ percorre $\mathcal{P}(A)$. Esta topologia é chamada de topologia espectral ou topologia de Zariski em $\operatorname{Spec}(A)$.

Seja $X=\operatorname{Spec}(A)$. Para todo $\alpha \in A$ denotamos por $U(\alpha)$ o conjunto dos ideais primos de $A$ que não contém $\alpha$, isto é $U(\alpha)=\{\mathfrak{p} \in \operatorname{Spec}(A) ; \alpha \notin \mathfrak{p}\}$. Então $U(\alpha)=X-V(\alpha)$, ou seja $U(\alpha)$ é um conjunto aberto de $X$.

Por (2), qualquer subconjunto fechado de $X$ é interseção de subconjuntos fechados da forma $V(\alpha)$, o que mostra que os $U(\alpha)$ 's, com $\alpha \in A$, formam uma base para a topologia espectral em $X$. Mais ainda, segue das propriedades acima que:

$$
U(0)=\varnothing, \quad U(1)=\operatorname{Spec}(A)=X
$$

e mais geralmente, $U(\alpha)=X$ para cada $\alpha \in A^{\star}$ e $U(\alpha \beta)=U(\alpha) \cap U(\beta)$, para todo $\alpha, \beta \in A$. 
Para todo subconjunto $Y$ de $X=S p e c(A)$, denotamos por $\mathcal{J}(Y)$ a interseção de todos os ideais primos de $A$ que estão em $Y$. Então $\mathcal{J}(Y)$ é um ideal de $A$ e a aplicação $Y \mapsto \mathcal{J}(Y)$ é decrescente com respeito à inclusão em $X$ e em $A$.

Valem as seguintes relações

$$
\mathcal{J}(\varnothing)=A, \quad \mathcal{J}\left(\bigcup_{\lambda \in \Lambda} Y_{\lambda}\right)=\bigcap_{\lambda \in \Lambda} \mathcal{T}\left(Y_{\lambda}\right)
$$

para toda família $\left(Y_{\lambda}\right)_{\lambda \in \Lambda}$ de subconjuntos de $X$. Mais ainda:

Proposição 1.15 Sejam $A$ um anel, a um ideal de $A$ e $Y$ um subconjunto de $X=$ Spec(A).

(i) $V(a)$ é fechado em $X$ e $\mathcal{T}(Y)$ é um ideal de $A$ que é igual ao seu radical.

(ii) $\mathcal{J}(V(\mathfrak{a}))$ é o radical de a e $V(\mathcal{J}(Y))$ é o fecho de $Y$ em $X$.

(iii) As aplicações J e $V$ definem bijeções decrescentes, uma inversa da outra, entre os subconjuntos fechados de $X$ e o conjunto dos ideais de $A$ que são iguais a seu radical.

Dem.: Segue diretamente da definição da topologia de Zariski que $V(\mathfrak{a})$ é fechado em $X$. Sabemos também que $r(\mathcal{J}(Y))$ é a interseção de todos os ideais primos $\mathfrak{p}$ de $A$ que contém $\mathcal{J}(Y)$ (ver (1.4) de $[A-M])$. Em particular, como $\mathcal{J}(Y) \subseteq \mathfrak{p}$ para todo $\mathfrak{p} \in Y$, temos que, $r(\mathcal{J}(Y)) \subseteq \bigcap_{p \in Y} \mathfrak{p}=\mathcal{J}(Y)$. A outra inclusão é imediata, o que mostra $(i)$.

Usando novamente (1.4) de $[\mathrm{A}-\mathrm{M}]$ é imediato que $\mathcal{J}(V(\mathfrak{a}))=r(\mathfrak{a})$. Seja $V(B)$ um conjunto fechado tal que $Y \subseteq V(B)$, para algum $B \subseteq A$. Então cada ideal primop de $Y$ contém $B$, logo $B \subseteq \bigcap_{\mathfrak{p} \in Y} \mathfrak{p}=\mathcal{J}(Y)$ e, conseqüentemente $V(B) \supseteq V(\mathcal{J}(Y))$. Como $Y \subseteq V(\mathcal{J}(Y))$, temos que $V(\mathcal{J}(Y))$ está contido em todo fechado de $X$ que contém $Y$. Em particular $V(\mathcal{T}(Y)) \subseteq \bar{Y}$. A inclusão contrária é óbvia pois $Y \subseteq V(\mathcal{T}(Y))$, o que mostra (ii).

As afirmações de (iii) seguem diretamente de (ii).

Corolário 1.16 Se $\left(Y_{\lambda}\right)_{\lambda \in \Lambda}$ é uma familia de subconjuntos fechados de $X$, então $\mathcal{J}\left(\bigcap_{\lambda \in \Lambda} Y_{\lambda}\right)$ é o radical da soma dos ideais $\mathcal{J}\left(Y_{\lambda}\right)$, com $\lambda \in \Lambda$. 
Dem.: Da proposição anterior, item (iii), segue que J $\left(\bigcap_{\lambda \in \Lambda} Y_{\lambda}\right)$ é o menor ideal, igual ao seu radical, que contém $\mathcal{J}\left(Y_{\lambda}\right)$ para todo $\lambda \in \Lambda$. Assim $\sum_{\lambda \in \Lambda} \mathcal{J}\left(Y_{\lambda}\right) \subseteq \mathcal{J}\left(\bigcap_{\lambda \in \Lambda} Y_{\lambda}\right)$. Conseqüentemente, $r\left(\sum_{\lambda \in \Lambda} \mathcal{J}\left(Y_{\lambda}\right)\right) \subseteq r\left(\mathcal{J}\left(\bigcap_{\lambda \in \Lambda} Y_{\lambda}\right)\right)=\mathcal{J}\left(\bigcap_{\lambda \in \Lambda} Y_{\lambda}\right)$. Por outro lado, $r\left(\sum_{\lambda \in \Lambda} \mathcal{J}\left(Y_{\lambda}\right)\right)$ é um ideal, igual ao seu radical, e contém $\mathcal{J}\left(Y_{\lambda}\right)$ para todo $\lambda \in \Lambda$. Portanto, $\mathcal{J}\left(\bigcap_{\lambda \in \Lambda} Y_{\lambda}\right) \subseteq r\left(\sum_{\lambda \in \Lambda} \mathcal{J}\left(Y_{\lambda}\right)\right)$, como queríamos.

Corolário 1.17 Sejam $\mathfrak{a} e \mathfrak{b}$ ideais do anel A. São equivalentes:

(i) $V(\mathfrak{a}) \subseteq V(\mathbf{b})$;

(ii) $\mathbf{b} \subseteq r(\mathfrak{a})$;

(iii) $r(\mathfrak{b}) \subseteq r(\mathfrak{a})$.

Dem.: A equivalência $(i i) \Leftrightarrow$ (iii) é imediata.

Se $V(\mathfrak{a}) \subseteq V(\mathfrak{b})$, então

$$
r(\mathfrak{b})=\bigcap_{\mathfrak{p} \in V(\mathfrak{b})} \mathfrak{p} \subseteq \bigcap_{\mathfrak{p} \in V(\mathfrak{a})} \mathfrak{p}=r(\mathfrak{a})
$$

Reciprocamente, se $r(\mathfrak{b}) \subseteq r(\mathfrak{a})$, então de (1.15), (iii), temos que $V(r(\mathfrak{a})) \subseteq V(r(\mathfrak{b}))$, agora o resultado segue da propriedade (4) acima.

Corolário 1.18 Seja $\left(\alpha_{\lambda}\right)_{\lambda \in \Lambda}$ uma família de elementos de $A$. Para $\beta \in A$, $U(\beta) \subseteq \bigcup_{\lambda \in \Lambda} U\left(\alpha_{\lambda}\right)$ se, e somente se existe um inteiro $n>0$ tal que $\beta^{n}$ pertence ao ideal gerado pelos $\alpha_{\lambda}$, com $\lambda \in \Lambda$.

Dem.: A relação $U(\beta) \subseteq \bigcup_{\lambda \in \Lambda} U\left(\alpha_{\lambda}\right)$ é equivalente a $V(\beta) \supseteq \bigcap_{\lambda \in \Lambda} V\left(\alpha_{\lambda}\right)=V\left(\bigcup_{\lambda \in \Lambda} \alpha_{\lambda}\right)$. Agora o resultado segue de (1.17). 


\section{Capítulo 2}

\section{Espaços Bilineares e Quadráticos}

Apresentaremos neste capítulo a noção e alguns resultados básicos da teoria das formas bilineares e quadráticas sobre um anel semilocal $A$. Alguns destes resultados são mais gerais e valem para um anel comutativo com elemento identidade.

A teoria das formas bilineares e quadráticas sobre um anel $A$, é feita geralmente na categoria dos $A$-módulos projetivos finitamente gerados e de posto constante. Os principais resultados que apresentaremos neste trabalho envolvem espaços bilineares e quadráticos sobre aneis semilocais e, de (1.12) temos que neste caso, todo módulo projetivo finitamente gerado e de posto constante é livre. Assim, por conveniência de redação, trabalharemos desde o início com a categoria dos $A$-módulos livres de dimensão finita. Denotaremos esta categoria por $\mathcal{L}(A)$. No que segue, exceto menção contrária $A$ será sempre um anel semilocal, $\otimes$ significará sempre $\otimes_{A}$. Para cada $M \in \mathcal{L}(A)$, denotaremos por $M^{*} \circ A$-módulo dual $\operatorname{Hom}_{A}(M, A) \in \mathcal{L}(A)$ e todos os módulos considerados serão $A$-módulos.

\subsection{Definições}

Definição 2.1 O par $(M, b)$ consistindo de um módulo $M \in \mathcal{L}(A)$ e de uma forma bilinear simétrica $b: M \times M \rightarrow A$ é chamado de módulo bilinear sobre $A$. $O$ módulo bilinear $(M, b)$ é dito ser não singular, ou simplesmente um espaço bilinear, se a função $A$-linear $d_{b}: M \rightarrow M^{*}$, definida por $d_{b}(x)=b(x, \quad)$, para todo $x \in M$, é um isomorfismo de módulos. A função $d_{b}$ é chamada adjunta da forma bilinear $b$. Se o módulo $M$ tem dimensão $n$, ou seja, $M$ tem uma base com $n$ elementos, dizemos que o espaço bilinear $(M, b)$ tem dimensão $n$ e indicamos por $\operatorname{dim}(M, b)=n$, ou simplesmente por $\operatorname{dim}(M)=n$, 
ou ainda $\operatorname{dim}(b)=n$. Uma isometria entre dois módulos bilineares $\left(M_{1}, b_{1}\right)$ e $\left(M_{2}, b_{2}\right)$ é um isomorfismo de $A$-módulos $\varphi: M_{1} \rightarrow M_{2}$, que satisfaz $b_{1}(x, y)=b_{2}(\varphi(x), \varphi(y))$, para todo $x, y \in M_{1}$. Quando existe uma isometria entre $\left(M_{1}, b_{1}\right)$ e $\left(M_{2}, b_{2}\right)$ dizemos que os módulos bilineares sâo isométricos e denotamos por $\left(M_{1}, b_{1}\right) \simeq\left(M_{2}, b_{2}\right)$, ou simplesmente $b_{1} \simeq b_{2}$, ou ainda $M_{1} \simeq M_{2}$.

Se $(M, b)$ é um módulo bilinear sobre $A$ e $\left\{x_{1}, \ldots, x_{n}\right\}$ é uma base de $M$, então a forma bilinear $b$ é determinada pela matriz quadrada $\left(b_{i j}\right)=\left(b\left(x_{i}, x_{j}\right)\right), 1 \leq i, j \leq n$, pois para $x=\sum_{i=1}^{n} \alpha_{i} x_{i}$ e $y=\sum_{j=1}^{n} \beta_{j} x_{j}$ em $M$, temos que $b(x, y)=\sum_{i, j=1}^{n} b_{i j} \alpha_{i} \beta_{j}$. Reciprocamente, para cada matriz simétrica $n \times n,\left(b_{i j}\right)$, sobre $A$ obtemos uma forma bilinear simétrica sobre $M$ dada pela mesma fórmula descrita acima. É fácil ver que o módulo bilinear $(M, b)$ é não singular se, e somente se $\operatorname{det}\left(b_{i j}\right)$ é uma unidade em $\mathrm{A}$.

Chamaremos de determinante do espaço bilinear $(M, b)$ e denotaremos por $\operatorname{det}(b)$ o determinante da matriz $\left(b_{i j}\right)$. No que segue, identificaremos um elemento $x \in M$, com o vetor das coordenadas de $x$ em relação a uma dada base de $M$. Mais ainda, como um módulo $(M, b)$ é caracterizado por uma matriz quadrada $\left(b_{i j}\right)$, usaremos também a notação $b=\left(b_{i j}\right)$ para indicarmos a forma bilinear $b$.

Definição 2.2 Uma forma quạdrática em um módulo $M \in \mathcal{L}(A)$ é uma aplicação $q: M \rightarrow A$ satisfazendo as seguintes propriedades:

(i) $q(\lambda x)=\lambda^{2} q(x)$, para todo $x \in M, \lambda \in A$.

(ii) $b_{q}(x, y)=q(x+y)-q(x)-q(y)$ define em $M$ uma forma bilinear simétrica $b_{q}: M \times M \rightarrow A$.

O par $(M, q)$ é chamado um módulo quadrático sobre $A$ e $\left(M, b_{q}\right)$ seu módulo bilinear associado. Se $\left(M, b_{q}\right)$ é não singular diremọ que $(M, q)$ é não singular, ou é um espaço quadrático. Uma isometria entre dois módulos quadráticos $\left(M_{1}, q_{1}\right)$ e $\left(M_{2}, q_{2}\right)$ é um isomorfismo de $A$-módulos $\varphi: M_{1} \rightarrow M_{2}$, com a seguinte propriedade $q_{1}(x)=q_{2}(\varphi(x))$, para todo $x \in M_{1}$. Quando existe uma isometria entre $\left(M_{1}, q_{1}\right)$ e $\left(M_{2}, q_{2}\right)$ dizemos que os módulos quadráticos são isométricos e também denotamos por $\left(M_{1}, q_{1}\right) \simeq\left(M_{2}, q_{2}\right)$, ou simplesmente por $q_{1} \simeq q_{2}$, ou ainda $M_{1} \simeq M_{2}$. Naturalmente, neste caso, os módulos bilineares associados são também isométricos.

Observação 2.3 Se assumirmos que $2 \in A^{\star}$, ternos uma correspondência biunivoca entre os módulos bilineares e os módulos quadráticos sobre $A$. Esta correspondência associa 
ao módulo bilinear $(M, b)$ o módulo quadrático $\left(M, q_{b}\right)$, onde $q_{b}(x)=\frac{1}{2} b(x, x)$ para todo $x \in M$, e reciprocamente, o módulo quadrático $(M, q)$ corresponde ao módulo bilinear $\left(M, b_{q}\right)$. Verifica-se imediatamente que $q_{b_{q}}=q$ e $b_{q_{b}}=b$.

Se $(M, q)$ é um módulo quadrático sobre $A$ e $\left\{x_{1}, \ldots, x_{n}\right\}$ é uma base de $M$, então a forma quadrática $q$ é determinada pela matriz quadrada $\left(q_{i j}\right)$, onde $q_{i j}=b_{q}\left(x_{i}, x_{j}\right)$ se $i \neq j$ e $q_{i i}=q\left(x_{i}\right)$ se $i=j, 1 \leq i, j \leq n$, pois para $x=\sum_{i=1}^{n} \alpha_{i} x_{i}$ em $M$, temos $q(x)=\sum_{i, j=1}^{n} q_{i j} \alpha_{i} \alpha_{j}$. Reciprocamente, para cada matriz simétrica $n \times n,\left(q_{i j}\right)$, sobre $A$ obtemos uma forma quadrática sobre $M$ dada pela mesma fórmula descrita acima. Analogamente ao caso bilinear, o módulo quadrático $(M, q)$ é não singular se, e somente se, det $\left(q_{i j}\right)$ é uma unidade em A.

Exemplo 2.4 Se $M=A x \oplus A y$ e $q(x)=\alpha, q(y)=\beta, b_{q}(x, y)=1$, temos $\left(q_{i j}\right)=\left(\begin{array}{ll}\alpha & 1 \\ 1 & \beta\end{array}\right)$. A matriz de $\left(M, b_{q}\right)$ é $\left(\begin{array}{cc}2 \alpha & 1 \\ 1 & 2 \beta\end{array}\right)$, então $(M, q)$ é não singular se, e somente se $1-4 \alpha \beta \in A^{\star}$. Neste caso escreveremos $q=[\alpha, \beta]$ para denotar este espaço quadrático.

Um particular espaço quadrático desta forma será usado com uma certa freqüência no decorrer do trabalho que julgamos necessário citá-lo aqui.

De (1.6) temos que existe um número inteiro $h \geq 0$ tal que $(2 h-1)(4 h-1) \in A^{\star}$. Considere o módulo quadrático $\left(M^{\prime}, q^{\prime}\right)$, onde $M^{\prime}=A x \oplus A y$ e $q^{\prime}(x)=1, q^{\prime}(y)=h$ e $b_{q^{\prime}}(x, y)=1$. A matriz de $q^{\prime}$ é $\left(\begin{array}{ll}1 & 1 \\ 1 & h\end{array}\right)$ e de sua forma bilinear associada é $\left(\begin{array}{rr}2 & 1 \\ 1 & 2 h\end{array}\right)$. Como $1-4 h \in A^{\star}$, temos que $\left(M, q^{\prime}\right)$ é um espaço quadrático sobre $A$, com $q^{\prime}=[1, h]$.

Proposição 2.5 Seja $(M, q)$ um módulo quadrático sobre A. Então existe uma forma bilinear $b_{0}: M \times M \rightarrow A$ com $q(x)=b_{0}(x, x)$ e $b_{q}(x, y)=b_{0}(x, y)+b_{0}(y, x)$ para todo $x, y \in M$.

Dem.: Desde que $M$ é livre, podemos escrever $M=A x_{1} \oplus A x_{2} \oplus \cdots \oplus A x_{n}$, onde $\left\{x_{1}, \ldots, x_{n}\right\}$ é uma base de $M$. Sejam $a_{i i}=q\left(x_{i}\right)$ e $a_{i j}=b_{q}\left(x_{i}, x_{j}\right)$ para $i \neq j$. Então definimos $b_{0}: M \times M \rightarrow A$ por $b_{0}\left(x_{i}, x_{k}\right)=a_{i k}$ se $i \leq k$ e $b_{0}\left(x_{i}, x_{k}\right)=0$ se $i>k$. Facilmente, verifica-se que esta forma bilinear satisfaz o requerido.

Denotamos a categoria dos espaços bilineares sobre $\mathrm{A}$ por $\operatorname{Bil}(A)$, onde os morfismos desta categoria são as isometrias. Analogamente para as formas quadráticas, denotamos por 
para todo $x, y \in M, z \in E$ e $\alpha, \beta \in A^{\prime}$. Agora se $\left\{x_{1}, x_{2}, \ldots, x_{n}\right\}$ é uma base de $M$ sobre $A$, então $\left\{x_{1} \otimes 1, x_{2} \otimes 1, \ldots, x_{n} \otimes 1\right\}$ é uma base de $M \otimes A^{\prime}$ sobre $A^{\prime}$. Como $\left(b_{i j}\right)=\left(b\left(x_{i}, x_{j}\right)\right)$ é a matriz associada ao espaço bilinear $(M, b)$, então, em relação à base $\left\{x_{1} \otimes 1, \ldots, x_{n} \otimes 1\right\}$ de $M \otimes A^{\prime}$, o módulo $A^{\prime}$-bilinear $\left(M \otimes A^{\prime}, b \otimes A^{\prime}\right)$ tem como matriz associada $\left(\left(b \otimes A^{\prime}\right)_{i j}\right)=\left(\left(b \otimes A^{\prime}\right)\left(x_{i} \otimes 1, x_{j} \otimes 1\right)\right)=\left(\varphi\left(b\left(x_{i}, x_{j}\right)\right)\right)=\left(\varphi\left(b_{i j}\right)\right)$, o que mostra que $b \otimes A^{\prime} \in \operatorname{Bil}\left(A^{\prime}\right)$. Analogamente, mostra-se que $q \otimes A^{\prime} \in \operatorname{Quad}\left(A^{\prime}\right)$. Dizemos que os espaços $\left(M \otimes A^{\prime}, b \otimes A^{\prime}\right)$ e $\left(E \otimes A^{\prime}, q \otimes A^{\prime}\right)$, são obtidos por extensão dos escalares dos espaços $(M, b)$ e $(E, q)$. Como exemplos particulares de extensão de escalares, temos a localização em um ideal maximal e a redução módulo um ideal de $A$.

Localização - Para um ideal maximal m de $A$, seja $\varphi: A \rightarrow A_{\mathfrak{m}} \circ$ homomorfismo canónico $\varphi(\alpha)=\frac{\alpha}{1}$, para todo $\alpha \in A . \operatorname{Dados}(M, b) \in \operatorname{Bil}(A)$ e $(M, q) \in \operatorname{Quad}(A)$, temos que $M \otimes A_{\mathfrak{m}} \cong M_{\mathfrak{m}}$ e, $b_{\mathfrak{m}}=b \otimes A_{\mathfrak{m}} \dot{e}$ dada por $b_{\mathfrak{m}}\left(\frac{x}{\alpha}, \frac{y}{\beta}\right)=\frac{b(x, y)}{\alpha \beta}$, respectivamente $q_{\mathrm{m}}\left(\frac{x}{\alpha}\right)=\frac{q(x)}{\alpha}$, para todo $x, y \in M$ e $\alpha, \beta \in A-\mathfrak{m}$. Os espaços $\left(M_{\mathfrak{m}}, b_{\mathfrak{m}}\right)$ e $\left(M_{\mathrm{m}}, q_{\mathrm{m}}\right)$ são chamados as localizações em $\mathrm{m}$ de $(M, b)$ e $(M, q)$, respectivamente.

Redução - Para um ideal a de $A$ e $\varphi: A \rightarrow A / a$, a projeção canônica, temos as extensões de escalares para todo $(M, b)$ em $\operatorname{Bil}(A)$ e $(M, q)$ em $\operatorname{Quad}(A),(M(\mathfrak{a}), b(\mathfrak{a}))$ em $\operatorname{Bil}(A / \mathfrak{a})$ e $(M(\mathfrak{a}), q(\mathfrak{a}))$ em $\operatorname{Quad}(A / \mathfrak{a})$, onde $M(\mathfrak{a})=M \otimes(A / \mathfrak{a}) \cong M / \mathfrak{a} M$ e $b(\mathfrak{a})(\bar{x}, \bar{y})=$ $\varphi(b(x, y))=\overline{b(x, y)}$, respectivamente $q(\mathfrak{a})(\bar{x})=\varphi(q(x))=\overline{q(x)}$, para todo $\bar{x}, \bar{y} \in M / \mathfrak{a} M$. Os espaços $(M(\mathfrak{a}), b(\mathfrak{a}))$ e $(M(\mathfrak{a}), q(\mathfrak{a}))$ são chamados redução módulo a de $(M, b)$ e $(M, q)$, respectivamente.

Com esta noção de redução e localização para módulos bilineares e quadráticos temos:

Proposição 2.10 Seja $(M, b)$ um módulo bilinear sobre A. São equivalentes:

(i) $(M, b)$ é não singular;

(ii) $\left(M_{\mathrm{m}}, b_{\mathrm{m}}\right)$ é não singular, para todo $\mathrm{m} \in \operatorname{Spm}(A)$;

(iii) $(M(\mathrm{~m}), b(\mathrm{~m}))$ é não singular, para todo $\mathrm{m} \in \operatorname{Spm}(A)$.

Dem.: É suficiente observarmos que dada uma base $\left\{x_{1}, \ldots, x_{n}\right\}$ de $M$, temos que $\left\{\frac{x_{1}}{1}, \ldots, \frac{x_{n}}{1}\right\}$ é uma base de $M_{m}$ sobre $A_{m}$ e que $\left\{x_{1}+m M, \ldots, x_{n}+\mathrm{m} M\right\}$ é uma base de $M(\mathrm{~m})$ sobre $A / \mathrm{m}$. Logo a demonstração segue de (1.3) e da definição de espaço não singular. 
Temos também o resultado análogo para módulos quadráticos.

Proposição 2.11 Seja $(M, q)$ um módulo quadrático sobre A. Sáo equivalentes:

(i) $(M, q)$ é não singular;

(ii) $\left(M_{\mathfrak{m}}, q_{\mathfrak{m}}\right)$ é não singular, para todo $\mathfrak{m} \in \operatorname{Spm}(A)$;

(iii) $(M(\mathrm{~m}), q(\mathfrak{m}))$ é não singular, para lodo $\mathrm{m} \in S p m(A)$.

Dem.: Análoga à anterior.

Outra forma de redução que usaremos no decorrer do trabalho é quando $A=F_{1} \times \ldots \times F_{r}$ é um produto finito de corpos e, $(M, b)$ um módulo bilinear sobre $A$. Neste caso, para cada $i=1, \ldots, r$ a $i$-ésima projeção canônica $\pi_{i}: A \longrightarrow F_{i}$ induz uma redução $\pi_{i}^{*}(M, b)=\left(M_{i}, b_{i}\right)$, onde $b_{i}=\pi_{i} \circ b$. De (1.8) temos que $M \cong M_{1} \times \ldots \times M_{r}$ e, $\pi=\left(\pi_{1}, \ldots, \pi_{r}\right)$ induz a redução

$$
\pi^{*}(M, b)=\left(M_{1}, b_{1}\right) \times \ldots \times\left(M_{r}, b_{r}\right)
$$

onde $b=\pi^{*} \circ b=\left(\pi_{1} \circ b, \ldots, \pi_{r} \circ b\right)=\left(b_{1}, \ldots, b_{r}\right)$. Com estas notações temos:

Proposição 2.12 Se $A=F_{1} \times \ldots \times F_{r}$ e $(M, b)$ é um módulo bilinear sobre $A$ com $M \cong M_{1} \times \ldots \times M_{r}$, então $b=\left(b_{1}, \ldots, b_{r}\right)$ e $(M, b)$ é não singular se, e somente se $\left(M_{i}, b_{i}\right)$ é não singular, para cada $i=1, \ldots, n$.

Dem.: Basta observar que a adjunta de $b$ é $d_{b}=\left(d_{b_{1}}, \ldots, d_{b_{r}}\right)$ e, conseqüentemente, $d_{b}$ é um isomorfismo se, e somente se cada $d_{b_{i}}$ o é, para $i=1, \ldots, r$.

\subsection{Subespaços}

Seja $(M, b)$ um módulo bilinear, respectivamente $(M, q)$ um módulo quadrático sobre um anel $A$. Para cada subconjunto $U$ de $M$, o conjunto

$$
U^{\perp}=\{x \in M ; b(x, y)=0, \text { para todo } y \in U\}
$$


respectivamente

$$
U^{\perp}=\left\{x \in M ; b_{q}(x, y)=0, \text { para todo } y \in U\right\},
$$

é um submódulo de $M$, chamado o complemento ortogonal de $U$ em relação à $b$, respectivamente $q$. Dizemos que dois subconjuntos $U, V$ de $M$ são ortogonais se $U \subseteq V^{\perp}$ ou, equivalentemente, $V \subseteq U^{\perp}$. Com esta terminologia temos o seguinte lema de imediata verificação.

Lema 2.13 Sejam $M$ um módulo bilinear ou quadrático sobre $A$ e $U, V$ subconjuntos de M. Em ambos os casos temos:

(i) Se $V \subseteq U$, então $U^{\perp} \subseteq V^{\perp}$;

(ii) $U \subseteq U^{\perp \perp}$;

(iii) $U^{\perp}=U^{\perp \perp \perp}$.

Dem.: Imediata.

Seja $M$ um módulo bilinear ou quadrático sobre $A$. Um submódulo $U$ de $M$ é dito ser um subespaço de $(M, b)$, respectivamente $(M, q)$ se $U$ é um somando direto de $M$. Dizemos que $x$ é um elemento primitivo de $M$ se $A x$ é um subespaço de $M$.

Se $U$ e $V$ são submódulos de $M$ tais que $M=U \oplus V$ e $U \subseteq V^{\perp}$, dizemos que $M$ é a soma ortogonal de $U$ e $V$ e denotamos por $M=U \perp V$. Denotaremos por $\left(U,\left.b\right|_{U}\right)$, respectivamente $\left(U,\left.q\right|_{U}\right)$ a restrição da forma bilinear $b$, respectivamente quadrática $q$ ao subespaço $U$ de $M$. É imediato que se $M=U \perp V$, então $(M, b) \simeq\left(U,\left.b\right|_{U}\right) \perp\left(V,\left.b\right|_{V}\right)$, respectivamente $(M, q) \simeq\left(U,\left.q\right|_{U}\right) \perp\left(V,\left.q\right|_{V}\right)$.

Um subconjunto $U \subseteq M$ é dito ser totalmente isotrópico se $U \subseteq U^{\perp}$ e no caso quadrático temos adicionalmente que $q(x)=0$, para todo $x \in U$. Dizemos que um elemento $x \in M$ é isotrópico se $A x$ é um subespaço totalmente isotrópico de $M$, ou seja, se $x$ é um elemento primitivo de $M$ tal que $b(x, x)=0$, respectivamente $q(x)=0$. Um elemento $y \in M$ é dito ser anisotrópico se $A y$ é um subespaço de $M \operatorname{com} b(y, y) \in A^{\star}$, respectivamente $q(y) \in A^{\star}$. Dizemos também que o espaço bilinear ou quadrático $M$ é um espaço bilinear isotrópico, respectivamente espaço quadrático isotrópico se $M$ contém um elemento isotrópico. Se todos os elementos de $M$ são anisotrópicos dizemos que $M$ é um espaço bilinear anisotrópico, respectivamente espaço quadrático anisotrópico.

Os próximos dois resultados serão utilizados no capítulo final deste trabalho. 
Proposição 2.14 Seja $(M, b)$ um espaço bilinear sobre $A$ tal que b® $[1, h]$ é um espaço quadrático isotrópico, onde $[1, h]$ é a forma quadrárica descrita em (2.4). Então o espaço bilinear $6 b$ é isotrópico, onde $6 b=b \perp b \perp \ldots \perp b$, seis vezes.

Dem.: Seja $\{x, y\}$ uma base de $q=[1, h]$, tal quc $q(x)=1, q(y)=h$ e $b_{q}(x, y)=1$. Desde que $b \otimes[1, h]$ é isotrópico, temos que existe um elemento primitivo $z=u \otimes x+v \otimes y \mathrm{em}$ $M \otimes(A x \oplus A y)$, tal que

$$
\begin{aligned}
0 & =(b \otimes[1, h])(z, z) \\
& =(b \otimes q)(u \otimes x, u \otimes x)+(b \otimes q)(v \otimes y, v \otimes y)+\left(b \otimes b_{q}\right)(u \otimes x, v \otimes y) \\
& =b(u, u) q(x)+b(v, v) q(y)+b(u, v) b_{q}(x, y)=b(u, u)+h b(v, v)+b(u, v) .
\end{aligned}
$$

Temos então $b(u+v, u+v)+b(u, u)+(2 h-1) b(v, v)=2 b(u, u)+2 b(u, v)+2 h b(v, v)=0$. Desde que $2 h-1$ é um inteiro positivo que pode ser escrito como uma soma de 4 quadrados, da igualdade acima obtemos que $6 b$ é isotrópico.

Proposição 2.15 Seja $(M, q)$ um espaço quadrático sobre A tal que bq é isotrópico. Então $2 q$ é uma forma quadrática isotrópica.

Dem.: Desde que $b_{q}$ é isotrópico, temos que existe um elemento primitivo $x \in M$ tal que $b_{q}(x, x)=0$. Mas $b_{q}(x, x)=2 q(x)$, e o resultado segue.

Proposição 2.16 Seja $M$ um módulo bilinear ou quadrático sobre A.

(i) Se $M$ é não singular e $U \subseteq M$ é um subespaço, então $U^{\perp}$ é um subespaço de $M e$ $U=U^{\perp \perp}$.

(ii) Seja $U \subseteq M$ um submódulo tal que $\left(U,\left.b\right|_{U}\right)$, respectivamente $\left(U,\left.q\right|_{U}\right)$, é não singular. Então $U$ é um subespaço de $M$ e $M=U \perp U^{\perp}$.

Dem.: Faremos a demonstração somente para o caso bilinear, no caso quadrático a demonstração é análoga.

Seja $b$ a forma bilinear sobre $M$. Suponhamos que $(M, b)$ é não singular e que $U$ é um subespaço de $M$. Então existe um submódulo $V$ de $M$ tal que $M=U \oplus V$. Logo, $M^{*}=(U \oplus V)^{*} \cong U^{*} \oplus V^{*}$ e, temos a seqüência exata $M^{*} \longrightarrow U^{*} \longrightarrow 0$. Como $(M, b)$ 
é um espaço bilinear não singular, temos que a adjunta de $b, d_{b}: M \rightarrow M^{*}$ é um isomorfismo. Compondo este isomorfismo com a seqüência acima, obtemos a seqüência exata $M \longrightarrow U^{*} \longrightarrow 0$, onde o núcleo da composta $d: M \rightarrow U^{*}$ é exatamente $U^{\perp}$. Agora, $U^{*}$ é projetivo, pois é somando direto do $A$-módulo livre $M^{*}$. Portanto a seqüência exata curta

$$
0 \longrightarrow U^{\perp} \longrightarrow M \longrightarrow U^{\star} \longrightarrow 0
$$

cinde, ou seja, $M \cong U^{\perp} \oplus U^{*}$. Mostrando que $U^{\perp}$ é um subespaço de $M$.

Mostremos agora que $U^{\perp}=U^{\perp \perp}$. Para simplificar a notação, denotaremos também por $b$ as restrições da forma bilinear $b$ aos subespaços de $M$. $O$ isomorfismo $d: \frac{M}{U^{\perp}} \rightarrow U^{*}$ induz uma forma bilinear não singular $b: U \times \frac{M}{U^{\perp}} \rightarrow A$, para todo subespaço $U$ de $M$, onde $b(x, \bar{y})=d(\bar{y})(x)$ para todo $x \in U$ e $\bar{y} \in\left(M / U^{\perp}\right)$. Em particular, para o subespaço $U^{\perp}$ temos uma forma bilinear não singular $b: U^{\perp} \times \frac{M}{U^{\perp \perp}} \rightarrow A$. Por outro lado, temos o isomorfismo $M \cong\left(U^{\perp}\right)^{*} \oplus U$ que decorre dos isomorfismos

$$
M^{*} \cong\left(U^{\perp}\right)^{*} \oplus U^{* *}, \quad d: M \rightarrow M^{*} \quad \text { e } \quad U^{* *} \cong U .
$$

Assim obtemos um isomorfismo $d: \frac{M}{U} \rightarrow\left(U^{\perp}\right)^{*}$ que induz uma forma bilinear não singular $b: U^{\perp} \times \frac{M}{U} \rightarrow A$. Destas duas bilineares e do fato que $U \subseteq U^{\perp \perp}$, segue que $U=U^{\perp \perp}$. Pois se $U \varsubsetneqq U^{\perp \perp}$ então existe $y \in U^{\perp \perp}-U$ e, das duas formas bilineares obtidas, temos $\frac{M}{U} \simeq\left(U^{\perp}\right)^{*} \simeq \frac{\grave{M}}{U^{\perp \perp}}$. Como $y \in U^{\perp \perp}$ então para todo $x \in U^{\perp}, d(y)(x)=b(x, y)=0$. Por outro lado, como $y \notin U$, temos que $d(y) \neq 0$. Assim existe $z \in U^{\perp}$ tal que $d(y)(z) \neq 0$, ou seja, $b(z, y) \neq 0$, o que é uma contradição. Logo $U=U^{\perp \perp}$, mostrando $(i)$.

Seja agora, $\left(U,\left.b\right|_{U}\right)$ um subespaço não singular de $M$. Então $d_{b \mid U}: U \rightarrow U^{*}$ é um isomorfismo. No entanto, cada elemento $x \in M$ define um elemento $x^{*}$ em $U^{*}$ por $x^{*}(y)=b(x, y)=d_{\left.b\right|_{U}}(x)(y)$, para todo $y \in U$. Como $d_{\left.b\right|_{U}}: U \rightarrow U^{*}$ é sobrejetora, temos que existe $z \in U$ tal que $d(z)=d(x)$, ou seja, $b(z, y)=b(x, y)$, para todo $y \in U$. Portanto $b(x, y)-b(z, y)=0$, implicando em $b(x-z, y)=0$, para todo $y \in U$. Assim $x-z \in U^{\perp} \mathrm{e}$, como $x=z+(x-z)$, temos que $M=U+U^{\perp}$. De $d_{\left.b\right|_{U}}: U \rightarrow U^{*}$ ser injetora segue que $\{0\}=K \operatorname{er}\left(d_{\left.b\right|_{U}}\right)=K \operatorname{er}\left(d_{b}\right) \cap U=U^{\perp} \cap U$, mostrando que $M=U \oplus U^{\perp}$. Isto, juntamente com $U \subseteq U^{\perp \perp}$ implica no item (ii).

Corolário 2.17 Sejam $(M, b)$ um módulo bilinear sobre $A$ e a um ideal contido no radical de Jacobson, $\partial(A)$, de $A$. Se $(M(\mathfrak{a}), b(\mathfrak{a}))$ admile uma decomposição ortogonal 
$M(\mathfrak{a})=N(\mathfrak{a}) \perp W(\mathfrak{a})$, com $N(\mathfrak{a})$ livre sobre $A / \mathfrak{a}$ tal que $(N(\mathfrak{a}), b(\mathfrak{a}))$ é não singular, então existe uma decomposição ortogonal $M=N \perp W$ de $(M, b)$ com $N$ livre sobre $A,(N, b)$ não singular tal que $N(\mathfrak{a})=\frac{N}{\mathfrak{a} N}$ e $W(\mathfrak{a})=\frac{W}{\mathfrak{a} W}$.

Dem.: Desde que $(N(\mathfrak{a}), b(\mathfrak{a}))$ é um subespaço não singular de $(M(\mathfrak{a}), b(\mathfrak{a}))$ com $N(\mathfrak{a})$ livre sobre $A / \mathfrak{a}$, temos que existe uma base $\left\{\overline{x_{1}}, \ldots, \overline{x_{n}}\right\}$ de $N(\mathfrak{a})$ sobre $A / \mathfrak{a}$ tal que $\operatorname{det}\left(b(\mathfrak{a})\left(\overline{x_{i}}, \overline{x_{j}}\right)\right) \in(A / \mathfrak{a})^{\star}$. De (1.9) temos que $N=A x_{1}+\ldots+A x_{n}$ é um $A$-módulo livre com base $\left\{x_{i}, \ldots, x_{n}\right\}$ e $N(\mathfrak{a})=\frac{N}{\mathfrak{a} N}$. A matriz da forma bilinear $\left.b\right|_{N},\left(b\left(x_{i}, x_{j}\right)\right)$, é tal que $\overline{\operatorname{det}\left(b\left(x_{i}, x_{j}\right)\right)}=\operatorname{det}\left(\overline{b\left(x_{i}, x_{j}\right)}\right)=\operatorname{det}\left(b(\mathfrak{a})\left(\overline{x_{i}}, \overline{x_{j}}\right)\right) \in(A / \mathfrak{a})^{\star}$. Usando (1.2) e a definição (2.1) temos que $(N, b)$ é um subespaço não singular de $M$, e o resultado segue de (2.16).

Para finalizar esta seção, mostraremos que todo espaço bilinear sobre um anel semilocal admite uma decomposição como soma ortogonal de subespaços de dimensão $\leq 2$. Para isto, introduziremos algumas definições e resultados auxiliares.

No que segue, faremos uso das seguintes notações; $\bar{A}$ para o anel $A / \partial(A)$, onde $\partial(A)$ é o radical de Jacobson de $A$ e $(\bar{M}, \bar{b})$ para a redução módulo $\partial(A)$ do espaço bilinear $(M, b) \in \operatorname{Bil}(A)$.

Dado $(M, b) \in \mathcal{B} i l(A)$, consideremos o subconjunto $\{b(x, x) ; x \in M\}$ de $A$. Se o ideal gerado por este subconjunto é todo o anel $A$, diremos que o espaço bilinear $(M, b)$ é próprio. Caso contrário, dizemos que $(M, b)$ é um espaço bilinear impróprio.

Segue imediatamente desta definição e de (1.2) que:

Lema 2.18 Sejam $A$ um anel semilocal e $(M, b) \in \operatorname{Bil}(A)$. Então $(M, b)$ é um espaço bilinear próprio se, e somente se $(\bar{M}, \bar{b})$ é um espaço bilinear próprio sobre $\bar{A}$.

Dem.: Imediata.

Agora, se $A=F_{1} \times \ldots \times F_{r}$ é um produto finito de corpos e $(M, b) \in \mathcal{B} i l(A)$, de (2.12), temos que $(M, b) \cong\left(M_{1}, b_{1}\right) \times \ldots \times\left(M_{r}, b_{r}\right)$, com $\left(M_{i}, b_{i}\right) \in \mathcal{B} i l\left(F_{i}\right)$ e $b_{i}=\pi_{i} \circ b$, para cada $i=1, \ldots, r$. Neste caso temos

Lema 2.19 $O$ espaço bilinear $(M, b)$ é próprio se,e somente se $\left(M_{i}, b_{i}\right)$ é próprio, para cada $i=1, \ldots, r$. 
Dem.: Basta observarmos que se a é o ideal de $A$ gerado por $\{b(x, x) ; x \in M\}$ e, para cada $j=1, \ldots, r, a_{j}$ é o ideal de $F_{j}$, gerado por $\left\{b_{j}\left(x_{j}, x_{j}\right) ; x_{j} \in M_{j}\right\}$ então, $\mathfrak{a}=a_{1} \times \ldots \times a_{r}$. Portanto $\mathfrak{a}=A$ se, e somente se, $\mathfrak{a}_{j}=F_{j}$, para cada $j=1, \ldots, r$.

No caso de $A$ ser um anel semilocal, temos o seguinte teorema de decomposição para espaços bilineares:

Teorema 2.20 Seja $(M, b)$ um espaço bilinear sobre um anel semilocal $A$.

(i) Se $(M, b)$ é próprio, então $(M, b)$ é uma soma ortogonal de subespaços de dimensão 1 , isto é, $M$ admite uma base ortogonal com relação à forma bilinear $b$;

(ii) Se $(M, b)$ é impróprio, então $(M, b)$ é uma soma ortogonal de subespaços de dimensão 2 da forma $\left(\begin{array}{ll}\alpha & 1 \\ 1 & \beta\end{array}\right)$, com $\alpha, \beta \in A$ tais que $1-\alpha \beta \in A^{\star}$.

Dem.: Usando (2.18) e (2.19) é suficiente mostrarmos o teorema para $(\bar{M}, \bar{b})$ sobre $\bar{A}=A / \partial(A)$. Podemos então assumir que $\partial(A)=\{0\}$, ou seja, que $A \cong F_{1} \times \ldots \times F_{r}$ é um produto finito de corpos e, conseqüentemente $M \cong M_{1} \times \ldots \times M_{r}$ e $b=\left(b_{1}, \ldots, b_{r}\right)$. Também, usando indução sobre $r$, é suficiente mostrarmos o caso em que $r=2$.

Seja $(M, b)=\left(M_{1}, b_{1}\right) \times\left(M_{2}, b_{2}\right) \in \operatorname{Bil}(A)$, onde $A=F_{1} \times F_{2}$, com $F_{1}, F_{2}$ corpos. Faremos agora a demonstração por indução sobre $\operatorname{dim}(M) . \operatorname{Se} \operatorname{dim}(M)=1$, nada temos a demonstrar.

Se $\operatorname{dim}(M)>1$ e $(M, b)$ é próprio, então de (2.19) temos que $\left(M_{i}, b_{i}\right)$ é um espaço bilinear próprio sobre o corpo $F_{i}$, para cada $i=1,2$. Como o ideal gerado por $\left\{b_{i}\left(x_{i}, x_{i}\right) ; x_{i} \in M_{i}\right\}$ é $F_{i}$, para cada $i=1,2$, temos que existem $x_{1} \in M_{1}, x_{2} \in M_{2}$ tais que $b_{1}\left(x_{1}, x_{1}\right) \neq 0$ e $b_{2}\left(x_{2}, x_{2}\right) \neq 0$. Portanto, $x=\left(x_{1}, x_{2}\right) \in M$ é tal que $b(x, x)=$ $\left(b_{1}\left(x_{1}, x_{1}\right), b_{2}\left(x_{2}, x_{2}\right)\right) \in A^{\star}=F_{1}^{\star} \times F_{2}^{\star}$ e, conseqüentemente $(A x, b)$ é um subespaço não singular de dimensão 1 de $(M, b)$. De (2.16) temos que $(M, b)=(A x, b) \perp(U, b)$, com $U=(A x)^{\perp}$. Se $(U, b)$ é próprio, então por hipótese de indução $(U, b)$ é uma soma ortogonal de subespaços de dimensão 1 e, logo, $(M, b)$ também o é.

Se $(U, b)$ é impróprio, desde que $(U, b)=\left(U_{1}, b_{1}\right) \times\left(U_{2}, b_{2}\right)$, temos de (2.19) que $\left(U_{1}, b_{1}\right)$ é impróprio ou $\left(U_{2}, b_{2}\right)$ é impróprio. Então, há dois casos a considerarmos: 
Caso 1 - Se $\left(U_{i}, b_{i}\right)$ é impróprio para $i=1,2$. Neste caso, desde que $(U, b)$ é não singular, temos que $\left(U_{i}, b_{i}\right)$ são não singulares e, existem $y=\left(y_{1}, y_{2}\right), z=\left(z_{1}, z_{2}\right) \in U$ tais que $b_{i}\left(y_{i}, z_{i}\right) \neq 0$ em $F_{i}, i=1,2$. Conseqüentemente, $b(y, z) \in A^{\star}$. Tomando $u_{1}=x+y$ e $u_{2}=x+\lambda z, \operatorname{com} \lambda=-\frac{b(x, x)}{b(y, z)} \in A$, temos que $\left(A u_{1}+A u_{2}, b\right)$ é um subespaço não singular $\operatorname{de}(M, b)$, pois $\left(b\left(u_{i}, u_{j}\right)\right)=\left(\begin{array}{ll}b\left(u_{1}, u_{1}\right) & b\left(u_{1}, u_{2}\right) \\ b\left(u_{2}, u_{1}\right) & b\left(u_{2}, u_{2}\right)\end{array}\right)=\left(\begin{array}{cc}b(x, x) & 0 \\ 0 & b(x, x)\end{array}\right)$. Mais ainda, $\left\{u_{1}, u_{2}\right\}$ é uma base ortogonal deste subespaço. Assim, de (2.16)

$$
(M, b)=\left(A u_{1}, b\right) \perp\left(A u_{2}, b\right) \perp(N, b)
$$

com $\left(A u_{2}, b\right) \perp(N, b)$ próprio que, por hipótese de indução, admite uma base ortogonal. Completando esta base com $u_{1}$, obtemos uma base ortogonal de $(M, b)$.

Caso 2 - Se um dos espaços $\left(U_{i}, b_{i}\right)$ é próprio, $i=1,2$. Renomeando se necessário, podemos assumir que $\left(U_{1}, b_{1}\right)$ é impróprio e $\left(U_{2}, b_{2}\right)$ é próprio. Então, existem $y_{1}, z_{1} \in U_{1}, y_{1} \neq z_{1}$, e $y_{2} \in U_{2}$ tais que $b_{1}\left(y_{1}, z_{1}\right) \neq 0$ em $F_{1}$ e $b_{2}\left(y_{2}, y_{2}\right) \neq 0$ em $F_{2}$. Agora, os elementos $u_{1}=\left(x_{1}+y_{1}, x_{2}\right)$ e $u_{2}=\left(x_{1}+\lambda z_{1}, y_{2}\right)$ de $M \operatorname{com} \lambda=-\frac{b_{1}\left(x_{1}, x_{1}\right)}{b_{1}\left(y_{1}, z_{1}\right)}$ em $F_{1}^{\star}$, são tais que $\left(b\left(u_{i}, u_{j}\right)\right)=\left(\begin{array}{cc}\left(b_{1}\left(x_{1}, x_{1}\right), b_{2}\left(x_{2}, x_{2}\right)\right) & (0,0) \\ (0,0) & \left(b_{1}\left(x_{1}, x_{1}\right), b_{2}\left(y_{2}, y_{2}\right)\right)\end{array}\right)$, ou seja $\left\{u_{1}, u_{2}\right\}$ é uma base ortogonal de um subespaço não singular de $(M, b) \mathrm{e}$, como no Caso 1 , temos que $(M, b)$ admite uma base ortogonal, implicando em (i).

Consideremos agora $\operatorname{dim}(M)>1$ onde $(M, b)=\left(M_{1}, b_{1}\right) \times\left(M_{2}, b_{2}\right)$ é um espaço bilinear impróprio sobre $A=F_{1} \times F_{2}$. De (2.19) temos que $\left(M_{1}, b_{1}\right)$ é impróprio ou $\left(M_{2}, b_{2}\right)$ é impróprio. Novamente temos dois casos a considerar:

Caso 1 -Se $\left(M_{i}, b_{i}\right)$ é impróprio para $i=1,2$. Nestas condições, como no Caso 1 acima, existem $y, z \in M, y \neq z$, tais que $b(y, z) \in A^{\star}$. $O$ conjunto $\{y, z\}$ forma uma base de um subespaço não singular de $(M, b)$, pois a matriz da forma bilinear $b$ em relação à estes elementos é $\left(\begin{array}{ll}b(y, y) & b(y, z) \\ b(z, y) & b(z, z)\end{array}\right)=\left(\begin{array}{cc}0 & b(y, z) \\ b(y, z) & 0\end{array}\right)$ que tem determinante invertível em $A$. Assim, mostramos que $(M, b)$ admite um subespaço não singular de dimensão 2 . Trocando $z$ por $\lambda z, \operatorname{com} \lambda=\frac{1}{b(y, z)}$, temos que $(M, b)$ admite um subespaço não singular 
da forma $\left(\begin{array}{ll}\alpha & 1 \\ 1 & \beta\end{array}\right), \operatorname{com} 1-\alpha \beta=1 \in A^{\star}$.

Caso 2 - Se um dos espaços $\left(M_{i}, b_{i}\right),(i=1,2$. $)$, é próprio. Sem perda de generalidade, podemos supor que $\left(M_{1}, b_{1}\right)$ é impróprio e $\left(M_{2}, b_{2}\right)$ é próprio. Como no Caso 2 anterior, podemos obter $\left\{y_{1}, z_{1}\right\} \subseteq U_{1},\left\{y_{2}, z_{2}\right\} \subseteq U_{2}$ tais que $b_{1}\left(y_{1}, z_{1}\right) \neq 0, b_{1}\left(y_{1}, y_{1}\right)=b_{1}\left(z_{1}, z_{1}\right)=$ $0, \quad b_{2}\left(y_{2}, z_{2}\right)=0, \quad b_{2}\left(y_{2}, y_{2}\right) \neq 0$ e $b_{2}\left(z_{2}, z_{2}\right) \neq 0$. Tomando $u_{1}=\left(y_{1}, y_{2}+z_{2}\right)$ e $u_{2}=$ $\left(\lambda_{1} z_{1}, \lambda_{2} z_{2}\right)$ em $M$, onde $\lambda_{1}=\frac{1}{b_{1}\left(y_{1}, z_{1}\right)} \in F_{1}^{\star}$ e $\lambda_{2}=\frac{1}{b_{2}\left(z_{2}, z_{2}\right)} \in F_{2}^{\star}$, temos que $\left\{u_{1}, u_{2}\right\}$ é uma base de um subespaço de dimensão 2 de $(M, b)$ e, a matriz da forma bilinear restrita à este subespaço é $\left(b\left(u_{i}, u_{j}\right)\right)=\left(\begin{array}{ll}\alpha & 1 \\ 1 & \beta\end{array}\right)$ onde $\alpha=b\left(u_{1}, u_{1}\right)=\left(0, b_{2}\left(y_{2}, y_{2}\right)+b_{2}\left(z_{2}, z_{2}\right)\right) \in A$ e $\beta=b\left(u_{2}, u_{2}\right)=\left(0, \lambda_{2}^{2} b_{2}\left(z_{2}, z_{2}\right)\right) \in A$ são tais que $1-\alpha \beta=(1,1)-\left(0, \frac{b_{2}\left(y_{2}, y_{2}\right)}{b_{2}\left(z_{2}, z_{2}\right)}+1\right)=$ $\left(1,-\frac{b_{2}\left(y_{2}, y_{2}\right)}{b_{2}\left(z_{2}, z_{2}\right)}\right) \in A^{\star}$. Agora, o item (ii) do teorema segue de (2.16) e da hipótese de indução, pois todo subespaço não singular de um espaço bilinear impróprio é também impróprio.

Corolário 2.21 Todo espaço bilinear próprio sobre um anel semilocal $A$ é da forma $\left\langle\alpha_{1}, \ldots, \alpha_{n}\right\rangle, \operatorname{com} \alpha_{i} \in A^{\star}, 1 \leq i \leq n$.

Dem.: Imediata.

\subsection{Bases Relacionadas}

O principal objetivo desta seção é apresentar a noção de bases relacionadas para espaços bilineares sobre anéis semilocais, que generaliza a noção de cadeia-equivalência de Witt para espaços bilineares sobre corpos de característica distinta de 2. Os resultados aqui apresentados serão usados no próximo capítulo para o estudo da estrutura do anel de Witt bilinear.

Sejam $(M, b)$ um espaço bilinear sobre $A$ e $\mathcal{B}$ e $\mathcal{B}^{\prime}$ bases ortogonais de $(M, b)$. Dizemos que $\mathcal{B}$ e $\mathcal{B}^{\prime}$ estão $n$-relacionadas se existe uma seqüência finita $\mathcal{B}_{1}, \mathcal{B}_{2}, \ldots, \mathcal{B}_{k}$ de bases ortogonais de $(M, b) \operatorname{com} \mathcal{B}=\mathcal{B}_{1}, \mathcal{B}^{\prime}=\mathcal{B}_{k} \in \mathcal{B}_{i}$ diferindo de $\mathcal{B}_{i+\imath}$ em no máximo $n$ elementos. $O$ próximo resultado vale para um anel comutativo qualquer. 
Lema 2.22 Seja $(M, b) \in \mathcal{B i l}(A)$ com bases ortogonais $\mathcal{B}=\left\{x_{1}, \ldots, x_{m}\right\}$ e $\mathcal{B}^{\prime}=\left\{x_{1}^{\prime}, \ldots, x_{m}^{\prime}\right\} . S e \overline{x_{i}}=\overline{x_{i}}$ em $\bar{M}$, para todo $i=1, \ldots, m$, então $\mathcal{B}$ está 2 -relacionada $\operatorname{com} \mathcal{B}^{\prime}$.

Dem.: Usando indução sobre $m$, é suficiente mostrarmos que $\mathcal{B}$ está 2 -relacionada com uma base ortogonal $\left\{x_{1}^{\prime}, y_{2}, \ldots, y_{m}\right\}$, com $\overline{y_{i}}=\overline{x_{i}}$, para $i=2, \ldots, m$, pois $\left\{y_{2}, \ldots, y_{m}\right\}$ e $x_{2}^{\prime}, \ldots, x_{m}^{\prime}$ são bases ortogonais de $\left(A x_{1}^{\prime}\right)^{\perp}$.

Desde que $\overline{x_{1}}=\overline{x_{1}}$ renumerando, se necessário, podemos escrever $x_{1}^{\prime}=\left(1+\alpha_{1}\right) x_{1}+$ $\sum_{i=2}^{t} \alpha_{i} x_{i}$, com $\alpha_{i} \in \partial(A)$, não nulos para $2 \leq i \leq t$. Agora continuamos a demonstração usando indução sobre $t$. Se $x_{1}^{\prime}=\left(1+\alpha_{1}\right) x_{1}$, desde que, de (1.1) $1+\alpha_{i} \in A^{\star}$, temos que $\left\{x_{1}^{\prime}, x_{2}, \ldots, x_{m}\right\}$ é uma base ortogonal de $M, 1$-relacionada com $\mathcal{B}$ e, o lema esta mostrado neste caso.

Se $t \geq 2$, seja $x_{1}^{\prime \prime}=\left(1+\alpha_{1}\right) x_{1}+\alpha_{t} x_{t} \in M$. Temos então $b\left(x_{1}^{\prime \prime}, x_{1}^{\prime \prime}\right)=\left(1+\alpha_{1}\right)^{2} b\left(x_{1}, x_{1}\right)+$ $\left(\alpha_{t}\right)^{2} b\left(x_{t}, x_{t}\right)$, ou seja, $b\left(x_{1}^{\prime \prime}, x_{1}^{\prime \prime}\right) \equiv b\left(x_{1}, x_{1}\right) \bmod \partial(A)$, donde concluimos que $A x_{1}^{\prime \prime}$ é um subespaço não singular de $A x_{1} \perp A x_{t}$. Assim, de (2.16) obtemos que $A x_{1} \perp A x_{t} \simeq A x_{1}^{\prime \prime} \perp A x_{t}^{\prime \prime}$, onde $x_{t}^{\prime \prime}=x_{t}-\frac{b\left(x_{t}, x_{1}^{\prime \prime}\right)}{b\left(x_{1}^{\prime \prime}, x_{1}^{\prime \prime}\right)} x_{1}^{\prime \prime}$. Agora a base ortogonal de $M$

$$
\mathcal{B}^{\prime \prime}=\left\{x_{1}^{\prime \prime}, x_{2}, \ldots, x_{t-1}, x_{t}^{\prime \prime}, x_{t+1}, \ldots, x_{m}\right\}
$$

está claramente 2 -relacionada $\operatorname{com} \mathcal{B}$. Também $\overline{\mathcal{B}}=\overline{\mathcal{B}^{\prime \prime}}$, onde $\overline{\mathcal{B}}=\left\{\overline{x_{1}}, \ldots, \overline{x_{m}}\right\}$. Em relação à $\mathcal{B}^{\prime \prime}$ temos $x_{1}^{\prime}=x_{1}^{\prime \prime}+\sum_{i=2}^{t-1} \alpha_{i} x_{i}$ e, conseqüentemente, o resultado segue por indução sobre $t$.

Lema 2.23 Sejam $(M, b)$ um espaço bilinear sobre um corpo $A, \mathcal{B}=\left\{x_{1}, \ldots, x_{m}\right\}$ uma base ortogonal de $M$ e y um vetor anisotrópico de $M$. Então existe uma base ortogonal $\mathcal{B}^{\prime}=\left\{y, y_{2}, \ldots, y_{m}\right\}$ de $M$ que está 4 -relacionada $\operatorname{com} \mathcal{B}$.

Dem.: Seja $\alpha_{i}=b\left(x_{i}, x_{i}\right), i=1, \ldots, m$. Renumerando os índices se necessário, podernos supor que $y=\sum_{i=1}^{t} \lambda_{i} x_{i}$, com $\lambda_{i} \neq 0$ para todo $i=1, \ldots, t$.

Faremos a demonstração por indução sobre $t$. $O$ caso $t=1$ é obtido como na demonstração do lema anterior. Suponhamos então que $t>1$ e separemos em dois casos:

Caso 1 - Existem inteiros $i, j$, com $1 \leq i<j \leq t$ tais que $\left(\lambda_{i}\right)^{2} \alpha_{i}+\left(\lambda_{j}\right)^{2} \alpha_{j} \neq 0$. Neste 
caso, como no final da demonstração do lema anterior, existe $x_{j}^{\prime} \in M$ que juntamente com $x_{i}^{\prime}=\lambda_{i} x_{i}+\lambda_{j} x_{j}$, formam uma base ortogonal do subespaço $A x_{i} \perp A x_{j}$ de $M$. Então, a base ortogonal $\mathcal{B}_{1}=\left\{x_{1}, \ldots, x_{i-1}, x_{i}^{\prime}, \ldots, x_{j}^{\prime}, \ldots, x_{m}\right\}$ está 2 -relacionada com $\mathcal{B}$ e, desde que $y$ é uma combinação linear de $t-1$ elementos da base $\mathcal{B}_{1}$, a demonstração segue por hipótese de indução.

Caso 2 - Para todo $i, j$, com $1 \leq i<j<t$, temos $\left(\lambda_{i}\right)^{2} \alpha_{i}+\left(\lambda_{j}\right)^{2} \alpha_{j}=0$. Sejam $\alpha=$ $b(y, y) \neq 0$ e $\beta_{i}=\left(\lambda_{i}\right)^{2} \alpha_{i}$, para $1 \leq i \leq t$. Então, no corpo $A$ temos, $\beta_{i}+\beta_{j}=0$ para $i \neq j$ e $\sum_{i=1}^{t} \beta_{i}=\alpha \neq 0$. Isto implica que $t$ é ímpar e $\alpha=\beta_{i}$ para cada $i=1, \ldots, t$. Assim, para $i \neq j, 2 \alpha=\beta_{i}+\beta_{j}=0$, ou seja, $A$ é um corpo de caracteristica 2. Mais ainda, desde que $\left(\lambda_{i}\right)^{2} \alpha_{i}=\beta_{i} \neq 0$ para cada $i=1, \ldots, t$, fazendo uma série de mudanças de bases ortogonais

$$
\begin{gathered}
\mathcal{B}=\left\{x_{1}, \ldots, x_{m}\right\} \rightarrow\left\{\lambda_{1} x_{1}, x_{2}, \ldots, x_{m}\right\} \rightarrow\left\{\lambda_{1} x_{1}, \lambda_{2} x_{2}, \ldots, x_{m}\right\} \rightarrow \\
\cdots \rightarrow\left\{\lambda_{1} x_{1}, \lambda_{2} x_{2}, \ldots, \lambda_{t} x_{t}, x_{t+1}, \ldots, x_{m}\right\},
\end{gathered}
$$

sem perda de generalidade podemos supor que $y=\sum_{i=1}^{t} x_{i}$ com $t \geq 3$ impar e $\alpha_{i}=$ $b(y, y)=\alpha \neq 0$ para todo $i=1, \ldots, t$. Mostremos agora que nestas condições, temos $t<m=\operatorname{dim}(M)$. Desde que $y$ é anisotrópico e $m>1$, temos que existe $z \in(A y)^{\perp}$ tal que $b(z, z) \neq 0$. Seja $z=\sum_{i=1}^{m} \gamma_{i} x_{i}$. Então $0=b(y, z)=\left(\sum_{i=1}^{t} \gamma_{i}\right) \alpha$. Como $A$ tem característica 2 , temos que $0=(b(y, z))^{2}=\left(\sum_{i=1}^{t} \gamma_{i}^{2} \alpha\right) \alpha$, o que implica que $\sum_{i=1}^{t} \gamma_{i}^{2} \alpha=0$. Assim, se $t=m$, temos que $\sum_{i=1}^{t} \gamma_{i}^{2} \alpha=b(z, z)=0$, o que é uma contradição. Portanto $t<m$.

Se $A \neq \mathbb{F}_{2}$, então existe $\lambda \in A$ tal que, $\lambda \neq 0$ e $\beta=\alpha_{m} \lambda^{2}+\alpha \neq 0$. Tome

$$
x_{1}^{\prime}=\frac{\alpha}{\beta}\left(x_{1}+\lambda x_{m}\right) \text { e } x_{m}^{\prime}=\frac{\lambda}{\beta}\left(\lambda \alpha_{m} x_{1}+\alpha x_{m}\right) \text {. }
$$

É fácil verificar que $\left\{x_{1}^{\prime}, x_{m}^{\prime}\right\}$ é uma base ortogonal para $A x_{1} \perp A x_{m}, x_{1}=x_{1}^{\prime}+x_{m}^{\prime}$ e $b\left(x_{m}^{\prime}, x_{m}^{\prime}\right)=\frac{\lambda^{2} \alpha \alpha_{m}}{\beta} \neq \alpha$. A base $\mathcal{B}_{1}=\left\{x_{1}^{\prime}, x_{2}, \ldots, x_{m-1}, x_{m}^{\prime}\right\}$ está 2-relacionada com a base $\mathcal{B}$ e, em relação a base $\mathcal{B}_{1}$ temos $y=x_{1}^{\prime}+x_{2}+\ldots+x_{i}+x_{m}^{\prime}$, pois $x_{1}=x_{1}^{\prime}+x_{m}^{\prime}$. Esta expressão para $y$ é mais longa mas tem $b\left(x_{m}^{\prime}, x_{m}^{\prime}\right) \neq \alpha$. Assim, $b\left(x_{2}+x_{m}^{\prime}, x_{2}+x_{m}^{\prime}\right) \neq 0$ e, conseqüentemente, tomando $x_{2}^{\prime \prime}=x_{2}+x_{m}^{\prime}$, temos que existe um elemento $x_{m}^{\prime \prime} \in M$ tal que $\left\{x_{2}^{\prime \prime}, x_{m}^{\prime \prime}\right\}$ é uma base ortogonal para $A x_{2} \perp A x_{m}^{\prime}$. Nossa base $\mathcal{B}_{1}$ está 2 -relacionada com a base $\mathcal{B}_{2}=\left\{x_{1}^{\prime}, x_{2}^{\prime \prime}, x_{3}, \ldots, x_{m-1}, x_{m}^{\prime \prime}\right\}$ e, nesta base $y=x_{1}^{\prime}+x_{2}^{\prime \prime}+x_{3}+\ldots+x_{t}$. 
Agora, $b\left(x_{2}^{\prime \prime}+x_{3}, x_{2}^{\prime \prime}+x_{3}\right)=b\left(x_{2}+x_{m}^{\prime}+x_{3}, x_{2}+x_{m}^{\prime}+x_{3}\right)=b\left(x_{m}^{\prime}, x_{m}^{\prime}\right) \neq 0$. Para $x_{2}^{\prime \prime \prime}=x_{2}^{\prime \prime}+x_{3}$, existe um elemento $x_{3}^{\prime \prime \prime} \in M$ tal que $\left\{x_{2}^{\prime \prime \prime}, x_{3}^{\prime \prime \prime}\right\}$ é uma base ortogonal de $A x_{2}^{\prime \prime}+A x_{3}$. A base $\mathcal{B}_{2}$ está 2 -relacionada com a base $\mathcal{B}_{3}=\left\{x_{1}^{\prime}, x_{2}^{\prime \prime \prime}, x_{3}^{\prime \prime \prime}, x_{4}, \ldots, x_{m-1}, x_{m}^{\prime \prime}\right\}$ e, nesta base, $y$ é uma combinação linear de $t-1$ elementos e o resultado segue por hipótese de indução.

Finalmente, seja $A=\mathbb{F}_{2}$. Neste caso, $\alpha_{1}=\alpha_{2}=\ldots=\alpha_{m}=\alpha=1$. Considerando $M^{\prime}=A x_{1} \perp A x_{2} \perp A x_{3} \perp A x_{m}$ e $x^{\prime}=x_{1}+x_{2}+x_{3}$, temos $b\left(x^{\prime}, x^{\prime}\right)=1$ e, de (2.16), $M^{\prime}=A x^{\prime} \perp\left(A x^{\prime}\right)^{\perp}$. Agora temos $x_{m} \in\left(A x^{\prime}\right)^{\perp}$ e $\alpha_{m}=b\left(x_{m}, x_{m}\right)=1$, o que mostra que o espaço bilinear $\left(\left(A x^{\prime}\right)^{\perp}, b\right)$ é próprio e, de (2.20) admite uma base ortogonal $\left\{x_{2}^{\prime}, x_{3}^{\prime}, x_{m}^{\prime}\right\}$. Então a base ortogonal de $M, \mathcal{B}_{1}=\left\{x^{\prime}, x_{2}^{\prime}, x_{3}^{\prime}, x_{4}, \ldots, x_{m-1}, x_{m}^{\prime}\right\}$ está 4-relacionada com $\mathcal{B}$ e, com relação a esta base $y=x^{\prime}+x_{4}+\ldots+x_{t}$ (ou $y=x^{\prime}$ se $t=3$ ) e o resultado segue por hipótese de indução.

Observação 2.24 Note que, na demonstração do lema (2.23), mostramos que se $A \neq \mathbb{F}_{2}$, então existe uma base ortogonal de $M$, contendo $y$, que está 2 -relacionada com a base $\mathcal{B}$ e, se $A=\mathbb{F}_{2}$, existe uma base ortogonal de $M$, contendo $y$ que está 4 -relacionada com $\mathcal{B}$.

Mostremos agora o principal resultado desta seção.

Teorema 2.25 Sejam $(M, b)$ um espaço bilinear próprio sobre um anel semilocal $A, \mathcal{B}=$ $\left\{x_{1}, \ldots, x_{m}\right\}$ e $\mathcal{B}^{\prime}=\left\{y_{1}, \ldots, y_{m}\right\}$ bases ortogonais de $M$. Então $\mathcal{B}$ e $\mathcal{B}^{\prime}$ estão 4 -relacionadas. Mais ainda, se $|A / \mathrm{m}| \geq 3$ para todo $\mathrm{m} \in \operatorname{Spm}(A)$, então $\mathcal{B}$ e $\mathcal{B}^{\prime}$ estão 2-relacionadas.

Dem.: Reindexando os $x_{i}$ 's se necessário, podemos supor que $y_{1}=\sum_{i=1}^{t} \lambda_{i} x_{i}$, com $\lambda_{i} \neq 0$. Se $A$ é um corpo, então de (2.23) temos que $\mathcal{B}$ está 4-relacionada (2-relacionada se $A \neq \mathbb{F}_{2}$ ), com uma base ortogonal $\left\{y_{1}, z_{2}, \ldots, z_{m}\right\}$. Desde que $\left\{y_{2}, \ldots, y_{m}\right\}$ e $\left\{z_{2}, \ldots, z_{m}\right\}$ são bases ortogonais do espaço bilinear próprio $\left(A y_{1}\right)^{\perp}$, aplicando indução sobre $m$ temos o teorema neste caso.

Se $\mathcal{J}(A)=\{0\}$, então $A=F_{1} \times \cdots \times F_{r}, \operatorname{com} F_{i}$ corpo para $i=1, \ldots, r$. De $(2.19)$ temos que $(M, b)=\left(M_{1}, b_{1}\right) \times \cdots \times\left(M_{r}, b_{r}\right) \cong\left(M_{1} \times \cdots \times M_{r},\left(b_{1}, \ldots, b_{r}\right)\right) \operatorname{com}\left(M_{i}, b_{i}\right)$ espaços bilineares próprios sobre $F_{i}$. Para cada $i=1, \ldots, r$, seja $e_{i}=(0, \ldots, 1,0, \ldots, 0) \in M$, onde o elemento 1 está na $i$-ésima coordenada. Então $e_{i} \mathcal{B}=\left\{e_{i} x_{1}, e_{i} x_{2}, \ldots, e_{i} x_{m}\right\}$ e $e_{i} \mathcal{B}^{\prime}=\left\{e_{i} y_{1}, e_{i} y_{2}, \ldots, e_{i} y_{m}\right\}$ são bases ortogonais de $\left(M_{i}, b_{i}\right)$. De (2.23) temos que estas 
bases estão $n$-relacionadas, $\operatorname{com} n=4$ ou 2 . Usando as notações $\epsilon_{i} \mathcal{B}, \epsilon_{i} \mathcal{B}^{\prime}$, à partir das cadeias de bases $n$-relacionando $(n=4$ ou 2$), e_{i} \mathcal{B}$ com $e_{i} \mathcal{B}^{\prime}$, para cada $i$, obtemos uma cadeia finita de bases $n$-relacionando $\left(n=4\right.$ ou 2) $\mathcal{B}$ e $\mathcal{B}^{\prime}$ da forma

$$
\begin{gathered}
\mathcal{B}=e_{1} \mathcal{B}+e_{2} \mathcal{B}+\cdots+e_{r} \mathcal{B} \rightarrow \cdots \rightarrow e_{1} \mathcal{B}^{\prime}+e_{2} \mathcal{B}+\cdots+e_{r} \mathcal{B} \rightarrow \cdots \rightarrow \\
\cdots \rightarrow e_{1} \mathcal{B}^{\prime}+e_{2} \mathcal{B}^{\prime}+\cdots+e_{r} \mathcal{B} \rightarrow \cdots \rightarrow e_{1} \mathcal{B}^{\prime}+\cdots+e_{r} \mathcal{B}^{\prime}=\mathcal{B}^{\prime}
\end{gathered}
$$

o que mostra o teorema para o caso em que $\partial(A)=\{0\}$.

Finalmente, sejam $(\bar{M}, \bar{b}), \overline{\mathcal{B}}, \overline{\mathcal{B}^{\prime}}$ as reduções módulo $A / \partial(A)$ de $(M, b), \mathcal{B}$ e $\mathcal{B}^{\prime}$ respectivamente.

Então as bases ortogonais $\overline{\mathcal{B}}$ e $\overline{\mathcal{B}^{\prime}}$ de $\bar{M}$ estão $n$-relacionadas para $n=2$ ou 4 . Sejam $\overline{\mathcal{B}}_{i}$ e $\overline{\mathcal{B}}_{i+1}$ duas bases ortogonais de $M$ adjacentes na cadeia de base que relaciona $\overline{\mathcal{B}}$ à $\overline{\mathcal{B}^{\prime}}$. Seja $\overline{M_{0}}$ o subespaço de $\bar{M}$ gerado pelos elementos de $\overline{\mathcal{B}}_{i}$ que não estão em $\overline{\mathcal{B}}_{i+1}$. Assim $\overline{M_{0}}$ tem uma base ortogonal $\left\{\overline{u_{1}}, \ldots, \overline{u_{n}}\right\}$, com $n=2$ ou 4 e $\bar{M}=\overline{M_{0}} \perp \overline{M_{1}}$, onde $\overline{M_{1}}$ tem uma base ortogonal $\overline{\mathcal{B}}_{i} \cap \overline{\mathcal{B}}_{i+1}=\left\{\bar{w}_{i_{1}}, \ldots, \bar{w}_{i_{k}}\right\}$. De (2.17), temos que $M$ tem uma base ortogonal $\mathcal{B}_{i}=$ $\left\{u_{1}, \ldots, u_{n}, w_{i_{1}}, \ldots, w_{i_{k}}\right\}$ que é um levantamento das respectivas bases de $\overline{M_{0}}$ e $\overline{M_{1}}$. Agora, os elementos de $\overline{\mathcal{B}}_{i+1}$ que não estão em $\overline{\mathcal{B}}_{i}$ geram $\left(\overline{M_{1}}\right)^{\perp} \simeq \overline{M_{0}}$. Seja então $\left\{\bar{v}_{1}, \ldots, \bar{v}_{n}\right\}$ a base de $\bar{M}_{0}$ contida em $\overline{\mathcal{B}}_{i+1}$. Novamente de (2.17) podemos encontrar um levantamento da base $\overline{\mathcal{B}}_{i+1}$ para uma base ortogonal de $M$ da forma $\left\{v_{1}, \ldots, v_{n}, w_{i_{1}}, \ldots, w_{i_{k}}\right\}$. Observe que $\mathcal{B}_{i}$ e $\mathcal{B}_{i+1}$ são bases de $M$ que diferem em $n$ elementos. Com este raciocínio, mostramos que $\mathcal{B}$ está $n$-relacionada $\left(n=2\right.$ ou 4) com uma base ortogonal $\mathcal{B}^{\prime \prime}=\left\{y_{1}^{\prime \prime}, y_{2}^{\prime \prime}, \ldots, y_{n}^{\prime \prime}\right\}$ tal que $y_{i}^{\prime \prime} \equiv y_{i} \bmod \mathcal{J}(A)$, ou seja, $\overline{\mathcal{B}^{\prime \prime}}=\overline{\mathcal{B}^{\prime}}$ e, agora o resultado segue de (2.22).

\subsection{Espaços Metabólicos e Hiperbólicos}

Nesta seção daremos a definição e a caracterização dos espaços metabólicos e hiperbólicos, os quais são essenciais para a definição dos anéis de Witt bilinear e quadrático que definiremos no próximo capítulo.

Seja $(U, b)$ um módulo bilinear sobre $A$, respectivamente $P \in \mathcal{L}(A)$. Definimos em $U \oplus U^{*}$ uma forma bilinear simétrica $b_{U}$ por

$$
b_{U}\left(u+u^{*}, v+v^{*}\right)=b(u, v)+u^{*}(v)+v^{*}(u)
$$


para todo $u, v \in U$ e $u^{*}, v^{*} \in U^{*}$. Respectivamente, definimos em $P \oplus P^{*}$ uma forma quadrática $q_{P}$ por

$$
q_{P}\left(x+x^{*}\right)=x^{*}(x)
$$

para todo $x \in P, x^{*} \in P^{*}$. Ao módulo bilinear $\left(U \oplus U^{*}, b_{U}\right)$ damos o nome de espaço metabólico e denotamos por $\mathbb{M}(U, b)$, ou simplesmente por $\mathbb{M}(U)$, ou ainda $\mathbb{M}(b)$. Respectivamente, ao módulo quadrático $P \oplus P^{*}$ damos o nome de espaço hiperbólico e denotamos por $\mathrm{H}[P]$. Mostremos a seguir que estes módulos são de fato não singulares.

Proposição 2.26 Sejam $(U, b)$ um módulo bilinear sobre $A$ e $P \in \mathcal{L}(A)$. Então $\mathbb{M}(U) \in$ $\operatorname{Bil}(A)$ e $\mathbb{H}[P] \in \operatorname{Quad}(A)$.

Dem.: Consideremos $\left\{e_{1}, \ldots, e_{n}, e_{1}^{*}, \ldots, e_{n}^{*}\right\}$ uma base $\operatorname{de} \operatorname{IM}(U)=U \oplus U^{*}$, onde $\left\{e_{1}, \ldots, e_{n}\right\}$ é uma base de $U$ e $\left\{e_{1}^{*}, \ldots, e_{n}^{*}\right\}$ é a base dual de $U^{*}$. A matriz de $b_{U}$ com relação a esta base é a matriz em blocos

$$
\left(b_{U}\left(e_{i}, e_{j}^{*}\right)\right)=\left(\begin{array}{cc}
B & I \\
I & 0
\end{array}\right)
$$

onde o bloco $B$ é a matriz associada ao módulo bilinear $(U, b), I$ é a matriz identidade $n \times n$ e 0 é a matriz nula $n \times n$. O determinante desta matriz é $\operatorname{det}(I) \operatorname{det}(I)$, isto é, a matriz associada a forma bilinear simétrica $b_{U}$ é invertível e, portanto $\mathbb{M}(U)$ é um espaço bilinear não singular. Mostra-se de maneira análoga que $\mathbb{H}[P] \in Q u a d(A)$.

Em $\mathbb{H}[P]$ existem dois subespaços totalmente isotrópicos, $P$ e $P^{*}$, com $P=P^{\perp}$ e $P^{*}=P^{* \perp}$. No caso de $\mathbb{M}(U)$ o subespaço $U^{*}$ é sempre totalmente isotrópico, o mesmo pode não ocorrer com $U$. Se $b=0$, então $U$ também é um subespaço totalmente isotrópico de $\mathbb{M}(U)$. Neste caso, dizemos que $\mathbb{M}(U, 0)$ é um espaço bilinear hiperbólico que também denotaremos por $\mathrm{H}(U)$.

Se $U=A x$, então $\mathbb{H}(U)=\left(\begin{array}{ll}0 & 1 \\ 1 & 0\end{array}\right)$, será chamado de plano hiperbólico e denotado simplesmente por $\mathbb{H}$. Todo espaço bilinear hiperbólico é uma soma ortogonal de planos hiperbólicos. De fato, consideremos $(U, 0)$ tal que $\operatorname{dim}(U)=n$. Sejam $\left\{e_{1}, \cdots, e_{n}\right\}$ e $\left\{e_{1}^{*}, \cdots, e_{n}^{*}\right\}$ bases de $U$ e $U^{*}$, respectivamente. Podemos considerar $\left\{e_{1}, e_{1}^{*}, e_{2}, e_{2}^{*}, \ldots, e_{n}, e_{n}^{*}\right\}$ como base de $U \oplus U^{*}$ e em relação a esta base

$$
\mathrm{H}(U) \simeq\left(\begin{array}{ll}
0 & 1 \\
1 & 0
\end{array}\right) \perp \ldots \perp\left(\begin{array}{ll}
0 & 1 \\
1 & 0
\end{array}\right)
$$


com $n$ parcelas. Assim, $\mathbb{H}(U) \simeq n \mathbb{H}$, onde $n=\operatorname{dim}(U)$.

Consideremos agora, $(U, b)=(A x,\langle\alpha\rangle)$, então

$$
\mathbb{M}(\langle\alpha\rangle)=\left(\begin{array}{cc}
\alpha & 1 \\
1 & 0
\end{array}\right)
$$

Mais geralmente,

$$
\mathbb{M}\left(\left\langle\alpha_{1}, \ldots, \alpha_{n}\right\rangle\right) \simeq\left(\begin{array}{cc}
\alpha_{1} & 1 \\
1 & 0
\end{array}\right) \perp \ldots \perp\left(\begin{array}{cc}
\alpha_{n} & 1 \\
1 & 0
\end{array}\right)
$$

Teorema 2.27 Seja $M$ um espaço bilinear ou quadrático sobre $A$. Se $U \subseteq M$ é um subespaço totalmente isotrópico de $M$, então existe um subespaço $V \subseteq M$ tal que $M=$ $U^{\perp} \oplus V$ e $U \oplus V$ é um subespaço não singular. Além disso, no caso bilinear $U \oplus V e ́$ metabólico e no caso quadrático, $V$ também é totalmente isotrópico com $U \cong V^{*}$.

Dem.: Na demonstração $b$ denotará a forma bilinear sobre $M$ no caso bilinear, ou a forma bilinear associada a forma quadrática sobre $M$, no caso quadrático.

De (2.16) temos que $U^{\perp}$ é um subespaço de $M$, então existe $V \subseteq M$ tal que $M=U^{\perp} \oplus V$. Na demonstração de (2.16), foi mostrado que $b: U \times \frac{M}{U^{\perp}} \longrightarrow A$ é não singular, isto é, $b: U \times V \rightarrow A$ é não singular. Logo $d: U \rightarrow V^{*}, x \mapsto b(x, \quad)$ e $d: V \longrightarrow U^{*}, y \mapsto b(, y)$ são isomorfismos. Em particular segue de $U \subseteq U^{\perp}$ e $U^{\perp} \cap V=\{0\}$ que $U \cap V=\{0\}$. Assim $U \oplus V$ é um subespaço de $M$. Mostremos que $b:(U \oplus V) \times(U \oplus V) \rightarrow A$ é não singular. Como $U \subseteq U^{\perp}$, a matriz de $b$ com relação a decomposição $(U \oplus V) \times(U \oplus V)=(U \times U) \oplus(U \times V) \oplus(V \times U) \oplus(V \times V)$, é a matriz em blocos

$$
B=\left(\begin{array}{cc}
0 & B_{12} \\
B_{12} & B_{22}
\end{array}\right)
$$

onde $B_{12}$ é a matriz de $\left.b\right|_{U \times V}$ e $B_{22}$ é a matriz de $\left.b\right|_{V \times V}$. Uma vez que $b: U \times V \longrightarrow A$ é não singular então $B_{12}$ é invertível. Assim

$$
B^{-1}=\left(\begin{array}{cc}
-B_{12}^{-1} B_{22} B_{12}^{-1} & B_{12}^{-1} \\
B_{12}^{-1} & 0
\end{array}\right)
$$

é a inversa de $B$, donde concluimos que $B$ é invertível, ou seja, $(U \oplus V, b)$ é não singular e de (2.16) temos que $M=(U \oplus V) \perp(U \oplus V)^{\perp}$, o que mostra a primeira parte do teorema. 
No caso em que $M$ é um espaço bilinear, considere $f: U \oplus V \rightarrow \mathbb{M}(V)=V \oplus V^{*}$ definida por $f(u+v)=v+d(u)$, para todo $u \in U$ e $v \in V$. Temos que $f$ é um isomorfismo, pois é a soma da identidade com o isomorfismo $d$. Mostremos que de fato $f$ é uma isometria; para todo $u+v, u^{\prime}+v^{\prime}$ em $U \oplus V$, temos

$$
\begin{gathered}
b_{V}\left(f(u+v), f\left(u^{\prime}+v^{\prime}\right)\right)=b_{V}\left(v+d(u), v^{\prime}+d\left(u^{\prime}\right)\right)=b\left(v, v^{\prime}\right)+d(u)\left(v^{\prime}\right)+d\left(u^{\prime}\right)(v)= \\
=b\left(v, v^{\prime}\right)+b\left(u, v^{\prime}\right)+b\left(u^{\prime}, v\right)=b\left(u+v, v^{\prime}\right)+b\left(u^{\prime}, v+u\right)=b\left(u+v, u^{\prime}+v^{\prime}\right) . \text { Portanto }
\end{gathered}
$$
$U \oplus V \simeq \mathbb{M}(V)$, completando a demonstração do teorema para espaços bilineares.

No caso em que $M$ é um espaço quadrático, se $V$ é totalmente isotrópico, nada mais temos a demonstrar. Se não, trocaremos $V$ por um espaço totalmente isotrópico $V^{\prime} \subseteq M$ com as requeridas propriedades. Para tanto, escolhemos uma forma bilinear $b_{0}: M \times M \rightarrow A$ com $q(x)=b_{0}(x, x)$ para todo $x \in E$, cuja existência é garantida por (2.5). Cada $y \in V$ define um elemento em $V^{*}$ por $z \mapsto b_{0}(y, z)$ para todo $z \in V$. Do isomorfismo $d: U \rightarrow V^{*}$ obtemos um único $u_{y} \in U$ satisfazendo $d\left(u_{y}\right)(z)=b_{0}(y, z)$ para todo $z \in V$, isto é, $b\left(u_{y}, z\right)=b_{0}(y, z)$. A unicidade de $u_{y}$ implica $u_{y+y^{\prime}}=u_{y}+u_{y^{\prime}} \mathrm{e}$ $u_{\lambda y}=\lambda u_{y}$ para todo $y, y^{\prime} \in V, \lambda \in A$. Então $V^{\prime}=\left\{y-u_{y} ; y \in V\right\}$ é um submódulo de $M$ e $V \cong V^{\prime}$ onde $y \mapsto y-u_{y}$ é o isomorfismo. Mostremos agora que $U \oplus V^{\prime}$ é um subespaço não singular de $M$. Com efeito, a partir dos isomorfismos obtidos em (2.16) e de $V \cong V^{\prime}$ acima temos

$$
\left(U \oplus V^{\prime}\right)^{*} \cong(U \oplus V)^{*} \cong U^{*} \oplus V^{*} \simeq V \oplus U \cong U \oplus V^{\prime},
$$

ou seja $U \oplus V^{\prime}$ é isomorfo ao seu dual onde os isomorfismos são induzidos pela adjunta de $b$ e/ou suas restrições, o que implica que este espaço é não singular.

Finalmente, mostremos que $V^{\prime}$ é totalmente isotrópico. De fato, desde que para cada $y \in V$ temos que $q\left(y-u_{y}\right)=q(y)-b\left(y, u_{y}\right)=q(y)-b_{0}(y, y)=0$, implicando no resultado.

Corolário 2.28 Se $x \in(M, b)$ é primitivo e isotrópico então existe $y \in M$ tal que $(A x \oplus A y, b)=\left(\begin{array}{cc}0 & 1 \\ 1 & b(y, y)\end{array}\right)$ é um subespaço metabólico não singular de $(M, b)$.

Dem.: No teorema anterior, consideremos $U=A x$, temos que existe $V=A y^{\prime}$ tal que $\left(A x \oplus A y^{\prime}, b\right)$ é não singular e metabólico. A matriz de $b$ com relação a base $\left\{x, y^{\prime}\right\}$ é dada 
por $\left(\begin{array}{cc}0 & b\left(x, y^{\prime}\right) \\ b\left(x, y^{\prime}\right) & b\left(y^{\prime}, y^{\prime}\right)\end{array}\right)$. Como $b$ é não singular, temos que $b\left(x, y^{\prime}\right) \in A^{*}$. Tomando $y=\frac{1}{b\left(x, y^{\prime}\right)} y^{\prime}$, obtemos que a matriz de $b$ com relação a base $\{x, y\}$ é $\left(\begin{array}{cc}0 & 1 \\ 1 & b(y, y)\end{array}\right)$, como queríamos.

O próximo resultado caracteriza os espaços metabólicos e hiperbólicos, além de ser fundamental para a definição dos anéis de Witt.

Teorema 2.29 Seja $M$ um espaço quadrático ou bilinear sobre A. Então $M$ é um espaço hiperbólico, respectivamente metabólico se, e somente se $M$ contém um subespaço $U$ totalmente isotrópico maximal, isto é, $U=U^{\perp}$. Mais ainda, no caso quadrático $M \simeq \mathbb{H}[U]$.

Dem.: Se $M$ é hiperbólico, é imediato que $M$ possui um subespaço isotrópico maximal. Agora seja $(M, b)$ um espaço metabólico, então $M=V \oplus V^{*}$ para algum módulo bilinear $\left(V, b^{\prime}\right)$. Como $V^{*}$ é um subespaço totalmente isotrópico, temos que $V^{*} \subseteq\left(V^{*}\right)^{\perp}$. Mostraremos que, na verdade $V^{*}=\left(V^{*}\right)^{\perp}$. Consideremos $\left\{x_{1}, \ldots, x_{n}\right\}$ uma base de $V$ e $\left\{x_{1}^{*}, \ldots, x_{n}^{*}\right\}$ a base dual de $V^{*}$. Para $v+v^{*} \in\left(V^{*}\right)^{\perp}$, temos $b_{V}\left(v+v^{*}, v_{1}^{*}\right)=0$, para todo $v_{1}^{*} \in V^{*}$, em particular, $b_{V}\left(v+v^{*}, x_{i}^{*}\right)=0$, para todo $i=1, \ldots, n$. Mas $b_{V}\left(v+v^{*}, x_{i}^{*}\right)=b(v, 0)+v^{*}(0)+x_{i}^{*}(v)=0$, ou seja, $x_{i}^{*}(v)=0$, para todo $i=1, \ldots, n$. Escrevendo $v=u_{1} x_{1}+\ldots+u_{n} x_{n}$, temos $0=x_{i}^{*}(v)=x_{i}^{*}\left(u_{1} x_{1}+\ldots+u_{n} x_{n}\right)=u_{i}$, para todo $i=1, \ldots, n$. Logo $v=0$ e, assim $v+v^{*}=v^{*}$ está em $V^{*}$, ou seja, $\left(V^{*}\right)^{\perp} \subseteq V^{*}$. Portanto $V^{*}=\left(V^{*}\right)^{\perp}$.

Reciprocamente se $M$ é bilinear e $U \subseteq M$ é um subespaço tal que $U=U^{\perp}$, por (2.27) $M=U^{\perp} \oplus V=U \oplus V$ e $(U \oplus V, b)$ é metabólico. Agora se $M$ é quadrático e $U$ é como acima satizfazendo $q(x)=0$, para todo $x \in U$, então da demonstração de (2.16) item (i), temos que $M \cong U^{\perp} \oplus U^{*}$. Tomando $V=U^{*}$ totalmente isotrópico conforme (2.27) temos $V^{*}=U^{* *} \cong U \mathrm{e}$, como $U=U^{\perp}$, obtemos $M \cong U \oplus V \cong U \oplus U^{*}=\mathbb{H}[U]$. Da demonstração de (2.27) $d_{q}: V \rightarrow V^{*}, x \mapsto b_{q}(x, \quad)$ é um isomorfismo. Definindo $f: M=U \oplus V \rightarrow \mathbb{H}[U]=U \oplus U^{*}$ por $f(u+v)=u+d_{q}(v)$, para $u \in U, v \in V$, temos que $f$ é claramente um isomorfismo. Resta mostrarmos que este isomorfismo é de fato uma isometria. Com efeito, $q_{U}(f(u+v))=q_{U}\left(u+d_{q}(v)\right)=d_{q}(v)(u)=b_{q}(u, v)=q(u+v)$. Assim, obtemos $M \simeq \mathbb{H}[U]$. 
Corolário 2.30 Para $P \in \mathcal{L}(A),(E, q) \in \operatorname{Quad}(A),(M, b) \in \mathcal{B} i l(A) \in\left(U, b^{\prime}\right)$ um módulo bilinear sobre A, temos:

(i) $(E, q) \perp(E,-q) \simeq \mathbb{H}[E]$;

(ii) $(M, b) \perp(M,-b) \simeq \mathbb{M}(U)$;

(iii) $\mathrm{M}(U) \otimes(E, q) \simeq \mathbb{H}[U \otimes E]$;

(iv) $(M, b) \otimes \mathbb{H}[P] \simeq \mathbb{H}[M \otimes P]$;

(v) $\mathbb{H}[P] \odot(E, q) \simeq \mathbb{H}[P \otimes E]$;

(vi) $\mathrm{M}(U) \otimes(M, b) \simeq \mathrm{M}[U \otimes M]$.

Dem.: Para mostrarmos os itens $(i)$ e (ii) basta usar o teorema anterior e o fato que $V=\{(x, x) ; x \in E\}$ e $V^{\prime}=\{(x, x) ; x \in U\}$ são, respectivamente, subespaços totalmente isotrópicos maximais de $(E, q) \perp(E,-q)$ e $(U, b) \perp(U,-b)$.

Para os itens restantes, mostraremos apenas o item (iii), os demais são demonstrados de maneira similar. Denotaremos também por $q$ a forma quadrática definida em $\mathrm{M}(U) \otimes E$. Observemos que o espaço quadrático $\mathrm{M}(U) \otimes E$ contém o subespaço totalmente isotrópico maximal $U^{*} \otimes E$. De fato, para qualquer $u^{*} \in U^{*}, x \in E$ temos:

$$
q\left(u^{*} \otimes x\right)=b_{U}\left(u^{*}, u^{*}\right) q(x)=0
$$

ou seja, $\left(U^{*} \otimes E\right) \subseteq\left(U^{*} \otimes E\right)^{\perp}$. Para mostrarmos a outra inclusão, basta considerarmos os elementos da forma $\left(\left(u_{i}+u_{j}^{*}\right) \otimes x_{k}\right), u_{i}$ em alguma base de $U, u_{j}^{*}$ numa base de $U^{*}$ e $x_{k}$ numa base de $E$, que estão em $\left(U^{*} \otimes E\right)^{\perp}$, pois estes elementos geram $\operatorname{M}(U) \otimes E$. Para tais elementos temos para todo $u^{*} \in U^{*}$ e $x \in E$

$$
0=b_{b_{U} \otimes q}\left(\left(u_{i}+u_{j}^{*}\right) \otimes x_{k}, u^{*} \otimes x\right)=\left(b_{U} \otimes b_{q}\right)\left(\left(u_{i}+u_{j}^{*}\right) \otimes x_{k}, u^{*} \otimes x\right) .
$$

Como $x_{k}$ pertence a alguma base de $E$ e $b_{q}$ é não singular, temos que existe $x^{\prime} \in E$ tal que $b_{q}\left(x_{k}, x^{\prime}\right) \in A^{\star}$. Logo

$$
0=\left(b_{U} \otimes b_{q}\right)\left(\left(u_{i}+u_{j}^{*}\right) \otimes x_{k}, u^{*} \otimes x^{\prime}\right)=b_{U}\left(u_{i}+u_{j}^{*}, u^{*}\right) b_{q}\left(x_{k}, x^{\prime}\right)
$$

implica que $b_{U}\left(u_{i}+u_{j}^{*}, u^{*}\right)=u^{*}\left(u_{i}\right)=0$, para todo $u^{*} \in U^{*}$, isto é, $u_{i}=0$, para todo $u_{i}$ em uma base de $U$. Assim, $\left(U^{*} \otimes E\right)^{\perp}=U^{*} \otimes E$. Com isso temos que $\mathrm{IM}(U) \otimes E$ é isométrico a 
$\mathbb{H}\left[U^{*} \otimes E\right]=\left(U^{*} \otimes E\right) \oplus\left(U^{*} \otimes E\right)^{*} \cong\left(U^{*} \otimes E^{*}\right) \oplus(U \otimes E) \simeq \mathbb{H}[U \otimes E]$, desde que $U^{* *} \cong U$ e $E^{*} \cong E$.

Proposição 2.31 Seja $(U, b)$ um módulo bilinear sobre A. Então:

$$
\mathbb{M}(U, b) \perp \mathbb{M}(U,-b) \simeq \mathbb{H}(U) \perp \mathbb{M}(U,-b) .
$$

Dem.: Seja $\left\{x_{1}^{*}, \ldots, x_{n}^{*}, x_{1}, \ldots, x_{n}\right\}$ uma base de $U^{*} \oplus U$, onde $\left\{x_{1}, \ldots, x_{n}\right\}$ é uma base de $U$ e $\left\{x_{1}^{*}, \ldots, x_{n}^{*}\right\}$ é a base dual de $U^{*}$. Com relação a esta base temos que

$$
\mathbb{M}(U, b)=\left(\begin{array}{ll}
0 & I \\
I & B
\end{array}\right),
$$

onde $I$ é a matriz identidade $n \times n, 0$ é a matriz nula $n \times n$ e $B$ é a matriz de $(U, b)$ em relação à base dada. Note que em relação a esta mesma base

$$
\mathbb{M}(U,-b)=\left(\begin{array}{cc}
0 & -I \\
-I & -B
\end{array}\right),
$$

pois, $-b_{U}(x, y)=-b(x, y)-u^{*}(v)-v^{*}(u)$.

Considerando a matriz em blocos invertível $C=\left(\begin{array}{cccc}I & 0 & I & B \\ 0 & I & 0 & 0 \\ 0 & 0 & I & 0 \\ 0 & I & 0 & I\end{array}\right)$, obtemos

$$
\left(\begin{array}{cccc}
I & 0 & 0 & 0 \\
0 & I & 0 & I \\
I & 0 & I & 0 \\
B & 0 & 0 & I
\end{array}\right)\left(\begin{array}{cccc}
0 & I & 0 & 0 \\
I & B & 0 & 0 \\
0 & 0 & 0 & -I \\
0 & 0 & -I & -B
\end{array}\right)\left(\begin{array}{cccc}
I & 0 & I & B \\
0 & I & 0 & 0 \\
0 & 0 & I & 0 \\
0 & I & 0 & I
\end{array}\right)=\left(\begin{array}{cccc}
0 & I & 0 & 0 \\
I & 0 & 0 & 0 \\
0 & 0 & 0 & -I \\
0 & 0 & -I & -B
\end{array}\right),
$$

o que mostra que $\mathbb{M}(U, b) \perp \mathbb{M}(U,-b) \simeq \mathbb{H}(U) \perp \mathbb{M}(U, b)$, como queríamos. 


\subsection{Cancelamento de Witt}

Nesta seção apresentaremos um importante resultado sobre espaços quadráticos, o Teorema do Cancelamento de Witt, o qual será usado no capítulo final de nosso trabalho. Para tanto, apresentaremos algumas isometrias de certos tipos de espaços quadráticos.

Seja $(M, q)$ um espaço quadrático sobre o anel $A$. Para quaisquer $x, y \in M$, tais que $q(x)=0$ e $b_{q}(x, y)=0$, isto é, $y \in(A x)^{\perp}$, indiquemos por $E(x, y)$ a aplicação $A$-linear $E(x, y): M \rightarrow M$, dada por:

$$
E(x, y)(z)=z+b_{q}(z, x) y-b_{q}(z, y) x-q(y) b_{q}(z, x) x
$$

para todo $z \in M$.

Se $M$ tem uma decomposição $M \simeq A u \oplus A v \perp M_{0}$, onde $A u \oplus A v$ é um plano hiperbólico e $M_{0} \neq\{0\}$, então para cada $\lambda \in A^{\star}$, indiquemos por $\phi(\lambda)$ a aplicação $A$-linear $\phi(\lambda): M \rightarrow M$, dada por:

$$
\phi(\lambda)(u)=\lambda u, \quad \phi(\lambda)(v)=\lambda^{-1} v \quad \text { e } \quad \phi(\lambda)(x)=x
$$

para todo $x \in M_{0}$.

Com simples cálculos mostra-se que $q(E(x, y)(z))=q(z)$, para todo $z \in M$. Se $y^{\prime} \in(A x)^{\perp}$ então

$$
E(x, y) \circ E\left(x, y^{\prime}\right)=E\left(x, y+y^{\prime}\right)
$$

Em particular, $E(x, y) \circ E(x,-y)=i d_{M}$, ou seja $E(x, y)$ é uma isometria de $(M, q)$ chamada de Transveç̧ão de Siegel. É fácil ver que as aplicações $\phi(\lambda)$, com $\lambda \in A^{\star}$, são também isometrias de $(M, q)$.

Os próximos resultados serão usados na demonstração do Teorema do Cancelamento de Witt para espaços quadráticos.

Lema 2.32 Sejam $A$ um anel semilocal e $(M, q) \in Q u a d(A)$ com uma decomposição $M \simeq$ $A u \oplus A v \perp M_{0}$, onde $A u \oplus A v$ é um plano hiperbólico e $M_{0} \neq\{0\}$. Seja $x=\alpha u+\beta v+z$; $\alpha, \beta \in A$ e $z \in M_{0}$, um vetor primitivo de $M$. Assumamos uma das seguintes condições:

(i) $|A / \mathrm{m}| \geq 3$, para todo $\mathrm{m} \in \operatorname{Spm}(A)$;

(ii) $x$ é isotrópico.

Então existe $y \in M_{0}$ tal que $E(u, y)(x)=\alpha^{\prime} u+\beta v+z^{\prime}$, com $\alpha^{\prime} \in A^{\star}$ e $z^{\prime} \in M_{0}$. 
Dem.: Desde que $E(u, y)(x)=\left(\alpha-b_{q}(y, z)-q(y) \beta\right) u+\beta v+(z+\beta y)$, basta mostrarmos que existe $y \in M_{0}$ tal que $\left(\alpha-b_{q}(y, z)-q(y) \beta\right) \in A^{\star}$. Isto significa que

$$
\left(\alpha-b_{q}(y, z)-q(y) \beta\right) \notin \mathfrak{m}
$$

para todo $\mathrm{m} \in \operatorname{Spm}(A)$.

Fixando um $\mathrm{m} \in \operatorname{Spm}(A)$, denotamos as classes residuais de $\alpha, \beta, \ldots, y, z, \ldots, \mathrm{em} A / \mathrm{m}$ e em $M_{0}(\mathfrak{m})$ por $\bar{\alpha}, \bar{\beta}, \ldots, \bar{y}, \bar{z}, \ldots$, respectivamente. Mostremos que existe $\bar{y} \in M_{0}(\mathfrak{m})$ tal que $\bar{\alpha}-b_{q}(\bar{y}, \bar{z})-q(\bar{y}) \bar{\beta} \neq \overline{0}$ em $A / \mathrm{m}$. Se $\bar{\alpha} \neq \overline{0}$ em $A / \mathrm{m}$, tomamos $\bar{y}=\overline{0}$. Se $\bar{\alpha}=\overline{0}$, queremos encontrar $\bar{y} \in M_{0}(\mathrm{~m})$ tal que $b_{q}(\bar{y}, \bar{\beta}) \neq \overline{0}$ em $A / \mathrm{m}$.

Assumindo a condição (ii), isto é, $x$ isotrópico, temos que $\bar{x}$ é isotrópico em $M(\mathrm{~m})$. Logo $q(\bar{x})=q(\bar{\beta} \bar{v}+\bar{z})=q(\bar{z})=\overline{0}$. Se $\bar{z}=\overline{0}$, então $\bar{\beta} \neq \overline{0}$ pois $x$ é primitivo. Neste caso, é suficiente tomarmos $\bar{y} \in M_{0}(\mathrm{~m})$ com $q(\bar{y}) \neq \overline{0}$, que existe pela não singularidade de $M_{0}(\mathrm{~m})$. Se $\bar{z} \neq \overline{0}$, então $\bar{z}$ é um vetor isotrópico de $M_{0}(\mathfrak{m})$. Neste caso, existe $\bar{y} \in M_{0}(\mathfrak{m})$ tal que $q(\bar{y})=\overline{0}$ e $b_{q}(\bar{y}, \bar{z})=\overline{1}$, pois $M_{0}(\mathrm{~m})$ é isotrópico. Assim mostramos que com a condição (ii), existe $\bar{y} \in M_{0}(\mathrm{~m})$ tal que $\bar{\alpha}-b_{q}(\bar{y}, \bar{z})-q(\bar{y}) \bar{\beta} \neq \overline{0} \mathrm{em} A / \mathrm{m}$.

Assumindo a condição $(i)$, consideremos $\left\{x_{1}, \ldots, x_{n}\right\}$ uma base de $\left(M_{0}, q\right)$. Então $\left\{\overline{x_{1}}, \ldots, \overline{x_{n}}\right\}$ é uma base de $M_{0}(\mathfrak{m})$ e, neste caso, queremos encontrar $\bar{y}=\sum_{i=1}^{n} \overline{\lambda_{i}} \overline{x_{i}}$ em $M_{0}(\mathrm{~m})$, ou seja, encontrar $\overline{\lambda_{1}}, \ldots, \overline{\lambda_{n}} \in A / \mathrm{m}$ tais que o polinômio

$$
f\left(\overline{\lambda_{1}}, \ldots, \overline{\lambda_{n}}\right)=\sum_{i=1}^{n} b_{q}\left(\overline{x_{i}}, \bar{z}\right) \overline{\lambda_{i}}+\bar{\beta} \sum_{i, j=1, i \neq j}^{n} b_{q}\left(\overline{x_{i}}, \overline{x_{j}}\right) \overline{\lambda_{i} \lambda_{j}}+\bar{\beta} \sum_{i=1}^{n} q\left(\overline{x_{i}}\right)\left(\overline{\lambda_{i}}\right)^{2} \neq \overline{0} \in A / \mathrm{m} .
$$

Como $x$ é primitivo e $\left\{x_{1}, \ldots, x_{n}\right\}$ é uma base de $\left(M_{0}, q\right)$, temos que $f$ é um polinômio não nulo em $n$ variáveis, com coeficientes em $A / \mathrm{m}$ e grau $\leq 2$. Como $|A / \mathrm{m}| \geq 3$, temos que $f$ representa um elemento não nulo em $A / \mathrm{m}$. Assim, assumindo a condição (i), mostramos a existência de $\bar{y} \in M_{0}(\mathrm{~m})$ tal que $b_{q}(\bar{y}, \bar{z})-q(\bar{y} \bar{\beta}) \neq \overline{0} \mathrm{em} A / \mathrm{m}$.

Portanto, para cada $m \in S p m(A)$, construímos um elemento $\overline{y_{m}} \in M_{0}(\mathfrak{m})$ com $\bar{\alpha}-b_{q}\left(\check{y}_{\mathfrak{m}}, \bar{z}\right)-q\left(\overline{y_{m}}\right) \bar{\beta} \neq \overline{0}$ em $A / \mathrm{m}$. Desde que $A$ é um anel semilocal, usando o Teorema Chinês dos Restos, podemos encontrar $y \in M_{0}$, com $y \equiv y_{\mathfrak{m}} \bmod \mathfrak{m}$, para cada $\mathrm{m} \in \operatorname{Spm}(A)$. De (1.3) temos que este elemento $y$ satisfaz o requerido.

Sejam $(M, q) \in \mathcal{Q u a d}(A)$ e $\left(E,\left.q\right|_{E}\right)$ um subespaço de $(M, q)$. Um monomorfismo de $A$-módulos $\varphi: E \rightarrow M$, tal que $q(\varphi(x))=q(x)$ para todo $x \in E$ e $\varphi(E)$ é um subespaço de $M$, também é dito ser uma isometria. 
Lema 2.33 Sejam $A$ um ancl semilocal, $(M, q) \in Q$ uad $(A)$ com uma decomposição como em (2.32) e $\mathbb{H} \subseteq M$ um plano hiperbólico. Então existe uma isometria $\varphi: M \rightarrow M$ que leva $(A u \oplus A v)$ em $\mathbf{H}$.

Dem.: Seja $\{x, y\}$ uma base para $\mathbb{H}, \operatorname{com} q(x)=q(y)=0$ e $b_{q}(x, y)=1$. O elemento $x$ é da forma $\alpha u+\beta v+z$, com $\alpha, \beta \in A$ e $z \in M_{0}$. Usando o fato que $x$ é primitivo e isotrópico, segue de (2.32) que podemos supor $\alpha \in A^{\star}$.

Observe que $E\left(v, \alpha^{-1} z\right)(u)=u+\alpha^{-1} z-\alpha^{-2} q(z) v$. Como $0=q(x)=\alpha \beta+q(z)$ e $\alpha \in A^{\star}$, temos que $\beta=-\alpha^{-1} q(z)$ e, conseqüentemente

$$
E\left(v, \alpha^{-1} z\right)(u)=u+\alpha^{-1} z+\alpha^{-1} \beta v=\alpha^{-1}(\alpha u+\beta v+z)=\alpha^{-1} x
$$

Tomando a isometria $\psi=E\left(v, \alpha^{-1} z\right) \circ \phi(\alpha)$, temos $\psi(u)=x$, ou seja, $\psi$ leva $(A u \oplus$ $A v)$ em $A x \oplus A \psi(v)$ que é um plano hiperbólico de $M$. Considerando $w=\psi(v)$ e a decomposição $M \simeq(A x \oplus A w) \perp M_{1}$, temos que o elemento $y \in M$ pode ser escrito na forma $y=\lambda x+\gamma y+z^{\prime}, \operatorname{com} \lambda, \gamma \in A$ e $z^{\prime} \in M_{1}$. Desde que $1=b_{q}(x, y)=b_{q}(\psi(x), \psi(y))=b_{q}(x, w)$ e $y$ é isotrópico, obtemos $\gamma=1$ e $\lambda=-q\left(z^{\prime}\right)$. Então $E\left(x, z^{\prime}\right)(w)=-q\left(z^{\prime}\right) x+w+z^{\prime}=y$ e $E\left(x, z^{\prime}\right)(x)=x$. Logo $\varphi=E\left(x, z^{\prime}\right) \circ \psi$ satisfaz o requerido.

Agora o Teorema do Cancelamento de Witt para espaços quadráticos segue como conseqüência do teorema abaixo.

Teorema 2.34 Sejam $A$ um anel semilocal, $(M, q) \in \operatorname{Quad}(A)$ e $\left(E,\left.q\right|_{E}\right)$ um subespaço não singular de $(M, q)$. Cada isometria $\varphi: E \rightarrow M$ pode ser estendida a uma isometria $\phi: M \rightarrow M$.

Dem.: Por (2.16) temos que $M=E \perp E^{\perp}$. Consideremos o espaço $M \perp-E \simeq$ $E \perp-E \perp E^{\perp} \simeq \mathbb{H}[E] \perp E^{\perp}$. A isometria $\varphi: E \rightarrow M$ induz uma isometria $\varphi^{\prime}=\varphi \perp i d_{-E}: \mathbb{H}[E] \rightarrow M \perp-E$ e, cada extensão $\tilde{\varphi}^{\prime}$ de $\varphi^{\prime}$ à $M \perp-E$ nos dá uma extensão $\operatorname{de} \varphi$ à $M$. De fato, basta observarmos que $(-E)^{\perp} \cong M$ em $M \perp-E$ e $\left.\widetilde{\varphi}^{\prime}\right|_{-E}=\left.\varphi^{\prime}\right|_{-E}=i d_{-E}$ donde segue que $\widetilde{\varphi}^{\prime}$ é uma isometria de $(-E)^{\perp}$ nele mesmo, ou seja, $\left.\widetilde{\varphi}^{\prime}\right|_{M}$ é uma extensão de $\varphi$ à $M$. Assim, podemos supor que $E$ é hiperbólico, isto é, que $E=\mathbb{H}_{1} \perp \ldots \perp \mathbb{H}_{n}$, onde $\mathbb{H}_{i}=A u_{i} \oplus A v_{i}$ são planos hiperbólicos.

Agora usaremos indução sobre $n$ para obtermos o resultado. Se $n=1,(2.33)$ garante a existência da extensão de $\varphi$. Suponhamos $n>1$ e consideremos a restrição de $\varphi$, 
$\varphi_{1}: \mathbb{H}_{1} \perp \ldots \perp \mathbb{H}_{n-1} \rightarrow M$. Por hipótese de indução, existe uma isometria $\sigma: M \rightarrow M$ que estende $\varphi_{1}$. Então $\sigma^{-1} \circ \varphi: E \rightarrow M$ é uma isometria que é a identidade em $\mathbb{H}_{1} \perp \ldots \perp H_{n-1}$. Logo a restrição de $\sigma^{-1} \circ \varphi$ à $\mathbb{H}_{n}$ é uma isometria $\sigma^{-1} \circ \varphi: \mathbb{H}_{n} \rightarrow\left(\mathbb{H}_{1} \perp \ldots \perp \mathbb{H}_{n-1}\right)^{\perp}$. Como $\mathbb{H}_{n} \subseteq\left(\mathbb{H}_{1} \perp \ldots \perp \mathbb{H}_{n-1}\right)^{\perp}$, segue do observado acima que $\sigma^{-1} \circ \varphi$ se estende a uma isometria $\tau$ de $\left(\mathbb{H}_{1} \perp \ldots \perp \mathbb{H}_{n-1}\right)^{\perp}$, logo $\psi=i d_{\mathbf{H}_{1} \perp \ldots \perp H_{n-1}} \perp \tau: M \rightarrow M$ é uma extensão de $\tau$ a $M$ tal que $\left.\psi\right|_{\mathbf{H}_{1} \perp \ldots \perp \mathbf{H}_{n-1}}=i d_{\mathbf{H}_{1} \perp \ldots \perp \mathbf{H}_{n-1}}$. Agora é imediato ver que $\phi=\sigma \circ \psi: M \rightarrow M$ é uma extensão de $\varphi$, como queríamos.

Corolário 2.35 (Teorema do Cancelamento de Witt) $\operatorname{Sejam}\left(M_{i}, q_{i}\right), i=1,2,3$, espaços quadráticos sobre um anel semilocal $A$ tais que $M_{1} \perp M_{2} \simeq M_{1} \perp M_{3}$. Então $M_{2} \simeq M_{3}$.

Dem.: Sejam $\sigma: M_{1} \perp M_{2} \rightarrow M_{1} \perp M_{3}$ uma isometria e $\iota: M_{1} \rightarrow M_{1} \perp M_{2}$ a inclusão. De (2.34) segue que $\sigma \circ \iota: M_{1} \rightarrow M_{1} \perp M_{3}$ admite uma extensão à uma isometria $\psi: M_{1} \perp M_{3} \rightarrow M_{1} \perp M_{3}$. Assim a isometria

$$
\varphi=\sigma^{-1} \circ \psi: M_{1} \perp M_{3} \rightarrow M_{1} \perp M_{2}
$$

satisfaz $\left.\varphi\right|_{M_{1}}=i d_{M_{1}}$, o que implica em $\left.\varphi\right|_{M_{3}}$ ser uma isometria de $M_{3}$ em $M_{2}$, isto é, $M_{2} \simeq M_{3}$, como queríamos.

Observação 2.36 O Teorema do Cancelamento de Witt não vale em geral para espaços bilineares sobre um anel semilocal $A$. Por exemplo se $A$ é um corpo de característica 2, temos $\langle 1,1,1,1\rangle \simeq\langle 1,1\rangle \perp\left(\begin{array}{ll}0 & 1 \\ 1 & 0\end{array}\right)$ e $\langle 1,1\rangle$ não é isométrico a $\left(\begin{array}{ll}0 & 1 \\ 1 & 0\end{array}\right)$. Mas, se $2 \in A^{\star}$ segue da observação (2.3) que o Teorema do Cancelamento de Witt vale em $\operatorname{Bil}(A)$. 


\section{Capítulo 3}

\section{O Anel de Witt}

Neste capítulo apresentaremos um estudo da estrutura dos anéis de Witt dos espaços bilineares, $\mathcal{W}(A)$, e dos espaços quadráticos, $\mathcal{W}_{Q}(A)$, sobre um anel semilocal $A$. Mais especificamente, daremos uma descrição dos ideais primos e dos geradores de $\mathcal{W}(A)$, faremos um estudo dos elementos nilpotentes e dos elementos de torção de $\mathcal{W}(A)$ e daremos alguns resultados sobre $\mathcal{W}_{Q}(A)$ necessários no próximo capítulo. Em todo capítulo, salvo menção contrária, $A$ denotará sempre um anel semilocal.

\subsection{Os Anéis $\mathcal{W}(A)$ e $\mathcal{W}_{Q}(A)$}

Às categorias $\operatorname{Bil}(A)$ e $\operatorname{Quad}(A)$ associamos seus correspondentes anéis de Grothendieck, $K_{0}(\mathcal{B} i l(A))$ e $K_{0}(\operatorname{Quad}(A))$ os quais são chamados, respectivamente, anel de Witt-Grothendieck dos espaços bilineares sobre $A$ e anel de Witt-Grothendieck dos espaços quadráticos sobre $A$. Tais anéis são denotados respectivamente por, $\widehat{\mathcal{W}}(A)$ e $\widehat{\mathcal{W}_{Q}}(A)$.

$\mathrm{Se}[b]$ denota a classe de isometrias do espaço bilinear $b$, então os elementos de $\widehat{\mathcal{W}}(A)$ são as diferenças formais $\left[b_{1}\right]-\left[b_{2}\right]$, de classes $\left[b_{1}\right]$ e $\left[b_{2}\right]$, onde por definição $\left[b_{1}\right]-\left[b_{2}\right]=\left[b_{1}^{\prime}\right]-\left[b_{2}^{\prime}\right]$ se, e somente se existe $b \in \operatorname{Bil}(A)$ tal que $b_{1} \perp b_{2}^{\prime} \perp b \simeq b_{2} \perp b_{1}^{\prime} \perp b$. Respectivamente, os elementos de $\widehat{\mathcal{W}_{Q}}(A)$ são diferenças formais $\left[q_{1}\right]-\left[q_{2}\right]$ de classes de isometrias de espaços quadráticos. Observemos que no caso quadrático, vale o Teorema do Cancelamento de Witt, confira (2.35), e conseqüentemente, $\left[q_{1}\right]-\left[q_{2}\right]=\left[q_{1}^{\prime}\right]-\left[q_{2}^{\prime}\right]$ se , e somente se $q_{1} \perp q_{2}^{\prime} \simeq q_{1}^{\prime} \perp q_{2}$.

Considere os seguintes conjuntos

$$
\begin{aligned}
& \widehat{\mathbb{M}}(A)=\left\{[b]-\left[b^{\prime}\right] \in \widehat{\mathcal{W}}(A) ; b \text { e } b^{\prime} \quad \text { são metábolicos }\right\}, \\
& \widehat{\mathbb{H}}[A]=\left\{[q]-\left[q^{\prime}\right] \in \widehat{\mathcal{W}}_{Q}(A) ; q \text { e } q^{\prime} \quad \text { são hiperbólicos }\right\} .
\end{aligned}
$$


De (2.30), $(i i i)$, decorre que $\widehat{\mathbb{M}}(A)$ e $\widehat{H}[A]$ são ideais de $\widehat{\mathcal{W}}(A)$ e $\widehat{\mathcal{W}}_{Q}(A)$, respectivamente. Assim definimos, $\mathcal{W}(A)=\frac{\widehat{W}(A)}{\widehat{\mathbb{M}}(A)}$, o anel de Witt dos espaços bilineares sobre $A \mathrm{e}$ $W_{Q}(A)=\frac{\widehat{\mathcal{W}}_{q}(A)}{\widehat{\mathbb{H}}[A]}$, o anel de Witt dos espaços quadráticos sobre $A$, onde as operações de $\mathcal{W}(A)$ são induzidas por $\perp, \oplus, \mathrm{e} \perp, \odot$ induzem as operaçoes de $\mathcal{W}_{Q}(A)$.

Com a mesma notação de $\widehat{\mathcal{W}}(A)$, seja $\left[b_{1}\right]-\left[b_{2}\right]$ um elemento genérico de $\mathcal{W}(A)$. Então

$$
\left[b_{1}\right]-\left[b_{2}\right]=\left[b_{1}\right]-\left[b_{2}\right]+\left[\left(-b_{2}\right)\right]-\left[\left(-b_{2}\right)\right]=\left[b_{1} \perp\left(-b_{2}\right)\right]-\left[b_{2} \perp\left(-b_{2}\right)\right] .
$$

De (2.30), (ii), temos que $b_{2} \perp\left(-b_{2}\right)$ é metabólico; logo $\left[b_{2} \perp\left(-b_{2}\right)\right]=[0]$ em $\mathcal{W}(A)$. Assim, $\left[b_{1}\right]-\left[b_{2}\right]=\left[b_{1} \perp\left(-b_{2}\right)\right]$, ou seja, todo elemento de $\mathcal{W}(A)$ é da forma $[b]$ com $b \in \operatorname{Bil}(A)$. De maneira análoga, vemos que os elementos de $\mathcal{W}_{Q}(A)$ são da forma $[q] \operatorname{com} q \in Q$ uad $(A)$.

Da definição de $\mathcal{W}(A)$ segue que $\left[b_{1}\right]=\left[b_{2}\right]$ se, e somente se existem $U, V$ módulos bilineares tais que $b_{1} \perp \mathbb{M}(U) \simeq b_{2} \perp \mathbb{M}(V)$.

$O$ anel $\mathcal{W}(A)$ possui um elemento identidade, a saber $1_{\mathcal{W}(A)}=[\langle 1\rangle]$. Enquanto que, se $2 \notin A^{\star}$, o anel $\mathcal{W}_{Q}(A)$ não possui elemento identidade. Se $2 \in A^{\star}$, com a observação (2.3), podemos identificar $\mathcal{W}(A)$ e $\mathcal{W}_{Q}(A)$. Em geral, usando $(\mathbf{2 . 3 0}),(i i i), \mathcal{W}_{Q}(A)$ é visto como uma $\mathcal{W}(A)$-álgebra. Quando não houver perigo de confusão denotaremos simplesmente por $b$ o elemento $[b]$ de $\mathcal{W}(A)$, dentro do contexto se tornará claro quando considerarmos $b$ como um elemento de $\mathcal{W}(A)$ ou como um elemento de $\operatorname{Bil}(A)$. Respectivamente $q$ denotará o elemento $[q]$ de $\mathcal{W}_{Q}(A)$.

Decorre de (2.31) que, para todo módulo bilinear $(U, b), \mathbb{M}(U)=\mathbb{H}(U)$ em $\widehat{\mathcal{W}}(A)$. Desta forma se considerarmos o subconjunto de $\widehat{\mathcal{W}}(A)$

$$
\widehat{\mathbb{H}}(A)=\{[\mathbb{H}(U)]-[\mathbb{H}(V)] ; U, V \text { são módulos bilineares }\} \text {, }
$$

temos que $\widehat{\mathrm{H}}(A)=\widehat{\mathbb{M}}(A)$ em $\widehat{\mathcal{W}}(A)$. Agora se $\operatorname{dim}(U)=m$ e $\operatorname{dim}(V)=n$ temos que $\mathrm{H}(U) \simeq m \mathbb{H}$ e $\mathbf{H}(V) \simeq n H$, ou seja em $\widehat{W}(A)$ temos

$$
[\mathbb{H}(U)]-[\mathbb{H}(V)]=[m \mathbb{H}]-[n \mathbb{H}]=[(m-n) \mathbb{H}],
$$

com $m-n \in \mathbb{Z}$. Portanto, podemos identificar $\widehat{\mathbb{H}}(A)$ com $\mathbb{Z} H=\{n \mathbb{H} ; n \in \mathbb{Z}\}$ e escrever $\mathcal{W}(A)=\frac{\widehat{W}(A)}{\mathbb{Z} \mathbb{H}}$.

Proposição 3.1 Dois espaços bilineares são iguais em $\widehat{\mathcal{W}}(A)$ se, e somente se são iguais em $\mathcal{W}(A)$ e tem a mesma dimensão. 
Dem.: Se $\left[b_{1}\right]=\left[b_{2}\right]$ em $\widehat{W}(A)$, então existe $(M, b) \in \operatorname{Bil}(A)$ tal que $b_{1} \perp b \simeq b_{2} \perp b$ e, conseqüentemente, $\operatorname{dim}\left(b_{1}\right)=\operatorname{dim}\left(b_{2}\right)$. Além disso temos $b_{1} \perp b \perp(-b) \simeq b_{2} \perp b \perp(-b)$ o que implica, de (2.30),(ii), que $b_{1} \perp \mathbb{M}(V) \simeq b_{2} \perp \mathbb{M}(V)$, ou seja, $\left[b_{1}\right]=\left[b_{2}\right]$ em $\mathcal{W}(A)$. Reciprocamente, sejam $b_{1}, b_{2} \in \mathcal{B} i l(A)$ espaços de mesma dimensão tais que $\left[b_{1}\right]=\left[b_{2}\right]$ em $\mathcal{W}(A)$. Então existem $m, n \in \mathbb{Z}$ tais que $b_{1} \perp m \mathbb{H} \simeq b_{2} \perp n \mathbb{H}$. Como $\operatorname{dim}\left(b_{1}\right)=\operatorname{dim}\left(b_{2}\right)$, igualando as dimensões temos que $m=n$. Portanto, $\left[b_{1}\right]=\left[b_{2}\right]$ em $\widehat{\mathcal{W}}(A)$.

\subsection{Geradores de $\mathcal{W}(A)$}

Sejam $G=A^{\star} /\left(A^{\star}\right)^{2}$ o grupo das classes quadradas de $A$ e $f: G \rightarrow \mathcal{W}(A)$ a aplicação que leva cada classe $(\alpha) \in G$ no elemento $[\langle\alpha\rangle] \in \mathcal{W}(A)$. Escrevemos $f(\alpha)$ para indicar a imagem de $(\alpha)$ pela aplicação $f$.

Como um primeiro resultado sobre a geração do anel de Witt temos

Teorema $3.2 O$ anel $\mathcal{W}(A)$ é aditivamente gerado por $f(G)$, a imagem de $f$.

Dem.: Seja $b \in \operatorname{Bil}(A)$. O espaço $b \perp\langle 1\rangle$ é próprio e, de (2.20), $b \perp\langle 1\rangle$ admite uma base ortogonal, isto é, existem $\beta_{1}, \ldots, \beta_{n} \in A^{\star}$ tais que:

$$
b \perp\langle 1\rangle \simeq\left\langle\beta_{1}\right\rangle \perp \ldots \perp\left\langle\beta_{n}\right\rangle
$$

Desde que $\langle 1,-1\rangle$ é metabólico, em $\mathcal{W}(A)$ temos:

$$
b=b \perp\langle 1,-1\rangle=\left\langle\beta_{1}\right\rangle \perp \ldots \perp\left\langle\beta_{n}\right\rangle \perp\langle-1\rangle=f\left(\beta_{1}\right) \perp \ldots \perp f\left(\beta_{n}\right) \perp f(-1) .
$$

Assim, todo elemento de $\mathcal{W}(A)$ se escreve como uma soma finita de elementos de $f(G)$.

Observação 3.30 resultado acima mostra, em particular, que todo elemento de $\mathcal{W}(A)$ pode ser representado pela classe de um espaço bilinear próprio sobre $A$, independentemente de 2 ser ou não invertível no anel $A$.

Seja $\mathbb{Z}[G]$ o anel de grupos de $G$. Também do teorema anterior, podemos afirmar que existe um homomorfismo de anéis sobrejetor $\varphi: \mathbb{Z}[G] \rightarrow \mathcal{W}(A)$, que é a extensão por linearidade de $f$. $O$ próximo resultado caracteriza o núcleo $\mathcal{K}$ deste homomorfismo. 
Proposição 3.4 $O$ ideal $\mathcal{K}$ de $\mathbb{Z}[G]$ é aditivamente gerado por $(1)+(-1)$ e por todos os elementos da forma $\sum_{i=1}^{4}\left(\alpha_{i}\right)-\sum_{i=1}^{4}\left(\beta_{i}\right) \in \mathbb{Z}[G]$, tais que

$$
\left\langle\alpha_{1}, \alpha_{2}, \alpha_{3}, \alpha_{4}\right\rangle \simeq\left\langle\beta_{1}, \beta_{2}, \beta_{3}, \beta_{4}\right\rangle \text {. }
$$

Dem.: Claramente os elementos deste tipo estão em $\mathcal{K}$. Reciprocamente, seja $z=\sum_{i=1}^{r}\left(\alpha_{i}\right)-\sum_{i=1}^{s}\left(\beta_{i}\right)$ um elemento de $\mathcal{K}$. Trocando $z$ por $-z$ se necessário, podemos assumir que $r \geq s$. Desde que $\varphi(z)=0$, temos que $\left\langle\alpha_{1}, \ldots, \alpha_{r}\right\rangle=\left\langle\beta_{1}, \ldots, \beta_{s}\right\rangle$ em $\mathcal{W}(A)$, ou seja, existem $U_{1}, U_{2} \in \operatorname{Bil}(A)$, tais que

$$
\left\langle\alpha_{1}, \ldots, \alpha_{r}\right\rangle \perp \mathbb{M}\left(U_{1}\right) \simeq\left\langle\beta_{1}, \ldots, \beta_{s}\right\rangle \perp \mathbb{M}\left(U_{2}\right) .
$$

Como, $\operatorname{dim}\left(\mathbb{M}\left(U_{1}\right)\right)$ e $\operatorname{dim}\left(\mathbb{M}\left(U_{2}\right)\right)$ são números pares, temos que $r-s$ é um número par, digamos $2 t, \operatorname{com} t \geq 0$.

Sejam $b_{1}=\left\langle\alpha_{1}, \ldots, \alpha_{r}\right\rangle$ e $b_{2}=\left\langle\beta_{1}, \ldots, \beta_{s}\right\rangle \perp t\langle 1,-1\rangle$. Desde que $b_{1}=b_{2}$ em $\mathcal{W}(A)$ e $\operatorname{dim}\left(b_{1}\right)=\operatorname{dim}\left(b_{2}\right)$, temos por (3.1) que eles representam o mesmo elemento em $\widehat{\mathcal{W}}(A)$. Assim, existe $b_{3} \in \mathcal{B} i l(A)$ tal que

$$
\left\langle\alpha_{1}, \ldots, \alpha_{r}\right\rangle \perp b_{3} \simeq\left\langle\beta_{1}, \ldots, \beta_{s}\right\rangle \perp t\langle 1,-1\rangle \perp b_{3} .
$$

Somando $\langle 1\rangle$ em ambos os lados, se necessário, podemos assumir que $b_{3}$ é próprio, ou seja $b_{3} \simeq\left\langle\alpha_{r+1}, \ldots, \alpha_{n}\right\rangle$. Tomando $\beta_{i}= \pm 1$ para $s<i \leq r$ e $\beta_{i}=\alpha_{i}$ para $r<i \leq$ $n$, obtemos $\left\langle\alpha_{1}, \ldots, \alpha_{n}\right\rangle \simeq\left\langle\beta_{1}, \ldots, \beta_{n}\right\rangle$ e $z=t((1)+(-1))+\sum_{i=1}^{n}\left(\alpha_{i}\right)-\sum_{i=1}^{n}\left(\beta_{i}\right)$. Seja $z^{\prime}=\sum_{i=1}^{n}\left(\alpha_{i}\right)-\sum_{i=1}^{n}\left(\beta_{i}\right)$, com $\left\langle\alpha_{1}, \ldots, \alpha_{n}\right\rangle \simeq\left\langle\beta_{1}, \ldots, \beta_{n}\right\rangle$. Então existe um espaço bilinear próprio $(M, b)$ sobre $A$, com bases ortogonais $\mathcal{B}=\left\{x_{1}, \ldots, x_{n}\right\}$ e $\mathcal{B}^{\prime}=\left\{y_{1}, \ldots, y_{n}\right\}$ tais que $b\left(x_{i}, x_{i}\right)=\alpha_{i}$ e $b\left(y_{i}, y_{i}\right)=\beta_{i}$, para todo $i=1, \ldots, n$. Do teorema $(2.25)$ temos que $\mathcal{B}$ está 4-relacionada com $\mathcal{B}^{\prime}$. Seja $\mathcal{B}=\mathcal{B}_{1}, \mathcal{B}_{2}, \ldots, \mathcal{B}_{k}=\mathcal{B}^{\prime}$ uma seqüência de bases ortogonais de $(M, q)$ efetivando a 4-relação. Escrevendo $\mathcal{B}_{i}=\left\{w_{i_{1}}, \ldots, w_{i_{n}}\right\}$ e $\lambda_{i j}=b\left(w_{i_{j}}, w_{i_{j}}\right) \in A^{\star} \mathrm{e}$, denotando o elemento $\sum_{j=1}^{n}\left(\lambda_{i j}\right) \in \mathbb{Z}[G]$ por $\left(B_{i}\right)$, temos

$$
z^{\prime}=\left(\left(B_{1}\right)-\left(B_{2}\right)\right)+\left(\left(B_{2}\right)-\left(B_{3}\right)\right)+\ldots+\left(\left(B_{k-1}\right)-\left(B_{k}\right)\right),
$$

que é da forma requerida. 
Teorema 3.5 $O$ anel $\mathcal{W}(A)$ é aditivamente gerado por $\left\{\langle\alpha\rangle ; \alpha \in A^{\star}\right\}$ com as seguintes relações:

(i) $\left\langle\alpha \beta^{2}\right\rangle=\langle\alpha\rangle$ para todo $\beta \in A^{\star}$;

(ii) $\left\langle\alpha_{1}\right\rangle+\cdots+\left\langle\alpha_{n}\right\rangle=\left\langle\beta_{1}\right\rangle+\cdots+\left\langle\beta_{n}\right\rangle \Leftrightarrow\left\langle\alpha, \ldots, \alpha_{n}\right\rangle \simeq\left\langle\beta_{1}, \ldots, \beta_{n}\right\rangle$;

(iii) $\langle\alpha\rangle+\langle-\alpha\rangle=0$;

(iv) $\langle\alpha\rangle+\langle\beta\rangle=\langle\alpha+\beta\rangle+\langle\alpha \beta(\alpha+\beta)\rangle$, se $\alpha+\beta \in A^{\star}$;

(v) $\langle\alpha\rangle\langle\beta\rangle=\langle\alpha \beta\rangle$.

Dem.: Para mostrarmos que (i), (ii), (iii) e $(v)$ valem para $\mathcal{W}(A)$, basta observarmos que $\mathcal{W}(A) \cong \frac{\mathbb{Z}[G]}{\mathcal{K}}$ e usarmos o teorema anterior.

Mostremos então o item (iv). Consideremos o espaço bilinear $(M, b)=\langle\alpha, \beta\rangle$ com uma base $\{x, y\}$ tal que, $b(x, y)=0, b(x, x)=\alpha$ e $b(y, y)=\beta$. Então, $b(x+y, x+y)=$ $\alpha+\beta \in A^{\star}$. Como $\alpha+\beta \in A^{\star}$ temos que $A(x+y)$ é um subespaço não singular de $M \mathrm{e}$ de (2.16), $M=A(x+y) \perp(A(x+y))^{\perp}$. Como $(A(x+y))^{\perp}$ é um subespaço não singular de $(M, b)$ unidimensional, existe $z \in M \operatorname{com} b(z, z)=\gamma \in A^{*}$ e $b(x+y, z)=0$, isto é, $(A(x+y))^{\perp}=A z$. Assim,

$$
\langle\alpha\rangle \perp\langle\beta\rangle \simeq\langle\alpha+\beta\rangle \perp\langle\gamma\rangle \text {. }
$$

Comparando os determinantes, temos $\alpha \beta \equiv(\alpha+\beta) \gamma \bmod \left(A^{\star}\right)^{2}$. Isto implica que $\alpha \beta(\alpha+\beta)^{-1} \equiv \gamma \bmod \left(A^{\star}\right)^{2}$, ou seja, $\gamma \equiv \alpha \beta(\alpha+\beta) \bmod \left(A^{\star}\right)^{2}$. Logo, de $(i)$, $\langle\gamma\rangle=\langle\alpha \beta(\alpha+\beta)\rangle$. Conseqüentemente,

$$
\langle\alpha\rangle \perp\langle\beta\rangle \simeq\langle\alpha+\beta\rangle \perp\langle\alpha \beta(\alpha+\beta)\rangle .
$$

Portanto, $\langle\alpha\rangle+\langle\beta\rangle=\langle\alpha+\beta\rangle+\langle\alpha \beta(\alpha+\beta)\rangle$, em $\mathcal{W}(A)$, por (ii).

\subsection{Os ideais primos de $\mathcal{W}(A)$}

Nesta seção caracterizaremos os ideais primos de $\mathcal{W}(A)$ usando o isomorfismo de anéis $\mathcal{W}(A) \cong \frac{\mathbb{Z}[G]}{\mathcal{K}}$, onde $G=A^{\star} /\left(A^{\star}\right)^{2}$ e $\mathcal{K}$ é bem determinado em (3.4), ou seja, usaremos 
o fato que os ideais primos de $\mathcal{W}(A)$ estão em correspondência biunívoca com os ideais primos de $\mathbb{Z}[G]$ que contém $\mathcal{K}$.

Para tal fim começaremos determinando todos os ideais primos de $\mathbb{Z}[G]$ e, a seguir aqueles que contém $\mathcal{K}$.

Lema 3.6 Para cada ideal primo p de $\mathbb{Z}[G]$, temos:

(i) Se $\mathbb{Z} \cap \mathfrak{p}=\{0\}$, então existe um único homomorfismo de anéis $\phi$ de $\mathbb{Z}[G]$ em $\mathbb{Z}$ com núcleo $\mathfrak{p}$;

(ii) Se $\mathbb{Z} \cap \mathfrak{p}=p \mathbb{Z}$, onde $p$ é um número inteiro primo, então existe um único homomorfismo de anéis $\psi$ de $\mathbb{Z}[G]$ em $\mathbb{F}_{p}$, com núcleo $\mathfrak{p}$, onde $\mathbb{F}_{p}$ denota o corpo finito com $p$ elementos.

Dem.: Seja $\mathfrak{p}$ um ideal primo de $\mathbb{Z}[G]$. Para cada $g \in G$, temos que $g^{2}=1$, ou seja $0=g^{2}-1=(g-1)(g+1) \in \mathfrak{p}$. Assim $g \equiv \pm 1 \bmod \mathfrak{p}$, para todo $g \in G$ e, conseqüentemente $\frac{\mathbb{Z}[G]}{\mathfrak{p}}$ é um domínio isomorfo à $\mathbb{Z}$ ou à $\mathbb{F}_{p}$, para algum número primo $p$. Isto mostra a existência dos requeridos homomorfismos de anéis. $A$ unicidade segue do fato que $\mathbb{Z}$ e $\mathbb{Z}_{p}$ não admitirem automorfismos não triviais.

Vamos agora descrever os homomorfismos $\phi$ e $\psi$ do lema anterior. Para todo homomorfismo de anéis $\phi: \mathbb{Z}[G] \rightarrow \mathbb{Z}$ ternos que $\phi(g)= \pm 1$, para cada $g \in G$, ou seja, todo homomorfismo de anéis de $\mathbb{Z}[G]$ em $\mathbb{Z}$ leva $G$ ern $\{ \pm 1\}$. Assim a restrição $\left.\phi\right|_{G}$, de $\phi$ em $G$ é um caracter do grupo $G$, isto é, um homomorfismo de grupos $\chi$ de $G$ em $\{ \pm 1\}$. Reciprocamente, dado um caracter $\chi: G \rightarrow\{ \pm 1\}$, ele se estende, de maneira única, a um homomorfismo de anéis $\phi_{x}: \mathbb{Z}[G] \rightarrow \mathbb{Z}$, devido a propriedade universal de $\mathbb{Z}[G]$. Assim, para cada homomorfismo $\phi$ existe um único caracter $\chi$ tal que $\phi=\phi_{\chi}$.

Agora, seja $p$ um número primo ímpar. $O$ grupo $\{ \pm 1\} \subseteq \mathbb{F}_{p}$, é o subgrupo de todos os elementos de $\left(\mathbb{F}_{p}\right)^{\star}$ de ordem 2. Logo, a restrição de um hornomorfismo de anéis $\psi: \mathbb{Z}[G] \rightarrow \mathbb{F}_{p}$ ao grupo $G$, é também um caracter $\chi: G \rightarrow\{ \pm 1\}$. Assim, existe uma única extensão $\phi_{X}: \mathbb{Z}[G] \rightarrow \mathbb{Z}$ que faz o seguinte diagrama comutar

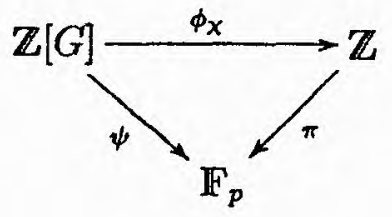


onde $\pi$ é a sobrejeção canônica de $\mathbb{Z}$ em $\mathbb{F}_{p}$.

Consideremos $p=2$. Cada homomorfismo de $\mathbb{Z}[G]$ em $\mathbb{F}_{2}$, leva todo elemento de $G$ em 1. Portanto, existe um único homomorfismo de anéis $\psi_{0}: \mathbb{Z}[G] \rightarrow \mathbb{F}_{2}$, que é obtido da composição de $\phi_{x}: \mathbb{Z}[G] \rightarrow \mathbb{Z}$ com a projeção canônica sobre $\mathbb{F}_{2}$, onde $\phi_{x}$ é a extensão de qualquer caracter $\chi: G \rightarrow\{ \pm 1\}$.

Destas observações e de (3.6), temos

Proposição 3.7 Para cada ideal primo p de $\mathbb{Z}[G]$, temos:

(i) Se $\mathbb{Z} \cap \mathfrak{p}=\{0\}$, então existe um único caracter $\chi$ de $G$, tal que $\mathfrak{p}=\mathfrak{p}_{\chi}$ é o núcleo do homomorfismo $\phi_{x}: \mathbb{Z}[G] \rightarrow \mathbb{Z} ;$

(ii) Se $\mathfrak{p} \cap \mathbb{Z}=p \mathbb{Z}, p$ um número primo ímpar, então existe um único caracter $\chi$ de $G$, tal que $\mathfrak{p}$ coincide com o conjunto

$$
\mathfrak{p}_{x, p}:=\left\{z \in \mathbb{Z}[G] ; \phi_{x}(z) \equiv 0 \bmod p\right\}
$$

(iii) Existe um único ideal $\mathfrak{p}_{0}$ de $\mathbb{Z}[G]$ com $\mathfrak{p}_{0} \cap \mathbb{Z}=2 \mathbb{Z} e$

$$
\mathfrak{p}_{0}=\left\{z \in \mathbb{Z}[G] ; \phi_{x}(z) \equiv 0 \bmod 2\right\}
$$

para cada caracter $\chi$ de $G$.

Observação 3.8 É claro que os ideais $\mathfrak{p}_{\chi}$, com $\chi$ percorrendo o conjunto dos caracteres de $G$, são todos os ideais primos minimais de $\mathbb{Z}[G]$. Os ideais $\mathfrak{p}_{\chi, p}$ com $\chi$ percorrendo o conjunto dos caracteres de $G, p$ percorrendo o conjunto dos números primos ímpares e $\mathfrak{p}_{0}$ são todos os ideais maximais de $\mathbb{Z}[G]$.

Consideremos agora, os ideais primos de $\mathcal{W}(A) \cong \frac{\mathbb{Z}[G]}{\mathcal{K}}$. Denotemos por $\mathfrak{I}(A)$ o núcleo do homomorfismo de anéis $d_{0}: \mathcal{W}(A) \rightarrow \mathbb{F}_{2}$, definido por $d_{0}([b])=(\operatorname{dim}(b)) \bmod 2$. Desde que $\frac{W(A)}{\mathfrak{I}(A)} \cong \mathbb{F}_{2}$ que é um corpo, temos que $\mathfrak{I}(A)$ é um ideal maximal de $\mathcal{W}(A)$, chamado o ideal fundamental de $\mathcal{W}(A)$.

Proposição 3.9 ideal $\mathfrak{I}(A)$ é o único ideal primo de $\mathcal{W}(A)$ que contém $2\langle 1\rangle=2.1_{\mathcal{W}(A)}$.

Dem.: Resta mostrarmos apenas a unicidade. O item (iii) da proposição anterior garante que $\mathfrak{p}_{0}$ é o único ideal primo de $\mathbb{Z}[G]$ que contém $(2) \in G$. Como $\varphi(2)=2\langle 1\rangle$, temos que 
$\mathfrak{I}(A)$ corresponde ao ideal $\mathfrak{p}_{0}$ de $\mathbb{Z}[G]$, na correspondência entre os ideais de $\mathcal{W}(A)$ e os ideais de $\mathbb{Z}[G]$ que contém $\mathcal{K}$. Portanto, a unicidade de $\mathfrak{I}(A)$ seque da unicidade de $\mathfrak{p}_{0}$.

Lema 3.10 $O$ ideal fundamental $\mathfrak{I}(A)$ é aditivamente gerado pelos elementos $\langle 1, \alpha\rangle$ em $\mathcal{W}(A), \operatorname{com} \alpha \in A^{\star}$.

Dem.: Dado $b \in \mathfrak{I}(A)$, de (3.2), podemos escrever $b=\left\langle\alpha_{1}, \ldots, \alpha_{2 n}\right\rangle$, com $\alpha_{i}$ em $A^{\star}$, $i=1, \ldots, 2 n$. Desde que $\langle 1,-1\rangle=0$ em $\mathcal{W}(A)$, temos que $b=\prod_{i=1}^{n}\left\langle 1, \alpha_{i}\right\rangle-\stackrel{1}{i=n+1}_{1}^{1}\left\langle 1,-\alpha_{i}\right\rangle$, 。 que mostra o lema.

Para uma melhor caracterização de $\operatorname{Spec}(\mathcal{W}(A))$ usaremos a noção de assinatura como definida abaixo.

Definição 3.11 Uma assinatura de $A$ é um homomorfismo de anéis de $\mathcal{W}(A)$ em $\mathbb{Z}$. Denotamos por $\mathcal{A} s s(A)$ o conjunto de todas as assinaturas de $A \mathrm{e}$, por $\mathfrak{p}_{\sigma}$ o núcleo da assinatura $\sigma$. Dizemos que $A$ é um anel formalmente real se $A$ admite pelo menos uma assinatura, isto é, se $\mathcal{A} s s(A) \neq \varnothing$. Caso contrário, $A$ é dito ser um anel não formalmente real.

Assumiremos primeiro, que $A$ é um anel formalmente real, ou seja, $\mathcal{A} s s(A) \neq \varnothing$.

Do Teorema do isomorfismo para anéis, segue imediatamente que $\frac{\mathcal{W}(A)}{\mathfrak{p}_{\sigma}} \cong \mathbb{Z}$, para toda $\sigma \in \mathcal{A} s s(A)$.

Proposição 3.12 Para cada ideal primo $\mathfrak{p}$ de $\mathcal{W}(A)$ que não contém $p\langle 1\rangle$, para todo número primo $p$, existe uma única assinatura $\sigma$ de $A$ tal que $\mathfrak{p}=\mathfrak{p}_{\sigma}$.

Dem.: Seja $\mathfrak{p}$ um ideal primo de $\mathcal{W}(A)$ que não contém $p\langle 1\rangle$, para todo número primo $p$. Desde que $\mathcal{W}(A) \cong \frac{\mathbb{Z}[G]}{\mathcal{K}}$, temos que existe $\mathfrak{p}^{\prime}=\varphi^{-1}(\mathfrak{p})$, ideal primo de $\mathbb{Z}[G]$ tal que $\mathcal{K} \subseteq \mathfrak{p}^{\prime}$. Como $p\langle 1\rangle \notin \mathfrak{p}$ para todo número primo $p$, o ideal $\mathfrak{p}^{\prime}$ é tal que $\mathfrak{p}^{\prime} \cap \mathbb{Z}=\{0\}$.

Pelo item (i) de (3.7) existe um único homomorfismo de anéis $\phi_{x}: \mathbb{Z}[G] \rightarrow \mathbb{Z}$, tal que $\operatorname{Ker}\left(\phi_{x}\right)=\mathfrak{p}^{\prime}$. Mas $\mathcal{K} \subseteq \operatorname{Ker}\left(\phi_{x}\right)=\mathfrak{p}^{\prime}$. Logo, existe uma única $\sigma \in \mathcal{A} s s(A)$ que faz o seguinte diagrama comutar

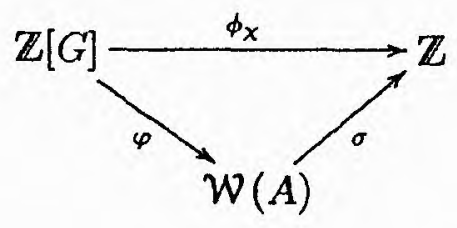


Ainda falta mostrarmos que $\mathfrak{p}=\mathfrak{p}_{\sigma}$. Desde que $\sigma(\mathfrak{p})=\sigma \circ \varphi\left(\mathfrak{p}^{\prime}\right)=\phi_{\chi}\left(\mathfrak{p}^{\prime}\right)=0$, temos que $\mathfrak{p} \subseteq \mathfrak{p}_{\sigma}$. Por outro lado, dado $b \in \mathfrak{p}_{\sigma}$, o fato de $\varphi$ ser sobrejetora implica que existe $x \in \mathbb{Z}[G]$ tal que $\varphi(x)=b$. Assim, $\phi_{x}(x)=\sigma \circ \varphi(x)=\sigma(b)=0$, ou seja $x \in \operatorname{Ker}\left(\phi_{x}\right)=\mathfrak{p}^{\prime}$. Logo, $\varphi(x)=b \in \mathfrak{p}$. Portanto $\mathfrak{p}_{\sigma} \subseteq \mathfrak{p}$, como queríamos.

Para analisarmos os ideais primos de $\mathcal{W}(A)$ que contém $p\langle 1\rangle$, para algum primo ímpar $p$, precisamos das informaçôes sobre o ideal $\mathcal{X}$ contidas no seguinte lema.

Lema 3.13 Seja z um elemento de $\mathbb{Z}[G]$ de uma das seguintes formas:

(i) $z=\sum_{i=1}^{r}\left(\alpha_{i}\right)-\sum_{i=1}^{r}\left(\beta_{i}\right), \operatorname{com}\left\langle\alpha_{1}, \ldots, \alpha_{r}\right\rangle \simeq\left\langle\beta_{1}, \ldots \beta_{r}\right\rangle$ e $r \leq 5$;

(ii) $z=(1)+(-1)$.

Então, para qualquer caracter $\chi$ de $G$, temos $\phi_{\chi}(z)=0$ ou $\pm 2^{n}$ para algum inteiro $n \geq 1$.

Dem.: A conclusão é clara para (ii). Portanto, suponha $z$ como em (i). Então $\prod_{i=1}^{r}\left(\alpha_{i}\right)=\prod_{i=1}^{r}\left(\beta_{i}\right)$ em $G=A^{\star} /\left(A^{\star}\right)^{2}$. Agora fixemos $\chi$ e suponha $s$ o número de elementos $\left(\alpha_{i}\right)$ onde $\chi\left(\alpha_{i}\right)=-1, \log 0 r-s$ é o número de elementos $\left(\alpha_{i}\right) \operatorname{com} \chi\left(\alpha_{i}\right)=1$. Então $\sum_{i=1}^{r} \chi\left(\alpha_{i}\right)=r-2 s$. Agora $\prod_{i=1}^{r} \chi\left(\alpha_{i}\right)=1$ se, e somente se $s$ é par, o que é equivalente a $\sum_{i=1}^{r} \chi\left(\alpha_{i}\right) \equiv r \bmod 4$. Mais ainda, $\prod_{i=1}^{r} \chi\left(\alpha_{i}\right)=-1$ se, e somente se $s$ é impar, o que é equivalente a $\sum_{i=1}^{r} \chi\left(\alpha_{i}\right) \equiv(r-2) \bmod 4$. Assim, segue em ambos os casos que

$$
\sum_{i=1}^{r} \chi\left(\alpha_{i}\right) \equiv \sum_{i=1}^{r} \chi\left(\beta_{i}\right) \bmod 4
$$

Logo $\phi_{x}(z) \equiv 0 \bmod 4$. Mas $r \leq 5$ implicando que $\phi_{x}(z)$ é um inteiro com valor absoluto $\leq 10$. Portanto $\phi_{\chi}(z)=0, \pm 4$ ou \pm 8 e o resultado segue.

Corolário 3.14 Para cada caracter $\chi$ de $G$, temos $\phi_{\chi}(\mathcal{K})=0$ ou $\phi_{\chi}(\mathcal{K})=2^{n} \mathbb{Z}$, para algum $n \geq 1$.

Dem.: Segue diretamente de (3.4) e (3.13). 
Corolário 3.15 Se $\phi_{\chi}(\mathcal{K}) \subseteq p \mathbb{Z}$, para algum primo ímpar $p$, então $\phi_{\chi}(\mathcal{K})=0$.

Dem.: Imediata.

Proposição 3.16 Seja $p$ um número primo ímpar. Para cada ideal primo p de $\mathcal{W}(A)$ com $p\langle 1\rangle \in \mathfrak{p}$, existe uma única assinatura $\sigma$ de $A$ tal que $\mathfrak{p}$ coincide com o conjunto

$$
\mathfrak{p}_{\sigma, p}=\{b \in \mathcal{W}(A) ; \sigma(b) \equiv 0 \bmod p\}
$$

Dem.: Seja $\mathfrak{p}$ um ideal primo de $\mathcal{W}(A) \operatorname{com} p\langle 1\rangle \in \mathfrak{p}$. Novamente pelo isomorfismo $\mathcal{W}(A) \cong \frac{\mathbb{Z}[G]}{\mathcal{K}}$ temos que existe $\mathfrak{p}^{\prime} \subseteq \mathbb{Z}[G]$ tal que $\mathcal{X} \subseteq \mathfrak{p}^{\prime}$ e $\varphi\left(\mathfrak{p}^{\prime}\right)=\mathfrak{p}$. Como $p\langle 1\rangle \in \mathfrak{p}$ temos que $\mathfrak{p}^{\prime} \cap \mathbb{Z}=p \mathbb{Z}$. Por (3.7), temos que existe um único caracter $\chi$ de $G$, tal que $\mathfrak{p}^{\prime}=\mathfrak{p}_{\chi, p}=\left\{z \in \mathbb{Z}[G] ; \phi_{\chi}(z) \equiv 0 \bmod p\right\}$.

Do fato que $\mathcal{K} \subseteq \mathfrak{p}^{\prime}=\mathfrak{p}_{\chi, p}$, segue que $\phi_{\chi}(\mathcal{K}) \subseteq p \mathbb{Z}$. Assim de (3.15) temos que $\phi_{x}(\mathcal{K})=0$. Portanto $\mathcal{K} \subseteq K e r\left(\phi_{x}\right)$ e, conseqüentemente, existe uma única $\sigma \in \mathcal{A} s s(A)$ que faz o diagrama abaixo comutar

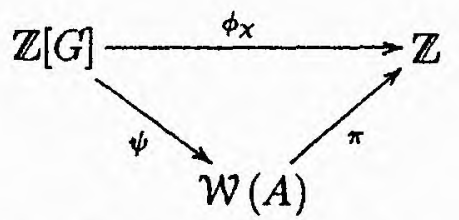

Mostremos agora que $\mathfrak{p}=\mathfrak{p}_{\sigma, p}$. Dado $b \in \mathfrak{p}$, existe $x \in \mathfrak{p}^{\prime}$ tal que $\varphi(x)=b$, pois $\mathfrak{p}^{\prime}=\varphi^{-1}(\mathfrak{p})$. Da definição de $\mathfrak{p}^{\prime}$, temos que $\phi_{x}(x) \equiv 0 \bmod p$. Assim, $\sigma(b)=\sigma \circ \varphi(x)=$ $\phi_{x}(x) \equiv 0 \bmod p$, ou seja, $b \in \mathfrak{p}_{\sigma, p}$, mostrando que $\mathfrak{p} \subseteq \mathfrak{p}_{\sigma, p}$. Seja agora $b \in \mathfrak{p}_{\sigma, p}$. Segue da sobrejetividade de $\varphi$ que existe $x \in \mathbb{Z}[G]$ tal que $\varphi(x)=b$. Assim, $\phi_{x}(x)=\sigma \circ \varphi(x)=$ $\sigma(b) \equiv 0 \bmod p$, isto é, $x \in \mathfrak{p}^{\prime} . \log , b=\varphi(x) \in \mathfrak{p}$. Portanto, $\mathfrak{p}_{\sigma, p}=\mathfrak{p}$, como queríamos.

De (3.7) e da correspondência entre os ideais de $\mathcal{W}(A)$ com os ideais de $\mathbb{Z}[G]$, que contém $\mathcal{X}$, concluimos que se $\mathcal{A s s}(A) \neq \varnothing$ então $\mathfrak{p}_{\sigma}, \mathfrak{p}_{\sigma, p}$, para cada $\sigma \in \mathcal{A} s s(A)$ e todo número primo ímpar $p$, e $\mathfrak{I}(A)$ são todos os ideais primos de $\mathcal{W}(A)$.

Teorema 3.17 Se A é um anel semilocal formalmente real, então:

(i) Os $\mathfrak{p}_{\sigma}$, com $\sigma \in \mathcal{A} s s(A)$, são todos os ideais primos minimais de $\mathcal{W}(A)$; 
(ii) Os $\mathfrak{p}_{\sigma, p}$ com $\sigma \in \mathcal{A} s s(A)$ e $p$ um número primo ímpar, e $\mathfrak{I}(A)$ são todos os ideais primos maximais de $\mathcal{W}(A)$;

(iii) Cada $\mathfrak{p}_{\sigma, p}$ contém um único ideal primo minimal, a saber $\mathfrak{p}_{\sigma}$;

(iv) $\mathfrak{I}(A)$ contém todos os ideais primos minimais.

Dem.: Resta apenas mostrarmos (iii) e (iv). Em $\mathbb{Z}$ existe um único ideal maximal que contém $p$. Então, devido ao isomorfismo $\mathcal{W}(A) / \mathfrak{p}_{\sigma} \cong \mathbb{Z}$, existe um único ideal maximal de $\mathcal{W}(A)$ que contém $p\langle 1\rangle$ e $\mathfrak{p}_{\sigma}$, que é claramente $\mathfrak{p}_{\sigma, p}$. Suponhamos que exista um ideal primo minimal $\mathfrak{p}_{\psi}$ de $\mathcal{W}(A)$, tal que $\mathfrak{p}_{\psi}$ também está contido em $\mathfrak{p}_{\sigma, p}$. Como $\mathfrak{p}_{\sigma, p}$ contém $\mathfrak{p}_{\psi}$ e $p\langle 1\rangle$ temos que $\mathfrak{p}_{\sigma, p}=\mathfrak{p}_{\psi, p}$. Segue então de (3.16) que $\sigma=\psi$. Portanto $\mathfrak{p}_{\sigma}=\mathfrak{p}_{\psi}$, o que mostra (iii).

Agora, queremos mostrar que $\mathfrak{p}_{\sigma} \subseteq \mathfrak{I}(A)$, para cada $\sigma \in \mathcal{A} s s(A)$. Para isto, seja $b=\left\langle\alpha_{1}, \ldots, \alpha_{n}\right\rangle \in \mathfrak{p}_{\sigma}$. Como $\sigma\left(\left\langle\alpha_{i}\right\rangle\right)= \pm 1$, para $1 \leq i \leq n$ e, $\sigma(b)=0$, temos que $n$ tem de ser par e para metade dos índices $i=1, \ldots, n, \sigma\left(\left\langle\alpha_{i}\right\rangle\right)=1$, e para a outra metade $\sigma\left(\left\langle\alpha_{i}\right\rangle\right)=-1$. Portanto $\mathfrak{p}_{\sigma} \subseteq \mathfrak{I}(A)$, como queríamos.

Para o caso em que $A$ é um anel não formalmente real temos:

Teorema $3.18 O$ anel $A$ é não formalmente real se, e somente se $\mathfrak{I}(A)$ é o único ideal primo de $\mathcal{W}(A)$.

Dem.: Da descrição dos ideais de $\mathbb{Z}[G]$, temos claramente que se $\mathcal{A} s s(A)=\varnothing$, então $\mathfrak{I}(A)$ é o único ideal primo de $\mathcal{W}(A)$. Reciprocamente se $\mathfrak{I}(A)$ é o único ideal primo de $\mathcal{W}(A)$, então $\mathfrak{I}(A)$ é o nilradical de $\mathcal{W}(A)$, isto é, o conjunto de todos os elementos nilpotentes de $\mathcal{W}(A)$. Em particular, $2\langle 1\rangle \in \mathfrak{I}(A)$ é nilpotente. Assim existe $n \geq 1$ tal que $2^{n}\langle 1\rangle=(2\langle 1\rangle)^{n}=0$ em $\mathcal{W}(A)$, implicando que $\langle 1\rangle \in \mathcal{W}(A)$ é um elemento de torção. Como $\mathbb{Z}$ é um anel livre de torção e assumimos que todo homomorfismo de anéis leva elemento identidade em elemento identidade, temos que não existe homomorfismo de anéis de $\mathcal{W}(A)$ em $\mathbb{Z}$, ou seja, $A$ é não formalmente

real.

Como uma consequência imediata deste teorema temos: 
Corolário 3.19 Se A é não formalmente real, enlão os divisores de zero de $\mathcal{W}(A)$ tem dimensão par.

Dem.: De (3.18) temos que $\mathcal{W}(A)$ é um anel local com um único ideal maximal $\mathfrak{I}(A)$. Portanto, os divisores de zero, que são elementos não invertíveis de $\mathcal{W}(A)$, estào em $\mathfrak{I}(A)$.

\subsection{Nilpotência e Torção}

Nesta seção apresentamos alguns resultados sobre os elementos de torção, os elementos nilpotentes e os divisores de zero do anel de Witt de $A$.

Desde que $\mathcal{W}(A)$ é um anel comutativo com elemento identidade $\langle 1\rangle$, o conjunto dos elementos nilpotentes de $\mathcal{W}(A)$ formam o nilradical de $\mathcal{W}(A)$, que denotaremos por $\mathcal{N i l}(\mathcal{W}(A))$. 0 ideal dos elementos de torção de $\mathcal{W}(A)$ será denotado por $\mathcal{W}(A)_{T}$.

Em um anel $R$, um elemento de torção $x \in R$ é dito ter $p$-torção, $p \in \mathbb{Z}$ um número primo, se $x$ é anulado por uma potência de $p$. Dentre os resultados que apresentaremos nesta seção, mostraremos que o anel $\mathcal{W}(A)$ tem somente 2-torção.

Começaremos assumindo $A$ é um anel semilocal não formalmente real. Neste caso, como consequência imediata de (3.18) e sua demonstração, temos:

Teorema 3.20 Se A é um anel semilocal não formalmente real, então:

(i) $\mathcal{N i l}(\mathcal{W}(A))=\mathfrak{I}(A)$;

(ii) $\mathcal{W}(A)_{T}=\mathcal{W}(A)$;

(iii) $\mathcal{W}(A)$ tem somente 2-torção.

Dem.: Imediata.

Agora seja $A$ um anel semilocal formalmente real. Desde que os $\mathfrak{p}_{\sigma} \operatorname{com} \sigma \mathrm{em} A s s(A)$, são todos os ideais primos minimais de $\mathcal{W}(A)$, temos:

Proposição 3.21 Um elemento $b \in \mathcal{W}(A)$ é nilpotente se, e somente se $\sigma(b)=0$ para toda $\sigma \in \mathcal{A} s s(A)$, isto é, $\mathcal{N i l}(\mathcal{W}(A))=\bigcap_{\sigma \in \mathcal{A} s s(A)} \operatorname{Ker}(\sigma)$. 
Dem.: Imediata.

Para o ideal de torção temos:

Proposição 3.22 Se A é um anel semilocal formalmente real, então

$$
\mathcal{W}(A)_{T}=\mathcal{N i l}(\mathcal{W}(A))
$$

Dem.: Sejam $b \in \mathcal{W}(A)_{T}$ e $n \in \mathbb{N}, n \geq 1$, tal que $n b=0$. Então, para cada $\sigma$ em Ass $(A)$, temos $\sigma(n b)=n \sigma(b)=0$, o que implica que $\sigma(b)=0$. Assim, $b$ pertence a $\bigcap_{\sigma \in \mathcal{A} s s(A)} \operatorname{Ker}(\sigma)=\mathcal{N}$ il $(\mathcal{W}(A))$, o que mostra que $\mathcal{W}(A)_{T} \subseteq \mathcal{N i l}(\mathcal{W}(A))$. Reciprocamente, dado $b=\left\langle\alpha_{1}, \ldots, \alpha_{n}\right\rangle \in \mathcal{N i l}(\mathcal{W}(A))$; consideremos $H$ o subgrupo de $G$ gerado por $\left\{\left(\alpha_{1}\right), \ldots,\left(\alpha_{n}\right)\right\}$. Então, desde que todo elemento de $G$ tem ordem 2, temos que $H$ é um subgrupo finito de $G$ e $b$ está no subanel $R$ de $\mathcal{W}(A)$ isomorfo á $\frac{\mathbb{Z}[H]}{(\mathcal{K} \cap \mathbb{Z}[H])}$. Usando o teorema de Maschke (cf. (3.6) em [Pie]) temos que o anel de grupo $\mathbf{Q}[H] \cong \mathbf{Q} \otimes \mathbb{Z}[H]$ é semi-simples, ou seja, $\mathcal{N} i l(\mathbb{Q}[H])=\{0\}$.

Desde que $R$ é imagem homomórfica de $\mathbb{Z}[H]$, temos que $\mathbb{Q} \otimes R$ é imagem homomórfica de $\mathbf{Q} \otimes \mathbb{Z}[H]$ e por (3.1(b) de [Pie]) segue que $\mathcal{N i l}(\mathbf{Q} \otimes R)=\{0\}$.

Mas, $1 \otimes b \in \mathbb{Q} \otimes \mathcal{N i l}(R) \subseteq \mathcal{N i l}(\mathbb{Q} \otimes R)=\{0\}$. Também identificando $\mathbb{Q} \otimes R$ com $\mathrm{T}^{-1}(\mathbb{Z}) \otimes R \cong \mathrm{T}^{-1}(R)$, onde $\mathrm{T}=\mathbb{Z}-\{0\}$, temos $0=1 \otimes b=\frac{b}{1} \in \mathrm{T}^{-1}(R)$, o que é equivalente a existir $n \in \mathrm{T}, n \geq 1$, tal que $n b=0$ em $R$. Assim, $b \in R_{T} \subseteq \mathcal{W}(A)_{T}$, o que mostra a proposição.

Das duas últimas proposições, deduzimos imediatamente o Princípio Local-Global de Pfister para espaços bilineares sobre um anel semilocal formalmente real.

Teorema 3.23 (Princípio Local-Global de Pfister) Seja $A$ um anel semilocal formalmente real. Então uma forma bilinear $b$ representa um elemento de torção $\mathrm{em} \mathcal{W}(A)$ se, $e$ somente se $\sigma(b)=0$ para toda $\sigma \in \mathcal{A s s}(A)$.

Dem.: Imediata.

Mostremos agora que também no caso em que $A$ é formalmente real $\mathcal{W}(A)$ também tem somente 2-torção. 
Teorema 3.24 Se A é um anel semilocal formalmente real, então $\mathcal{W}(A)$ tem somente 2-torção.

Dem.: Seja $b=\left\langle\alpha_{1}, \ldots, \alpha_{n}\right\rangle \in \mathcal{W}(A)_{T}$. Desde que $\mathcal{W}(A)_{T}=\mathcal{N}$ il $(\mathcal{W}(A))$, temos que $b \in \mathcal{N} i l(\mathcal{W}(A))$ e, como na demonstração da proposição anterior, $b$ está no subanel $R$ de $\mathcal{W}(A)$ isomorfo à $\frac{\mathbb{Z}[H]}{(\mathcal{K} \cap \mathbb{Z}[H])}$, onde $H$ é o subgrupo de $G$ gerado por $\left\{\left(\alpha_{1}\right), \ldots,\left(\alpha_{n}\right)\right\}$. Agora, para mostrarmos que $b$ é 2-torção em $\mathcal{W}(A)$, é suficiente mostrarmos que o anel $R$ não tem p-torção, para todo número primo ímpar $p$.

Seja $p$ um número primo impar qualquer. Desde que $R \cong \frac{\mathbb{Z}[H]}{(\mathcal{K} \cap \mathbb{Z}[H])}$, temos que $\frac{R}{p R} \cong \frac{\mathbb{F}_{p}[H]}{\mathcal{K}^{\prime}}$, para algum subgrupo $\mathcal{K}^{\prime}$ de $\mathbb{F}_{p}[H]$.

Como $H \subseteq G$, temos que os elementos de $H$ tem ordem 2. Desde que $\mathbb{F}_{p}$ tem característica $p$ um primo ímpar, novamente pelo teorema (3.6) de Maschke [Pie], obtemos que $R / p R$ é um anel semi-simples, isto é, $\mathcal{N} i l(R / p R)=\{0\}$.

Se $b_{0} \in R$ é nilpotente, então $\overline{b_{0}} \in \mathcal{N} i l(R / p R)=\{0\}$, ou seja, existe $b_{1} \in R$ tal que $b_{0}=p b_{1}$. Isto, e o fato que $\mathcal{W}(A)_{T}=\mathcal{N} i l(\mathcal{W}(A))$, mostra que o ideal de torção $R_{T}$ de $R$ é divisível por cada número primo ímpar, isto é, $R_{T}=p R_{T}$ para cada número primo ímpar p.

Se $b_{0} \in R$ tem $p$-torção, então $b_{0} \in R_{T}=p R_{T}$, ou seja, $b_{0}=p b_{1}$, com $b_{1}$ em $R_{T}$ que também tem $p$-torção, o que implica em $b_{1}=p b_{2}$, para algum $b_{2} \in R_{T}$ e conseqüentemente, $b_{0}=p^{2} b_{2}$. Assim, para cada inteiro $n \geq 0$, existe $b_{n} \in R_{T}$, onde $b_{n}$ tem $p$-torção e $b_{0}=p^{n} b_{n}$.

Mas $R$ é um grupo abeliano finitamente gerado, então pela decomposição dos $\mathbb{Z}$-módulos finitamente gerados, temos que $R_{T}$ é um grupo finito. Portanto, existe $N>0$ tal que $p^{N} b^{\prime}=0$, para todo $b^{\prime} \in R_{T}$ com p-torção. Logo $b_{0}=p^{N} b_{N}=0$ em $R$. Como $p$ é um número primo ímpar qualquer, temos que $R$ não tem $p$-torção, para todo número primo ímpar $p$, como queríamos.

Como consequência do teorema anterior deduzimos que, também no caso em que $A$ é formalmente real, os divisores de zero de $\mathcal{W}(A)$ tem dimensão par.

Corolário 3.25 Os divisores de zero de $\mathcal{W}(A)$ tem dimensão par.

Dem.: Em [Kap], página 3, temos que o conjunto dos divisores de zero de $\mathcal{W}(A)$ é uma união de ideais primos. Suponhamos que $p\langle 1\rangle$ é um divisor de zero, para algum número 
primo impar $p$. Então existe $b \in \mathcal{W}(A), b \neq 0$, tal que $p\langle 1\rangle \otimes b=0$ em $\mathcal{W}(A)$, implicando que $b$ tem $p$-torção em $\mathcal{W}(A)$. Mas, do teorema anterior temos que $\mathcal{W}(A)$ tem somente. 2-torção. Assim, $p\langle 1\rangle$ não é divisor de zero para nenhum primo ímpar $p$. Assim na união dos ideais primos que compoem os divisores de zero, não aparecem os ideais primos da forma $\mathfrak{p}_{\sigma, p}$, com $\sigma \in \mathcal{A} s s(A)$ e $p$ um número primo ímpar, ou seja de (3.17), temos que cada ideal primo que aparece na união é minimal ou $\mathfrak{I}(A)$. Desde que $\mathfrak{p}_{\sigma} \subseteq \mathfrak{I}(A)$, para todo $\sigma \in \mathcal{A s s}(A)$, o resultado segue.

Finalizamos esta seção, apresentando um resultado que nos seá útil no final do trabalho. Considere o módulo quadrático $\left(E_{8}, q\right)$ sobre $A$, cuja matriz da forma bilinear associada é

$$
b_{q}=\left(\begin{array}{cccccccc}
2 & 0 & -1 & 0 & 0 & 0 & 0 & 0 \\
0 & 2 & 0 & -1 & 0 & 0 & 0 & 0 \\
-1 & 0 & 2 & -1 & 0 & 0 & 0 & 0 \\
0 & -1 & -1 & 2 & -1 & 0 & 0 & 0 \\
0 & 0 & 0 & -1 & 2 & -1 & 0 & 0 \\
0 & 0 & 0 & 0 & -1 & 2 & -1 & 0 \\
0 & 0 & 0 & 0 & 0 & -1 & 2 & -1 \\
0 & 0 & 0 & 0 & 0 & 0 & -1 & 2
\end{array}\right) .
$$

De [Ser], temos que $E_{8}$ é de fato um espaço quadrático e que $b_{q} \simeq 8\langle 1\rangle$.

Proposição 3.26 Se $q^{\prime} \in \mathcal{W}_{Q}(A)$ é tal que $b_{q^{\prime}}=0$ em $\mathcal{W}(A)$, então $8 q^{\prime}=0$ em $\mathcal{W}_{Q}(A)$.

Dem.: Seja $\left(E_{8}, q\right)$ o espaço quadrático citado acima. Desde que $b_{q^{\prime}}=0$ em $\mathcal{W}(A)$, temos que

$$
b_{q^{\prime}} \otimes q=0 \quad \text { em } \quad \mathcal{W}_{Q}(A) .
$$

Assim $0=b_{q^{\prime}} \otimes q=q^{\prime} \odot q=q^{\prime} \otimes b_{q}=b_{q} \otimes q^{\prime}=8\langle 1\rangle \otimes q^{\prime}=8 q^{\prime}$, como queríamos.

\subsection{Resultados sobre Assinaturas}

Finalizaremos este capítulo apresentando alguns conceitos e resultados envolvendo assinaturas e espaços bilineares e/ou quadráticos que serão usados no próximo capítulo. Nesta seção, assumiremos que $A$ é um anel semilocal formalmente real. 
$\operatorname{Sejam}(M, b) \in \mathcal{B i l}(A)$ e $\sigma \in \mathcal{A} s s(A)$. Denotamos por $\sigma(b)$ a imagem por $\sigma$ do elemento $b \in \mathcal{W}(A)$. É fácil ver que $|\sigma(b)| \leq \operatorname{dim}(b)$ e $|\sigma(b)| \equiv \operatorname{dim}(b) \bmod 2$, onde $|\sigma(b)|$ é o valor absoluto do número inteiro $\sigma(b)$.

Diremos que o espaço bilinear $(M, b)$, ou a forma bilinear $b$ é definido com relação a assinatura $\sigma$, se $|\sigma(b)|=\operatorname{dim}(b)$. Além disso, dizemos que $(M, b)$ é positivo definido, respectivamente negativo definido com relação à $\sigma$ se $\sigma(b)=\operatorname{dim}(b)$, respectivamente $\sigma(b)=-\operatorname{dim}(b)$. Dizemos que $(M, b)$ é indefinido com relação à $\sigma$ se $|\sigma(b)|<\operatorname{dim}(b)$.

Seja $(E, q) \in Q_{u a d}(A)$. Para qualquer $\sigma \in \mathcal{A} s s(A)$, escrevemos $\sigma(q)$ para denotar $\sigma\left(b_{q}\right)$ e, dizemos que $(E, q)$ é um espaço quadrático definido, positivo definido, negativo definido ou indefinido com relação à $\sigma$ se o espaço bilinear associado $\left(E, b_{q}\right)$ possuir tal propriedade.

Dado $(M, b) \in \mathcal{B} i l(A)$, respectivamente $(E, q) \in \operatorname{Quad}(A)$, dizemos que o elemento $\alpha \in A^{*}$ é representado por $b$, respectivamente por $q$, se existe $x \in M$ tal que $b(x, x)=\alpha$, respectivamente $q(x)=\alpha$.

Lema 3.27 Sejam $\sigma \in \mathcal{A s s}(A)$ e $(M, b)$ um espaço bilinear próprio sobre $A$. Se $(M, b)$ é positivo definido em relação à $\sigma$, então $\sigma(\langle\beta\rangle)=1$, para cada unidade $\beta$ de $A$ representado por $b$.

Dem.: De (2.21) temos que existem $\alpha_{1}, \ldots, \alpha_{n} \in A^{*}$ tais que $b \simeq\left\langle\alpha_{1}, \ldots, \alpha_{n}\right\rangle$. Se $\beta \in A^{*}$ é representado por $b$, então de (2.16), (ii), temos que existe um subespaço $N$ de $M$ tal que $M \simeq A x \perp N$ com $b(x, x)=\beta$. Como $(N, b)$ pode ser impróprio, somando $\langle 1\rangle$ em ambos os lados temos que existem $\beta_{1}, \ldots, \beta_{n} \in A^{*}$ tais que

$$
\left\langle\alpha_{1}, \ldots, \alpha_{n}, 1\right\rangle \simeq\langle\beta\rangle \perp b_{\mid N} \perp\langle 1\rangle \simeq\left\langle\beta, \beta_{1}, \ldots, \beta_{n}\right\rangle
$$

Desde que $(M, b)$ é positivo definido, temos que $n=\operatorname{dim}(b)=\sigma(b)=\sum_{i=1}^{n} \sigma\left(\left\langle\alpha_{i}\right\rangle\right)$, ou seja, $\sigma\left(\left\langle\alpha_{i}\right\rangle\right)=1$, para todo $i=1 \ldots, n$. Assim $n+1=\sigma(b)+1=\sigma(b \perp(1\rangle)=$ $\sigma\left(\left\langle\beta, \beta_{1}, \ldots, \beta_{n}\right\rangle\right)=\sigma(\langle\beta\rangle)+\sum_{i=1}^{n} \sigma\left(\left\langle\beta_{i}\right\rangle\right)$. Como $\operatorname{Im}(\sigma) \subseteq \mathbb{Z}$, temos que isso ocorre somente se $\sigma(\langle\beta\rangle)=\sigma\left(\left\langle\beta_{1}\right\rangle\right)=\ldots=\sigma\left(\left\langle\beta_{n}\right\rangle\right)=1$, como queríamos.

Lema 3.28 Seja $(M, q)$ um espaço quadrático positivo definido em relação à $\sigma$. Então $\sigma(\langle\gamma\rangle)=1$ para cada $\gamma \in A^{*}$ representado por $M$.

Dem.: Suponhamos que existe $\lambda \in A^{*}$ tal que $\sigma(\langle\gamma\rangle)=-1$. Desde que $(M, q)$ é positivo definido em relação à $\sigma$, temos que $\sigma\left(b_{q} \perp\langle-\gamma\rangle\right)=\operatorname{dim}\left(b_{q}\right)+1$, ou seja $b_{q} \perp(-\gamma\rangle$ é um 
espaço bilinear positivo definido em relação à $\sigma$. Desde que $b_{q}(x, x)=2 q(x)$, para todo $x \in M$ e $q$ representa $\gamma$, temos que $b_{q}$ representa $2 \gamma$, o que implica que $\gamma=2 \gamma-\gamma$ é uma unidade representada pelo espaço bilinear $b_{q} \perp\langle-\gamma\rangle$ positivo definido em relação à $\sigma$ com $\sigma(\langle\gamma\rangle)=-1$, o que contradiz o lema anterior. Logo $\sigma(\langle\gamma\rangle)=1$ para todo $\gamma \in A^{\star}$ representado por $q$.

Corolário 3.29 Seja $(M, q)$ um espaço quadrático negativo definido em relação à $\sigma$ em $\mathcal{A} s s(A)$. Então $\sigma(\langle\gamma\rangle)=-1$, para toda unidade $\gamma$ de A representada por $q$.

Dem.: Do lema anterior é suficiente mostrarmos que se $(M, q)$ é negativo definido em relação à $\sigma$, então $(M,-q)$ é positivo definido em relação à $\sigma$ e representa $-\gamma$. Para tanto, basta observarmos que $-q \simeq\langle-1\rangle \otimes q$.

Sejam $h \geq 1$ um número inteiro tal que $(2 h-1)(4 h-1) \in A^{\star}$, que existe por (1.6) e $(M, q) \circ$ espaço quadrático do exemplo (2.4), ou seja $M=A x \oplus A y$, e $q=[1, h]$.

Lema 3.30 Para cada $\sigma \in \mathcal{A s s}(A)$, temos que $\sigma([1, h])=2$.

Dem.: Seja $(M, q)$ o espaço quadrático descrito acima. Queremos mostrar que $\sigma\left(b_{q}\right)=2$, para cada $\sigma \in \mathcal{A} s s(A)$. Considere o espaço bilinear $\left(M^{\prime}, b\right)$, onde $M^{\prime}=A x \oplus A y \oplus A z \mathrm{e}$ $b=b_{q} \perp\langle-1\rangle$ que tem a seguinte matriz em relação a base $\{x, y, z\}$

$$
\left(\begin{array}{ccc}
2 & 1 & 0 \\
1 & 2 h & 0 \\
0 & 0 & -1
\end{array}\right) \text {. }
$$

Os elementos $x^{\prime}=x+z$ e $y^{\prime}=y+z$ geram um subespaço não singular de $\left(M^{\prime}, b\right)$, onde $b_{\mid N}=\langle 1,2 h-1\rangle$. De (2.26), temos que existe $z^{\prime} \in M^{\prime}$ tal que $b=b_{q} \perp\langle-1\rangle \simeq$ $\langle 1,2 h-1\rangle \perp\left\langle b\left(z^{\prime}, z^{\prime}\right)\right\rangle$. Comparando os determinantes temos que podemos tomar $z^{\prime} \in M^{\prime}$ $\operatorname{com} b\left(z^{\prime}, z^{\prime}\right)=(2 h-1)(1-4 h)$. Assim temos:

$$
b_{q} \perp\langle-1\rangle \simeq\langle 1,2 h-1,(2 h-1)(1-4 h)\rangle .
$$

Desde que todo inteiro positivo pode ser escrito com soma de 4 quadrados, temos que existem $c_{1}, c_{2}, c_{3}, c_{4} \in A$ tais que

$$
4 h-1=c_{1}^{2}+c_{2}^{2}+c_{3}^{2}+c_{4}^{2},
$$


ou seja, $4 h-1$ é uma unidade em $A$ representada pelo espaço bilinear $\langle 1,1,1,1\rangle$ que é positivo definido em relação à $\sigma$, para cada $\sigma \in \mathcal{A s s}(A)$. Logo, de $(\mathbf{3 . 2 7}), \sigma(\langle 4 h-1\rangle)=1$, o que implica que $\sigma(\langle 1-4 h\rangle)=-1$. Assim

$$
\sigma\left(b_{q} \perp\langle-1\rangle\right)=1+\sigma(\langle 2 h-1\rangle)(1+\sigma(\langle 1-4 h\rangle))=1
$$

ou seja, $\sigma\left(b_{q}\right)=2$ como queríamos. 


\section{Capítulo 4}

\section{Equivalências Topológicas do Espaço}

\section{das Assinaturas}

Alex Rosenberg e Roger Ware mostraram em [RW] que a Propriedade da Aproximação Forte, a Propriedade da Aproximação Fraca e a Propriedade de Hasse-Minkowiski, que apresentaremos neste capítulo, são equivalentes para o espaço topológico das assinaturas Ass $(A)$, onde $A$ é um anel semilocal com $2 \in A^{\star}$. Neste capítulo apresentaremos um estudo da Topologia de Zariski em $\mathcal{A s s}(A)$ e, usando as idéias de Manfred Knebush em [Kne1], mostraremos as mesmas equivalências obtidas em [RW] sem requerer 2 como uma unidade no anel $A$.

No que segue, $A$ será sempre um anel semilocal formalmente real, $G=A /\left(A^{\star}\right)^{2}$ o grupo das classes quadradas das unidades de $A$ e $\mathcal{W}(A) \cong \mathbb{Z}[G] / \mathcal{K}$ o anel de Witt dos espaços bilineares sobre $A$, conforme descrito no capítulo anterior.

\subsection{O espaço das assinaturas $\mathcal{A} s s(A)$}

Nosso objetivo, nesta seção, é definir uma topologia no conjunto $\mathcal{A s s}(A)$, onde $A$ é um anel semilocal formalmente real, e apresentar algumas características básicas do espaço das assinaturas de $A, \mathcal{A} s s(A)$.

De (3.5) temos que $W(A)$ é gerado por subespaços de dimensão 1 e, desde que toda assinatura de $A$ é uma extensão de algum caracter de $G$, podemos considerar que $\mathcal{A} s s(A)$ é um subconjunto do conjunto de todas as funções de $G$ a valores em $\{ \pm 1\}$. 
Este conjunto de funções pode ser visto como o produto direto $\prod_{G}\{ \pm 1\}$. Munindo $\{ \pm 1\}$ com a topologia discreta e $\prod_{G}\{ \pm 1\}$ com a topologia produto, temos do Teorema de Tychonoff (cf. [Munkres]), que $\prod_{G}\{ \pm 1\}$ é um espaço topológico compacto. Assim, $\mathcal{A} s s(A)$, com a topologia induzida, é um subespaço topológico de $\prod_{G}\{ \pm 1\}$.

Dado $\alpha \in G$, seja $\pi_{\alpha}: \prod_{G}\{ \pm 1\} \rightarrow\{ \pm 1\}$ a projeção na $\alpha$-ésima coordenada.

Para $\alpha \in G$ e $\varepsilon= \pm 1$, seja $H(\alpha, \varepsilon)=\{f: G \rightarrow\{ \pm 1\} ; f(\alpha)=\varepsilon\}$. É fácil ver que os $H(\alpha, \varepsilon)=\pi_{\alpha}^{-1}(\varepsilon)$, com $\alpha \in G$ e $\varepsilon \in\{ \pm 1\}$, formam uma subbase para a topologia produto em $\prod_{G}\{ \pm 1\}$. Ainda, podemos escrever $\prod_{G}\{ \pm 1\}=H(\alpha, \varepsilon) \cup H(\alpha,-\varepsilon)$, onde a união é disjunta, o que mostra que para cada $\alpha \in G$ e $\varepsilon \in\{ \pm 1\}$, os conjuntos $H(\alpha, \varepsilon)$ são fechabertos e, $\prod_{G}\{ \pm 1\}$ é um espaço topológico totalmente desconexo.

Este espaço é também um espaço de Hausdorff pois se, $f, g \in \prod_{G}\{ \pm 1\}$ com $f \neq g$, então existe $\alpha \in G$ tal que $f(\alpha) \neq g(\alpha)$ e, conseqüentemente $f \in H(\alpha, \varepsilon)$ e $g \in H(\alpha,-\varepsilon)$, onde $\varepsilon \in\{ \pm 1\}$ é tal que $f(\alpha)=\varepsilon$.

Para o subespaço $\mathcal{A} s s(A)$ do espaço topológico $\prod_{G}\{ \pm 1\}$, temos:

Teorema 4.1 Se A é um anel semilocal formalmente real, então $\mathcal{A s s}(A)$ é um espaço topológico compacto, de Hausdorff e totalmente desconexo.

Dem.: Como as propriedades de Hausdorff e totalmente desconexo são herdadas da topologia induzida, é suficiente mostrarmos que $\mathcal{A s s}(A)$ é compacto. Desde que $\prod_{G}\{ \pm 1\}$ é compacto, basta mostrarmos que $\mathcal{A s s}(A)$ é um subespaço fechado de $\prod_{G}\{ \pm 1\}$, o que é equivalente a mostrarmos que o seu complementar $Y=\prod_{G}\{ \pm 1\}-\mathcal{A s s}(A)$ é aberto.

Seja $f: G \rightarrow\{ \pm 1\}$ um elemento de $Y$. Desde que $\mathcal{W}(A)$ é gerado por $\{\langle\alpha\rangle ; \alpha \in G\}$, temos que $f$ não é um homomorfismo de grupos, pois caso contrário, $f$ admitiria uma extensão para uma assinatura de $A$, o que contradiz o fato de $f \in Y$. Assim, existem $\alpha$, $\beta \in G$ tais que $f(\alpha \beta) \neq f(\alpha) f(\beta)$. Se $f(\alpha \beta)=-1$, então $f(\alpha)=-f(\beta)=\varepsilon, \operatorname{com} \varepsilon=\{ \pm 1\}$ e $H(\alpha, \varepsilon) \cap H(\beta, \varepsilon) \cap H(\alpha \beta,-1)$ é um aberto que contém $f$ que está contido em $Y$.

Se $f(\alpha \beta)=1$, então $f(\alpha)=f(\beta)=\varepsilon, \operatorname{com} \varepsilon=\{ \pm 1\}$ e, neste caso, $H(\alpha, \varepsilon) \cap H(\alpha, \varepsilon) \cap$ $H(\alpha \beta, 1)$ é um aberto contido em $Y$ que contém $f$. Logo $Y$ é um subconjunto aberto de 
$\prod_{G}\{ \pm 1\}$, como queríamos.

Usando a subbase $\{H(\alpha, \varepsilon) ; \alpha \in G, \varepsilon \in\{ \pm 1\}\}$ de $\prod_{G}\{ \pm 1\}$, obtemos uma subbase para a topologia de $\mathcal{A s s}(A)$ dada pelos conjuntos

$$
\{H(\alpha, \varepsilon) ; \alpha \in G, \varepsilon \in\{ \pm 1\}\} \cap \mathcal{A s s}(A) .
$$

Para $\alpha \in G$ temos

$$
\begin{aligned}
H(\alpha,-1) \cap \mathcal{A} s s(A) & =\left\{f \in \prod_{G}\{ \pm 1\} ; f(\alpha)=-1\right\} \cap \mathcal{A} s s(A) \\
& =\{\sigma \in \mathcal{A} s s(A) ; \sigma(\langle\alpha\rangle)=-1\} \\
& =\{\sigma \in \mathcal{A} s s(A) ; \sigma(\langle-\alpha\rangle)=1\} \\
& =\left\{f \in \prod_{G}\{ \pm 1\} ; f(-\alpha)=1\right\} \cap \mathcal{A} s s(A) \\
& =H(-\alpha, 1) \cap \mathcal{A} s s(A) .
\end{aligned}
$$

Assim, podemos dizer que $\{H(\alpha) ; \alpha \in G\}$, onde $H(\alpha)=H(\alpha, 1) \cap \mathcal{A s s}(A)$, forma uma subbase de $\mathcal{A s s}(A)$, chamada subbase de Harrison. Também chamaremos de Topologia de Harrison de $\mathcal{A s s}(A)$ a topologia gerada por esta subbase. Lembremos que $H(\alpha)$ é fechaberto para cada $\alpha \in G$, mais ainda, podemos escrever

$$
H(\alpha)=\{\sigma \in \mathcal{A s s}(A) ; \sigma(\langle\alpha\rangle)=1\}
$$

para cada $\alpha \in A^{\star}$. Note que, sem perda de generalidade, podemos trocar $H(\alpha)$ por $W(\alpha)=\{\sigma \in \mathcal{A} s s(A) ; \sigma(\langle\alpha\rangle)=-1\}$, para cada $\alpha \in A^{\star}$, pois $W(\alpha)=H(-\alpha)$.

O próximo teorema relaciona a topologia de Harrison de $\mathcal{A} s s(A)$ com a topologia de Zariski induzida em $\operatorname{Smin}(\mathcal{W}(A))$, o conjunto dos ideais primos minimais de $\mathcal{W}(A)$. Uma vez estabelecido este resultado quando nos referirmos a topologia de $\mathcal{A s s}(A)$, estaremos falando da topologia gerada pela seguinte coleção de subconjuntos $W(\alpha)=$ $\{\sigma \in \mathcal{A} s s(A) ; \sigma(\langle\alpha\rangle)=-1\}, \alpha \in A^{\star}$, que formam uma subbase de fechabertos da topologia de Harrison-Zariski em $\mathcal{A s s}(A)$.

Teorema 4.2 $O$ espaço topológico Ass $(A)$, com a topologia de Harrison é homeomorfo ao espaço topológico $\operatorname{Smin}(\mathcal{W}(A))$, com a topologia de Zariski induzida. 
Dem.: De (3.17),(i), temos que a função $\Phi: \mathcal{A s s}(A) \rightarrow \operatorname{Smin}(A)$, definida por $\Phi(\sigma)=$ $\mathfrak{p}_{\sigma}=\operatorname{Ker}(\sigma)$, é uma bijeção. Agora mostremos que $\Phi$ é de fato um homeomorfismo entre os espaços topológicos $\mathcal{A s s}(A)$ e $\operatorname{Smin}(A)$.

Primeiramente observemos que a topologia de Zariski induzida em $S \min (A)$ possui como base a coleção $\{U(b) \cap \operatorname{Smin}(\mathcal{W}(A)) ; b \in \mathcal{W}(A)\}$, onde $U(b)=\{\mathfrak{p} \in \operatorname{Spec}(\mathcal{W}(A)) ; b \notin \mathfrak{p}\}$ é um aberto básico da topologia de Zariski em $\operatorname{Spec}(\mathcal{W}(A))$. De (3.17),(iii), podemos refinar esta coleção para $\{U(b) \cap \operatorname{Smin}(\mathcal{W}(A)) ; b \in \mathfrak{I}(A)\}$. Agora seja $U(b) \cap \operatorname{Smin}(\mathcal{W}(A))$ um aberto básico de $\operatorname{Smin}(\mathcal{W}(A))$. Desde que $b \in \mathfrak{I}(A)$, de (3.10) temos que $b=\prod_{i=1}^{n}\left\langle 1, \alpha_{i}\right\rangle$, $\alpha_{i} \in A^{*}, 1 \leq i \leq n$. Uma vez que $U\left(\underset{i=1}{\perp}\left\langle 1, \alpha_{i}\right\rangle\right)=\bigcup_{i=1}^{n} U\left(\left\langle 1, \alpha_{i}\right\rangle\right)$, é suficiente considerarmos $b=\langle 1, \alpha\rangle, \operatorname{com} \alpha \in A^{\star}$. Neste caso

$$
\begin{aligned}
\Phi^{-1}(U(b) \cap \operatorname{Smin}(\mathcal{W}(A))) & =\Phi^{-1}(U(\langle 1, \alpha\rangle) \cap \operatorname{Smin}(\mathcal{W}(A))) \\
& =\{\sigma \in \mathcal{A} s s(A) ; \sigma(\langle 1, \alpha\rangle) \neq 0\} \\
& =\{\sigma \in \mathcal{A s s}(A) ; \sigma(\sigma(\langle\alpha\rangle)=1\}=W(-\alpha) .
\end{aligned}
$$

Logo $\Phi$ é contínua.

Se $\Psi=\Phi^{-1}: \operatorname{Smin}(\mathcal{W}(A)) \rightarrow \mathcal{A s s}(A)$, é a inversa de $\Phi$, então $\Psi$ é definida por $\mathfrak{p}_{\sigma}=$ $\operatorname{Ker}(\sigma) \mapsto \sigma$, para cada $\mathfrak{p}_{\sigma} \in \operatorname{Smin}(\mathcal{W}(A))$. Para $\alpha \in A^{\star}$, seja $W(\alpha)=$ $\{\sigma \in \mathcal{A} s s(A) ; \sigma(\langle\alpha\rangle)=-1\}=\{\sigma \in \mathcal{A} s s(A) ; \sigma(\langle 1,-\alpha\rangle) \neq 0\}$ um aberto básico da topologia de $\mathcal{A s s}(A)$. Então

$$
\begin{aligned}
\Psi^{-1}(W(\alpha)) & =\Psi^{-1}(\{\sigma \in \mathcal{A s s}(A) ; \sigma(\langle 1,-\alpha\rangle) \neq 0\}) \\
& =\{\mathfrak{p} \in \operatorname{Smin}(\mathcal{W}(A)) ;\langle 1,-\alpha\rangle \notin \mathfrak{p}\} \\
& =U(\langle 1,-\alpha\rangle) \cap \operatorname{Smin}(\mathcal{W}(A)) .
\end{aligned}
$$

Logo $\Psi$ é contínua e o resultado segue.

\subsection{Propriedades Topológicas}

Nesta seção apresentaremos algumas propriedades topológicas do espaço das assinaturas de $A$.

Considere o espaço $\mathcal{A s s}(A)$ com a topologia de Harrison-Zariski. Com relação a subbase $\left\{W(\alpha) ; \alpha \in A^{\star}\right\}$, definimos as seguintes propriedades: 
$\left(\mathbf{P}_{1}\right)$ - (Propriedade da Aproximação Fraca) - O conjunto $\left\{W(\alpha) ; \alpha \in A^{\star}\right\}$ forma uma base para a topologia de Harrison-Zariski de $\mathcal{A} s s(A)$.

$\left(\mathbf{P}_{2}\right)$ - (Propriedade da Aproximação Forte) - Cada fechaberto de $\mathcal{A s s}(A)$ tem a forma $W(\alpha)$, para algum $\alpha \in A^{*}$.

$\left(\mathbf{P}_{3}\right)$ - Para cada conjunto de unidades $\alpha_{1}, \ldots, \alpha_{m} \in A^{\star}$, existe $\alpha \in A^{\star}$ tal que $W\left(\alpha_{1}\right) \cap \ldots \cap W\left(\alpha_{m}\right)=W(\alpha)$.

Com relação aos elementos de $\mathcal{A} s s(A)$, temos as seguintes propriedades topológicas:

$\left(\mathbf{P}_{4}\right)$ - Se $Y_{1}, Y_{2}$ são dois subconjuntos fechados disjuntos de $\mathcal{A} s s(A)$, então existe um elemento $\alpha$ em $A^{\star} \operatorname{com} \sigma(\langle\alpha\rangle)=1$ para todo $\sigma \in Y_{1}$, e $\tau(\langle\alpha\rangle)=-1$ para todo $\tau \in Y_{2}$.

$\left(\mathbf{P}_{\mathbf{5}}\right)$ - Para qualquer subconjunto fechado $Y \operatorname{de} \mathcal{A} s s(A)$ e qualquer $\tau \in \mathcal{A} s s(A)-Y$, existe um elemento $\alpha \in A^{\star} \operatorname{com} \sigma(\langle\alpha\rangle)=1$ para todo $\sigma \in Y$ e $\tau(\langle\alpha\rangle)=-1$.

$\left(\mathbf{P}_{\mathbf{6}}\right)$ - (Propriedade de Hasse-Minkowiski) - Para cada espaço bilinear $(M, b)$ satisfazendo $|\sigma(b)|<\operatorname{dim}(b)$, para toda assinatura $\sigma \in \mathcal{A s s}(A)$, existe um inteiro positivo $m$ tal que $m b$ é isotrópico.

Listemos agora algumas relações imediatas entre estas propriedades.

É imediato que $\left(\mathbf{P}_{\mathbf{2}}\right)$ implica $\left(\mathbf{P}_{\mathbf{1}}\right)$. Na próxima seção mostraremos que estas propriedades são de fato equivalentes. Aqui observemos que $\left(\mathbf{P}_{\mathbf{1}}\right)$ e $\left(\mathbf{P}_{\mathbf{2}}\right)$ são consequiência de $\left(\mathbf{P}_{\mathbf{3}}\right)$.

Proposição 4.3 As propriedades $\left(\mathbf{P}_{1}\right)$ e $\left(\mathbf{P}_{2}\right)$ são conseqüência da propriedade $\left(\mathbf{P}_{3}\right)$.

Dem.: É imediato que $\left(\mathbf{P}_{1}\right)$ segue de $\left(\mathbf{P}_{3}\right)$. Mostremos que $\left(\mathbf{P}_{\mathbf{2}}\right)$ também segue de $\left(\mathbf{P}_{3}\right)$. Seja $Y$ um conjunto fechaberto de $\mathcal{A s s}(A)$. Desde que $\mathcal{A} s s(A)$ é compacto e $\mathcal{A} s s(A)-Y$ é fechado, temos que $\mathcal{A} s s(A)-Y$ é também compacto.

Como $\left(\mathbf{P}_{3}\right)$ implica $\left(\mathbf{P}_{1}\right)$, temos que existem $\alpha_{1}, \ldots, \alpha_{n} \in A^{\star}$ tais que

$$
\mathcal{A} s s(A)-Y=\bigcup_{i=1}^{n} W\left(-\alpha_{i}\right) \text {. }
$$

Uma vez que $\mathcal{A} s s(A)-W(-\alpha)=W(\alpha)$, obtemos

$$
Y=\bigcap_{i=1}^{n}\left(\mathcal{A} s s(A)-W\left(-\alpha_{i}\right)\right)=\bigcap_{i=1}^{n} W\left(\alpha_{i}\right)=W(\alpha),
$$


para algum $\alpha$ em $A^{\star}$, implicando en $\left(\mathbf{P}_{2}\right)$.

O próximo resultado será útil nas proximas seções:

Lema 4.4 Seja $(M, b) \in \mathcal{B} i l(A)$ próprio. A aplicação $f_{b}: \mathcal{A s s}(A) \rightarrow \mathbb{Z}$, definida por $f(\sigma)=\sigma(b)$ para toda $\sigma \in \mathcal{A} s s(A)$, é contínua.

Dem.: Naturalmente estamos considerando a topologia discreta em $\mathbb{Z}$. Desde que a soma. de funções contínuas é contínua, podemos assumir de (3.5) que $b$ é unidimensional. Para. $b=\langle\alpha\rangle, \alpha \in A^{\star}$, temos:

$$
\begin{aligned}
& f_{b}^{-1}(i)=\{\sigma \in \mathcal{A} s s(A) ; \sigma(\langle\alpha\rangle)=i\}=\varnothing \text { se } i \neq \pm 1 \\
& f_{b}^{-1}(1)=\{\sigma \in \mathcal{A} s s(A) ; \sigma(\langle\alpha\rangle)=1\}=W(-\alpha) \\
& f_{b}^{-1}(-1)=\{\sigma \in \mathcal{A} s s(A) ; \sigma(\langle\alpha\rangle)=-1\}=W(\alpha) .
\end{aligned}
$$

Uma vez que estes conjuntos são abertos em $\mathcal{A s s}(A)$, nosso lema segue.

\subsection{Equivalências}

Para concluirmos nosso trabalho, nesta seção, apresentaremos as demonstrações necessárias para mostrarmos que as seis propriedades topológicas do espaço das assinaturas de $A$, apresentadas na seção anterior, são propriedades equivalentes.

Teorema 4.5 As propriedades $\left(\mathbf{P}_{2}\right)$ e $\left(\mathbf{P}_{4}\right)$ são equivalentes.

Dem.: Suponha que $\mathcal{A}_{s s}(A)$ satisfaz $\left(\mathbf{P}_{2}\right)$. Sejam $Y_{1}, Y_{2}$ subconjuntos fechados disjuntos $\operatorname{de} \mathcal{A} s s(A)$. De (1.14), Ass $(A)$ é um espaço topológico normal, logo existe um subconjunto aberto $X$ de $\mathcal{A s s}(A)$ tal que $Y_{1} \subseteq X$ e $Y_{2} \cap X=\varnothing$. Desde que $\left(\mathbf{P}_{2}\right)$ implica $\left(\mathbf{P}_{I}\right)$, temos que $X$ é uma união de subconjuntos fechabertos da forma $W(\beta), \operatorname{com} \beta \in A^{\star}$. Agora, como $Y_{1} \subseteq X$ e $Y_{1}$ é compacto, existem $\alpha_{1}, \ldots, \alpha_{n} \in A^{\star}$ tais que $W\left(\alpha_{i}\right)$ aparece na união que forma $X$ e $Y_{1} \subseteq \bigcup_{i=1}^{n} W\left(\alpha_{i}\right)$

Como $\bigcup_{i=1}^{n} W\left(\alpha_{i}\right)$ é um fechaberto de $\mathcal{A} s s(A)$, temos de $\left(\mathbf{P}_{2}\right)$ que existe $\alpha \in A^{\star}$ tal que
$\bigcup_{i=1}^{n} W\left(\alpha_{i}\right)=W(\alpha)$ e, conseqüentemente, $Y_{1} \subseteq W(\alpha) \subseteq X$ e $Y_{2} \cap W(\alpha)=\varnothing$. Com isso 
temos que existe $\alpha \in A^{\star}$ tal que $\sigma(\langle\alpha\rangle)=-1$, para toda $\sigma \in Y_{1}$ e $\tau(\langle\alpha\rangle)=1$, para toda $\tau \in Y_{2}$, implicando em $\left(\mathbf{P}_{4}\right)$.

Reciprocamente, suponha que $\mathcal{A s s}(A)$ satisfaz $\left(\mathbf{P}_{\mathbf{4}}\right)$ e considere $Y$ um subconjunto fechaberto de $\mathcal{A s s}(A)$. Então $\mathcal{A s s}(A)-Y$ é um subconjunto fechaberto e claramente disjunto de $Y$. Por $\left(\mathbf{P}_{4}\right)$ existe $\alpha \in A^{\star} \operatorname{com} \sigma(\langle\alpha\rangle)=-1$ para toda $\sigma \in Y$ e $\tau(\langle\alpha\rangle)=1$ para toda. $\tau \in \mathcal{A s s}(A), \log \mathrm{Y}=W(\alpha)$.

Teorema 4.6 As propriedades $\left(\mathbf{P}_{1}\right)$ e $\left(\mathbf{P}_{2}\right)$ são equivalentes, ou seja, os conjuntos $W(\alpha)$ formam uma base para a topologia de $\mathcal{A} s s(A)$ se, e somente se cada fachaberto de $\mathcal{A} s s(A)$ é da forma $W(\alpha)$, para algum $\alpha \in A^{\star}$.

Dem.: Como observado na seção anterior, $\left(\mathbf{P}_{\mathbf{2}}\right)$ implica $\left(\mathbf{P}_{1}\right)$. Assim, é suficiente mostrarmos que a propriedade $\left(\mathbf{P}_{2}\right)$ é conseqüência da propriedade $\left(\mathbf{P}_{1}\right)$. Mas, de (4.3), basta mostrarmos que $\left(\mathbf{P}_{\mathbf{1}}\right)$ implica $\left(\mathbf{P}_{\mathbf{3}}\right)$.

Suponhamos então que o espaço topológico $\mathcal{A s s}(A)$ satisfaz a propriedade $\left(\mathbf{P}_{1}\right)$. Sejam $\alpha_{1}, \ldots, \alpha_{n} \in A^{\star}$ e $Y=\bigcap_{i=1}^{n} W\left(\alpha_{i}\right)$. Queremos mostrar que existe $\alpha \in A^{*}$ tal que $Y=W(\alpha)$. Desde que cada $W\left(\alpha_{i}\right)$ é um fechaberto em $\mathcal{A s s}(A)$, temos que $Y$ é também um fechaberto em Ass $(A)$, o que implica que $Y$ é um aberto compacto. Assim, de $\left(\mathbf{P}_{2}\right)$, temos que existem $\beta_{1}, \ldots, \beta_{k} \in A^{\star}$ tais que $Y=W\left(\beta_{1}\right) \cup \ldots \cup W\left(\beta_{k}\right)$. Repetindo alguns abertos na interseção ou na união, se necessário, podemos supor que $n=k$, ou seja

$$
Y=\bigcap_{i=i}^{n} W\left(\alpha_{i}\right)=\bigcup_{i=1}^{n} W\left(\beta_{i}\right)
$$

onde $\alpha_{i}, \beta_{i} \in A^{\star}$, para $1 \leq i \leq n$.

Considere os espaços bilineares sobre $A,(M, b)$ e $\left(N, b^{\prime}\right)$, onde

$$
b=\otimes_{i=1}^{n}\left\langle 1,-\alpha_{i}\right\rangle \text { e } b^{\prime}=\otimes_{i=1}^{n}\left\langle 1, \beta_{i}\right\rangle \text {. }
$$

Desde que para cada $\sigma \in Y, \sigma\left(\left\langle\alpha_{i}\right\rangle\right)=-1$, para todo $i=1, \ldots, n$ e $\sigma\left(\left\langle\beta_{j}\right\rangle\right)=-1$, para algum $j=1, \ldots, n$, temos que

$$
\sigma(b)=\prod_{i=1}^{n} \sigma\left(\left\langle 1,-\alpha_{i}\right\rangle\right)=\prod_{i=1}^{n}\left(1+\sigma\left(\left\langle-\alpha_{i}\right\rangle\right)\right)=2^{n}=\operatorname{dim}(b)
$$

e

$$
\sigma\left(b^{\prime}\right)=\prod_{i=1}^{n} \sigma\left(\left\langle 1, \beta_{i}\right\rangle\right)=\prod_{i=1}^{n}\left(1+\sigma\left(\left\langle\beta_{i}\right\rangle\right)\right)=0 .
$$


De maneita análoga obtemos que para cada $\tau \in \mathcal{A s s}(A)-Y, \tau(b)=0$ e $\tau\left(b^{\prime}\right)=2^{n}=$ $\operatorname{dim}\left(b^{\prime}\right)$. Portanto para cada $\sigma \in \mathcal{A} s s(A)$ temos que $\sigma\left(b \perp b^{\prime}\right)=2^{n}$, o que implica que $\sigma\left(b \perp b^{\prime} \perp 2^{n}\langle-1\rangle\right)=0$, para toda $\sigma \in \mathcal{A s s}(A)$. Assim, de (3.23) o elemento $b \perp b^{\prime} \perp 2^{n}\langle-1\rangle$ em $\mathcal{W}(A)$ é de torção. De (3.24), temos que existe um inteiro $m \geq 0$ tal que $2^{m}\left(b \perp b^{\prime} \perp 2^{n}(-1)\right)=0$ em $\mathcal{W}(A)$, ou seja

$$
2^{m} b \perp 2^{m} b^{\prime}=2^{m+n}\langle 1\rangle \text { em } \mathcal{W}(A)
$$

Sejam $h \geq 1$ um inteiro, $\operatorname{com}(1-2 h)(1-4 h) \in A^{\star}$ e $q=[1, h]$, como em (2.4). Tensorizando a igualdade acima por $q$, obtemos

$$
2^{m}(b \otimes q) \perp 2^{m}\left(b^{\prime} \otimes q\right)=2^{m+n} q \quad \text { em } \quad W_{Q}(A) .
$$

Usando que vale o Teorema do Cancelamento de Witt para espaços quadráticos (cf. (2.35)), a definição de $W_{Q}(A)$ e que $\operatorname{dim}(b \otimes q)=\operatorname{dim}\left(b^{\prime} \otimes q\right)=2^{n+1}$, obtemos a isometria de espaços quadráticos

$$
2^{m}(b \otimes q) \perp 2^{m}\left(b^{\prime} \otimes q\right) \simeq 2^{m+n} q \perp 2^{m+n} \mathrm{H}
$$

onde H é o plano hiperbólico. Assim $2^{m}(b \otimes q) \perp 2^{m}\left(b^{\prime} \otimes q\right)$ é um espaço quadrático isotrópico sobre $A$. De [Ba], Theorem (5.2), existe $\alpha \in A^{\star}$ tal que $\alpha$ é representado por $2^{m}(b \otimes q)$ e $-\alpha$ é representado por $2^{m}\left(b^{\prime} \otimes q\right)$. Agora, dado $\sigma \in Y$, desde que $\sigma(q)=2$ (cf. (3.30)), temos que $\sigma\left(2^{m}(b \otimes q)\right)=2^{m+1} \sigma(b)=2^{m+n+1}=\operatorname{dim}\left(2^{m}(b \otimes q)\right)$. Logo $2^{m}(b \otimes q)$ é um espaço quadrátivo positivo definido com relação a $\sigma \in Y$ e, como $\alpha$ é representado por este espaço, de (3.28) ternos que $\sigma(\langle\alpha\rangle)=1$, para todo $\sigma \in Y$. De maneira análoga mostra-se que $2^{m}\left(b^{\prime} \otimes q\right)$ é um espaço quadrático positivo definido em relação a cada $r \in \mathcal{A} s s(A)-Y$ e, portanto, $\tau(\langle-\alpha\rangle)=1$, ou seja $\tau(\langle\alpha\rangle)=-1$. Conseqüentemente $Y=W(\alpha)$, isto é, $W\left(\alpha_{1}\right) \cap \ldots \cap W\left(\alpha_{n}\right)=W(\alpha)$, o que mostra que o espaço topológico $\mathcal{A s s}(A)$ satisfaz a propriedade $\left(\mathbf{P}_{3}\right)$, como queríamos.

Teorema 4.7 As propriedades $\left(\mathbf{P}_{1}\right)$ e $\left(\mathbf{P}_{5}\right)$ são equivalentes.

Dem.: Assuma que $\mathcal{A s s}(A)$ satisfaz $\left(\mathbf{P}_{1}\right)$. Sejam $Y$ um subconjunto fechaclo de $\mathcal{A s s}(A)$, e $\tau \in \mathcal{A s s}(A)-Y$. Desde que $\mathcal{A s s}(A)$ é compacto e $Y$ é fechado, temos que existem $\alpha_{1}, \ldots, \alpha_{n} \in A^{\star}$ tais que $Y=\bigcup_{i=1}^{n} W\left(\alpha_{i}\right)$. De (4.5), Ass $(A)-Y=\bigcap_{i=1}^{n} W\left(-\alpha_{i}\right)=W(\alpha)$, 
para algum $\alpha \in A^{\star}$. Isto implica que $\tau(\langle-\alpha\rangle)=1$. Mais ainda, como $Y=\mathcal{A s s}(A)-W(\alpha)=$ $W(-\alpha)$, temos que $\sigma(\langle\alpha\rangle)=1$ para toda $\sigma \in Y$, implicando em $\left(\mathbf{P}_{5}\right)$.

Reciprocamente, assuma que $\mathcal{A s s}(A)$ satisfaz $\left(\mathbf{P}_{5}\right)$. De (4.3) é suficiente mostrarmos que Ass $(A)$ satisfaz $\left(\mathbf{P}_{3}\right)$. Seja $Y=W\left(\alpha_{1}\right) \cap \ldots \cap W\left(\alpha_{n}\right)$, com $\alpha_{i} \in A^{*}, i=1, \ldots, n$. Como $Y$ é fechado, temos que existe $\alpha \in A^{*}$ tal que $\sigma(\langle\alpha\rangle)=1$ para toda $\sigma \in Y$ e $r(\langle\alpha\rangle)=-1$ para toda $\tau \in \mathcal{A s s}(A)-Y$, por $\left(\mathbf{P}_{5}\right)$. Assim $Y=W(-\alpha)$ e o resultado segue.

Se $(M, b)$ è um espaço bilinear com $b=\otimes_{i=1}^{n}\left\langle 1, \alpha_{i}\right\rangle=\left\langle\left\langle\alpha_{1}, \ldots, \alpha_{n}\right\rangle\right\rangle$, com $\alpha_{i} \in A^{\star}$, $1 \leq i \leq n$, então dizemos que $(M, b)$ é um $n$-espaço de Pfister, ou simplesmente, que $b$ é uma $n$-forma de Pfister.

Dois $n$-espaços de Pfister $(M, b)$ e $\left(M^{\prime}, b^{\prime}\right)$ são ditos estarem conectados se existir um $(n-1)$-espaço de Pfister $\left(E, b^{\prime \prime}\right)$ e unidades $\alpha, \beta \in A$ tais que

$$
b \simeq\langle 1, \alpha\rangle \otimes b^{\prime \prime} \quad \text { e } \quad b^{\prime} \simeq\langle 1, \beta\rangle \otimes b^{\prime \prime}
$$

Os espaços $b$ e $b^{\prime}$ são ditos estarem estavelmente conectados se existir um inteiro $m \geq 1$ tal que $2^{m} b$ e $2^{m} b^{\prime}$ estão conectados.

Com esta noção temos as seguintes equivalências:

Teorema 4.8 Para um anel semilocal $A$, as seguintes afirmações são equivalentes:

(i) Ass (A) satisfaz $\left(\mathbf{P}_{1}\right)$;

(ii) Ass (A) satisfaz $\left(\mathbf{P}_{2}\right)$;

(iii) Para cada $n$-espaço bilinear de Pfister b, existe um número inteiro positivo $m$ e um elemento $\alpha \in A^{\star}$ tal que $2^{m} b \simeq 2^{m+n-1}\langle 1, \alpha\rangle$;

(iv) Cada n-espaço bilinear de Pfister está estávelmente conectado ao espaço $2^{n}\langle 1\rangle$;

(v) Quaisquer dois n-espaços bilineares de Pfister estão estávelmente conectados.

Dem.: A equivalência das afirmações $(i)$ e (ii) é (4.6).

Suponha que $\mathcal{A s s}(A)$ satisfaz $\left(\mathbf{P}_{2}\right)$. Seja $b=\left\langle\left\langle\alpha_{1}, \ldots, \alpha_{n}\right\rangle\right\rangle$ um $n$-espaço bilinear de Pfister. Para $Y=W\left(\alpha_{1}\right) \cup \ldots \cup W\left(\alpha_{n}\right)$, temos que $\sigma(b)=0$ para toda $\sigma \in Y$ e $\tau(b)=2^{n}$, para toda $r \in \mathcal{A s s}(A)-Y$. Desde que cada $W\left(\alpha_{i}\right)$ é um fechaberto de $\mathcal{A s s}(A)$, temos que $Y$ é um fechaberto de $\mathcal{A}_{s s}(A)$. Logo, existe $\alpha \in A^{\star}$ tal que $Y=W(\alpha)$. Agora é fácil ver 
que $\sigma(b)=\sigma\left(2^{n-1}\langle 1, \alpha\rangle\right)$, para todo $\sigma \in \mathcal{A} s s(A)$, ou seja $b \perp\left(2^{n-1}\langle-1,-\alpha\rangle\right)$ é um elemento de torção de $\mathcal{W}(A)$. Repetindo o mesmo racioncínio utilizado na demonstração do teorema (4.6), obtemos um inteiro positivo $m$ tal que $2^{m} b \simeq 2^{m+n-1}\langle 1, \alpha\rangle$, o que mostra (iii).

Se $A$ satisfaz a condição (iii), temos que existe um inteiro $m$ e $\alpha \in A^{\star}$ tais que

$$
2^{m} b \simeq 2^{m+n-1}\langle 1, \alpha\rangle \simeq\langle 1, \alpha\rangle \otimes b^{\prime}
$$

onde $b^{\prime}=\otimes_{i=1}^{m+n-1}\langle 1,1\rangle \simeq 2^{m+n-1}\langle 1\rangle$. Agora,

$$
2^{m+n}\langle 1\rangle \simeq \otimes_{i=1}^{m+n}\langle 1,1\rangle=\langle 1,1\rangle \otimes b^{\prime}
$$

o que mostra que $A$ satisfaz a condição (iv).

Assuma que $A$ satisfaz a condição (iv). Sejam $b$ e $b^{\prime}$ dois $n$-espaços bilineares de Pfister sobre $A$. Por hipótese, existem inteiros positivos $m, m^{\prime}$, unidades $\alpha, \beta, \gamma, \delta$ em $A, b_{1}$ um $(m+n-1)$-espaço bilinear de Pfister e $b_{2}$ e um $\left(m^{\prime}+n-1\right)$-espaço bilinear de Pfister tais que

$$
\begin{array}{ll}
2^{m} b \simeq\langle 1, \alpha\rangle \otimes b_{1}, & 2^{m+n}\langle 1\rangle \simeq\langle 1, \beta\rangle \otimes b_{1}, \\
2^{m^{\prime}} b^{\prime} \simeq\langle 1, \gamma\rangle \otimes b_{2}, & 2^{m^{\prime}+n}\langle 1\rangle \simeq\langle 1, \delta\rangle \otimes b_{2} .
\end{array}
$$

Dado $\sigma \in \mathcal{A s s}(A)$, temos que $\sigma\left(2^{m+n}\langle 1\rangle\right)=2^{m+n}$ e, como $2^{m+n}\langle 1\rangle=\langle 1, \beta\rangle \otimes b_{1}$, temos que $2^{m+n}=\sigma\left(\langle 1, \beta\rangle \otimes b_{1}\right)=(1+\sigma(\langle\beta\rangle)) \sigma\left(b_{1}\right)$. Logo $\sigma(\langle\beta\rangle)=1$, para toda $\sigma \in \mathcal{A} s s(A)$, ou seja, $b_{1} \perp 2^{m+n-1}\langle-1\rangle \in \mathcal{W}_{T}(A)$. Repetindo o raciocínio usado na demonstração do teorema (4.6), e usando que $\operatorname{dim}\left(b_{1}\right)=2^{m+n-1}$, obtemos que existe um inteiro $k \geq 0$ tal que $2^{k} b_{1} \simeq 2^{m+n+k-1}\langle 1\rangle$. De maneira análoga obtemos que existe um inteiro $k^{\prime} \geq 0$ tal que $2^{k^{\prime}} b_{2} \simeq 2^{m^{\prime}+n+k^{\prime}-1}\langle 1\rangle$. Destas isometrias e das. 4 isometrias listadas acima, obtemos que

$$
\begin{aligned}
& 2^{m+k+m^{\prime}+k^{\prime}} b \simeq\langle 1, \alpha\rangle \otimes 2^{m+k+m^{\prime}+k^{\prime}-1}\langle 1\rangle, \\
& 2^{m+k+m^{\prime}+k^{\prime}} b^{\prime} \simeq\langle 1, \gamma\rangle \otimes 2^{m+k+m^{\prime}+k^{\prime}-1}\langle 1\rangle,
\end{aligned}
$$

ou seja, $b$ e $b^{\prime}$ estão estavelmente conectados, o que mostra $(v)$.

Finalmente, assuma que $A$ satisfaz $(v)$. Para mostrarmos que $\mathcal{A s s}(A)$ satisfaz $\left(\mathbf{P}_{1}\right)$, de (4.3) é suficiente mostrarmos que $\mathcal{A s s}(A)$ satisfaz $\left(\mathbf{P}_{3}\right)$. Para isto sejam $\alpha_{1}, \ldots, \alpha_{n}$ em $A^{*}$ tais que $Y=\bigcap_{i=1}^{n} W\left(\alpha_{i}\right)$. Considerando os $n$-espaços de Pfister $b=\left\langle\left\langle-\alpha_{1}, \ldots,-\alpha_{n}\right\rangle\right\rangle$ e $b^{\prime}=2^{n}\langle 1\rangle$, temos de $(v)$ que existe um inteiro positivo $m$, um $(n+m-1)$-espaço de Pfister $b_{1}$, e unidades $\alpha, \beta \in A^{\star}$ satisfazendo

$$
2^{m} b \simeq\langle 1, \alpha\rangle \otimes b_{1}, \quad 2^{m} b^{\prime}=2^{m+n}\langle 1\rangle \simeq\langle 1, \beta\rangle \otimes b_{1}
$$


Da segunda isometria obtemos que $\sigma\left(b_{1}\right)=2^{m+n-1}=\operatorname{dim}\left(b_{1}\right)$, para toda $\sigma$ em $\mathcal{A} s s(A)$. Da primeira isometria temos $(1+\sigma(\langle\alpha\rangle)) 2^{m+n-1}=2^{m} \sigma(b)$, para toda $\sigma$ em $\mathcal{A} s s(A)$. Desde que

$$
\sigma(b)=\left\{\begin{array}{lll}
2^{n}, & \text { se } & \sigma \in Y \\
0, & \text { se } & \sigma \notin Y
\end{array}\right.
$$

temos que

$$
\sigma(\langle\alpha\rangle)=\left\{\begin{array}{ccc}
1, & \text { se } & \sigma \in Y \\
-1, & \text { se } & \sigma \notin Y
\end{array}\right.
$$

o que mostra que $Y=W(\alpha)$, completando assim a demonstração do teorema.

Para finalizar, basta mostrarmos a equivalência entre as propriedades $\left(\mathbf{P}_{\mathbf{2}}\right)$ e $\left(\mathbf{P}_{\mathbf{6}}\right)$. Mostremos primeiramente a seguinte equivalência.

Teorema 4.9 As seguintes afirmações são equivalentes para um anel semilocal $A$ :

$\left(\mathbf{P}_{2}\right)$ - Cada fechaberto do espaço Ass $(A)$ é da forma $W(\alpha)$, para algum $\alpha \in A^{\star}$;

$\left(\mathbf{P}_{\mathbf{o}}^{\prime}\right)$ - Se um espaço quadrático $(M, q)$ sobre $A$ é indefinido para todas as assinaturas de $A$, então existe um número inteiro $m \geq 0$ tal que $m q$ é uma forma quadrática isotrópica.

Dem.: Considere que $A$ satisfaz $\left(\mathbf{P}_{2}\right)$. Seja $(M, q) \in \operatorname{Quad}(A)$ tal que $\operatorname{dim}(q)=n$ e $|\sigma(q)|<n$ para cada $\sigma \in \mathcal{A} s s(A)$.

Desde que $\sigma(q)=\sigma\left(b_{q}\right)$, onde $b_{q}$ é visto como um elemento de $\mathcal{W}(A)$, e $\sigma\left(b_{q}\right) \equiv \operatorname{dim}\left(b_{q}\right) \bmod 2$, temos que $\sigma(q)$ assume somente valores inteiros pares. Logo os possiveis valores de $\sigma(q)$ são $-n+2,-n+4, \ldots, n-4, n-2$. Considere os subconjuntos $\operatorname{de} \mathcal{A s s}(A)$,

$$
Y_{k}=\{\sigma \in \mathcal{A} s s(A) ; \sigma(q)=-n+2 k\},
$$

com $k=1,2, \ldots, n-1$. Claramente $\left\{Y_{k ;} 1 \leq k \leq n-1\right\}$ forma uma partição de $\mathcal{A} s s(A)$, podendo alguns deles serem vazios. De (3.26) temos que $f_{q}: \mathcal{A} s s(A) \rightarrow \mathbb{Z}$, definida por $f_{q}(\sigma)=\sigma(q)$ é uma função contínua e, para cada $k=1, \ldots, n-1, Y_{k}=f_{q}^{-1}(-n+2 k)$, o que mostra que cada $Y_{k}$ é um fechaberto de $\mathcal{A s s}(A)$. Desde que união finita de fechabertos é fechaberto, de $\left(\mathbf{P}_{2}\right)$ obtemos que existem elementos $\beta_{1}, \ldots, \beta_{n-2} \in A^{\star}$ tais que $W\left(\beta_{1}\right)=Y_{1}$, $W\left(\beta_{2}\right)=Y_{1} \cup Y_{2}, \ldots, W\left(\beta_{i}\right)=Y_{1} \cup Y_{2} \cup \ldots \cup Y_{i}, 1 \leq i \leq n-2$. 
Sejam $\beta=\left\langle\beta_{1}, \ldots \beta_{n-2}\right\rangle \in \mathcal{B i l}(A)$ e $\sigma \in Y_{k}$. Desde que $Y_{k} \subseteq W\left(\beta_{i}\right)$, para $k \leq i \leq n-2$ e $Y_{k} \cap W\left(\beta_{i}\right)=\varnothing$, para $1 \leq i \leq k-1$, temos que

$$
\sigma(b)=(k-1)-(n-2-(k-1))=-n+2 k=\sigma\left(b_{q}\right),
$$

para todo $k=1, \ldots, n-1$. Como $\left\{Y_{k} ; k=1, \ldots, n-1\right\}$ é uma partição de $\mathcal{A} s s(A)$, temos que $\sigma(b)=\sigma\left(b_{q}\right)$, para toda $\sigma \in \mathcal{A} s s(A)$.

Seja $q^{\prime}=[1, h]$ como em (2.4). De (3.30) $\sigma\left(q^{\prime}\right)=2$, para toda $\sigma \in \mathcal{A s s}(A)$. Assim, $\sigma\left(b \otimes q^{\prime}\right)=\sigma\left(2 b_{q}\right)=\sigma(2 q)$, para toda $\sigma \in \mathcal{A s s}(A)$. De (3.23) e (3.24) temos que existe um inteiro $m \geq 0$ tal que

$$
2^{m}\left(b \otimes b_{q^{\prime}}\right)=2^{m}\left(2 b_{q}\right), \quad \text { em } \mathcal{W}(A) .
$$

$\operatorname{De}(3.26), 2^{m+3}\left(b \otimes q^{\prime}\right)=2^{m+4} q$ em $\mathcal{W}_{Q}(A)$. Desde que $\operatorname{dim}\left(2^{m+4} q\right)>\operatorname{dim}\left(2^{m+3}\left(b \otimes q^{\prime}\right)\right)$ e vale o Teorema do Cancelamento de Witt para espaços quadráticos, temos que $2^{m+4} q$ é isotrópico, o que implica que $A$ satisfaz $\left(\mathbf{P}_{6}^{\prime}\right)$

Reciprocamente, se $A$ satisfaz $\left(\mathbf{P}_{6}^{\prime}\right)$, para mostrarmos que $A$ satisfaz $\left(\mathbf{P}_{2}\right)$, de (4.3), é suficiente mostrarmos que $A$ satisfaz $\left(\mathbf{P}_{3}\right)$. Para tanto, usando indução sobre o número de unidades, é suficiente mostrarmos que dados $\alpha, \beta \in A^{\star}$, existe $\gamma \in A^{\star}$ tal que

$$
W(\alpha) \cap W(\beta)=W(\gamma)
$$

Considere o espaço quadrático $q=\langle-1, \alpha, \beta, \alpha \beta\rangle \otimes q^{\prime}$, onde $q^{\prime}=[1, h]$ como acima. Dado $\sigma \in \mathcal{A} s s(A)$, temos que $\sigma(\langle\alpha\rangle)= \pm 1, \sigma(\langle\beta\rangle)= \pm 1$ e $\sigma(\langle\alpha \beta\rangle)=\sigma(\langle\alpha\rangle) \sigma(\langle\beta\rangle)$. Logo $\sigma(\langle-1, \alpha, \beta, \alpha \beta\rangle)= \pm 2$, ou seja $\sigma(q)= \pm 4$, para cada $\sigma \in \mathcal{A} s s(A)$.

Como $\operatorname{dim}(q)=8$, de $\left(\mathbf{P}_{6}^{\prime}\right)$ temos que existe um inteiro $m \geq 0$ tal que $2^{m} q$ é isotrópico.

Suponhamos que $|A / \mathfrak{m}| \geq 3$, para todo $\mathfrak{m} \in S p m(A)$. Desde que

$$
2^{m} q=2^{m}\langle-1\rangle \otimes q^{\prime} \perp 2^{m}\langle\alpha, \beta, \alpha \beta\rangle \otimes q^{\prime}
$$

é isotrópico, de [Ba], Theorem(5.2),(iii), temos que existe $\xi \in A^{\star}$ tal que $-\xi$ é representado por $2^{m}\left(-q^{\prime}\right)$ e $\xi$ é representado por $2^{m}\langle\alpha, \beta, \alpha \beta\rangle \otimes q^{\prime}$. Agora, desde que $\operatorname{dim}\left(2^{m}\langle\alpha\rangle \otimes q^{\prime}\right) \geq 2$, $\operatorname{dim}\left(2^{m}\langle\beta\rangle \otimes\langle 1, \alpha\rangle \otimes q^{\prime}\right) \geq 2$ e $\xi$ é representada por $2^{m}\langle\alpha, \beta, \alpha \beta\rangle \otimes q^{\prime}$, temos novamente de [Ba],Theorem(5.2),(ii), que existem $\xi_{1}, \xi_{2}$ unidades de $A$ tais que $\xi_{1}$ é representado por $2^{m}\langle\alpha\rangle \otimes q^{\prime}, \xi_{2}$ é representado por $2^{m}\langle\beta\rangle \otimes\langle 1, \alpha\rangle \otimes q^{\prime}$ e $\xi=\xi_{1}+\xi_{2}$. Tomando $\gamma=\beta^{-1} \xi_{2}$, temos que $\xi=\xi_{1}+\beta \gamma$, com $\gamma$ representado por $2^{m}\langle 1, \alpha\rangle \otimes q^{\prime}$.

Dado $\sigma \in \mathcal{A} s s(A)$, com $\sigma(\langle\alpha\rangle)=1$, temos que $2^{m}\langle\alpha\rangle \otimes q^{\prime}$ é positiva definida com relação à $\sigma$ e, desde que $\xi_{1}$ é representado por $2^{m}\langle\alpha\rangle \otimes q^{\prime}$, de (3.28), temos que $\sigma\left(\left\langle\xi_{1}\right\rangle\right)=1$. 
Conseqüentemente, $W\left(\xi_{1}\right) \subseteq W(\alpha)$. Se esta inclusão é estrita, então existe $\tau \in \mathcal{A} s s(A)$ tal que $\tau(\langle\alpha\rangle)=-1$ e $\tau\left(\left\langle\xi_{1}\right\rangle\right)=1$. Assim, $2^{m}\langle\alpha\rangle \otimes q^{\prime}$ é negativo definido em relação à $\tau$ e representa $\xi_{1} \in A^{*} \operatorname{com} \tau\left(\left\langle\xi_{1}\right\rangle\right)=1$, o que contradiz (3.29). Logo $W\left(\xi_{1}\right)=W(\alpha)$.

De maneira análoga, usando que $\gamma$ é representado por $2^{m}\langle 1, \alpha\rangle \otimes q^{\prime}$, mostra-se que $W(\gamma) \subseteq W(\alpha)$.

Desde que $2^{m}(-q)$ é negativo definido com relação à $\sigma$, para cada $\sigma \in \mathcal{A} s s(A), \mathrm{e}-\xi$ é representado por $2^{m}(-q)$, obtemos de (3.29) que $\sigma(\langle-\xi\rangle)=-1$, isto é, $\sigma(\langle\xi\rangle)=1$, para todo $\sigma \in \mathcal{A} s s(A)$.

Para mostrarmos a inclusão

$$
W(\gamma) \subseteq W(\alpha) \cap W(\beta)
$$

basta mostrarmos que $W(\gamma)$ também está contido em $W(\beta)$. Suponhamos que $W(\gamma) \nsubseteq W(\beta)$. Então existe $\sigma \in W(\gamma)-W(\beta)$, ou seja, existe $\sigma \in \mathcal{A} s s(A)$ tal que $\sigma(\langle\beta\rangle)=1$ e $\sigma(\langle\gamma\rangle)=-1$. Logo $\sigma(\langle\beta \gamma\rangle)=-1$ e, como $\sigma \in W(\gamma) \subseteq W(\alpha)=W\left(\xi_{1}\right)$, temos que $\sigma\left(\left\langle\xi_{1}\right\rangle\right)=-1$. Assim $\sigma\left(\left\langle\xi_{1}\right\rangle \perp\langle\beta \gamma\rangle\right)=-2$. Mas de (3.5) temos que

$$
\left\langle\xi_{1}\right\rangle \perp\langle\beta \gamma\rangle \simeq\langle\xi\rangle \perp\left\langle\xi_{1} \beta \gamma \xi\right\rangle .
$$

Aplicando $\sigma$ em ambos os lados temos $-2=2$, o que é uma contradição. Portanto

$$
W(\gamma) \subseteq W(\alpha) \cap W(\beta) .
$$

Agora seja $\sigma \in W(\alpha) \cap W(\beta)$. Como $W(\alpha)=W\left(\xi_{1}\right)$, temos $\sigma\left(\left\langle\xi_{1}\right\rangle\right)=-1$ e, novamente aplicando $\sigma$ na isometria acima temos

$$
-1+\sigma(\langle\beta \gamma\rangle)=1-\sigma(\langle\beta \gamma\rangle),
$$

implicando $\sigma(\langle\beta \gamma\rangle)=1$. Desde que $\sigma \in W(\beta)$, temos que $\sigma(\langle\gamma\rangle)=-1$, ou seja $\sigma \in W(\gamma)$, o que mostra que $W(\alpha) \cap W(\beta)=W(\gamma)$. Assim, mostramos que se $A$ satisfaz $\left(\mathbf{P}_{6}^{\prime}\right)$ e $|A / \mathfrak{m}| \geq 3$ para todo $\mathfrak{m} \in \operatorname{Spm}(A)$, então $A$ satisfaz $\left(\mathbf{P}_{2}\right)$.

Se $A$ é um anel semilocal que satisfaz a propriedade $\left(\mathbf{P}_{6}^{\prime}\right)$ e tem um ideal maximal $\mathrm{m}$ com $A / \mathrm{m} \cong \mathbb{F}_{2}$, consideremos a extensão cúbica de $A, C=\frac{A[X]}{(f(X))}$, onde $f(X)=X^{3}+6 X^{2}+$ $29 X+1$. Para o anel semilocal $C$ temos que todo corpo residual tem mais que 6 elementos e, seus correspodentes espaços de assinaturas $\mathcal{A} s s(A)$ e $\mathcal{A} s s(C)$ são homeomorfos. Assim $C$ também satisfaz a propriedade $\left(\mathbf{P}_{6}^{\prime}\right)$ e, pelo desenvolvimento acima existe uma unidade $\gamma^{\prime} \in C^{\star}$ tal que

$$
W^{\prime}(\alpha) \cap W^{\prime}(\beta)=W^{\prime}\left(\gamma^{\prime}\right),
$$


onde $W^{\prime}\left(\alpha^{\prime}\right), \operatorname{com} \alpha^{\prime} \in C^{\star}$ formam a subbase de Harrison de $\mathcal{A s s}(C)$. De [Knel] Lemma 4, obtemos que

$$
W(\alpha) \cap W(\beta)=W(\gamma)
$$

$\operatorname{com} \gamma=N_{C / A}\left(\gamma^{\prime}\right) \in A^{*}$, onde $N_{C / A}$ denota a norma da extensão de anéis $C$ de $A$, finalizando assim a demonstração do teorema.

Observação 4.10 Os conceitos de extensão de assinaturas para extensão dos anéis de base, a demonstração de que $\mathcal{A s s}(A)$ e $\mathcal{A s s}(C)$ são espaços topológicos homeomorfos e a demonstração do Lemma 4 de [Knel] utilizadas no final da demonstração do teorema anterior foram necessárias para demonstrarmos o teorema quando $A$ é um anel semilocal que admite corpo residual com 2 elementos. Portanto, desnecessários se assumirmos que $A / \mathfrak{m} \geq 3$, para todo $\mathfrak{m} \in S p m(A)$ ou que $2 \in A^{*}$. Por serem conceitos e resultados de difícil entendimento e, pela limitação de tempo para a conclusão deste trabalho, optamos por não reproduzi-los aqui.

Finalmente para concluirmos a demonstração das equivalências das propriedades topológicas citadas, resta mostrarmos que $\left(\mathbf{P}_{\mathbf{6}}\right)$ e $\left(\mathbf{P}_{\mathbf{6}}^{\prime}\right)$ são equivalentes. Observe que $\left(\mathbf{P}_{\mathbf{6}}^{\prime}\right)$ é a propriedade de Hasse-Minkowiski para espaços quadráticos enquanto que $\left(\mathbf{P}_{6}\right)$ é a propriedade de Hasse-Minkowiski para espaços bilineares.

Teorema 4.11 As propriedades $\left(\mathbf{P}_{6}\right)$ e $\left(\mathbf{P}_{6}^{\prime}\right)$ são equivalentes.

Dem.: Está equivalência segue da definição de espaço quadrático e/ou bilinear indefinido, de (2.14) e de (2.15). 


\section{Referências Bibliográficas}

[A-M] AtiYah, M. F.; Macdonald, I. G., Introduction to Commutative Algebra, University of Oxford, (1969).

[Ba] BaEzA, R., Quadratic Forms Over Semilocal Rings, L. N. M. 655, Springer-Verlag, (1978).

[Bou] Bourbaki, N., Commutative Algebra, Addison-Wesley, (1970).

[Dias] DiAs, I., Formas Quadráticas Sobre LG-Anéis, Tese de Doutorado, IMECCUNICAMP, (1988).

[E-L] Elman R.; Lam, T. Y., Quadratic Forms Over Formally Real Fields and Pythagoream Fields, Amer. J, Math. 94, 1155-1195, (1972).

[Her] Hernstein, I. N., Tópicos de Álgebra, Editora da Universidade de São Paulo e Polígono, (1970).

[Kap] Kaplansky, I., Commutative Rings, Allyn an Bacon, Boston, (1970).

[Kne1] Knebush, M., Remarks on the Paper "Equivalent Topological Properties of the Space of Signatures of a Semilocal Ring" by A. Rosenberg and R. Ware, Publ. Math.; Debrecen, 24, Fasc 1-2, 181-188, (1977).

[Kne2] Knebush, M., Generalization of a Theorem of Artin-Pfister to Arbitrary Semilocal Rings, and Related Topics, Journal of Algebra 36, 46-67, (1975).

[KRW1] Knebush, M.; Rosenberg, A.; Ware, R., Structure of Witt Rings and Quotients of Abelian Group Rings, Amer. J. Math. 94, 119-155, (1972).

[KRW2] Knebush, M.; Rosenberg, A.; Ware, R., Signatures on Semilocal Rings, Journal of Algebra 26, 208-250, (1973). 
[Lam] LAM, T. Y., The Algebraic Theory of Quadratic Forms, University of California, (1973).

[McD] McDonald, B. R., Linear Algebra Over Commulative Rings, Pure and Applicd Math. 87, Marcel Dekker Inc., (1984).

[Mun] Munkres, J. R., Topology, Prentice-Hall Inc., (1975).

[Pie] Pierce, R., Associative Algebras, G. T. M, Springer-Verlag, (1982).

[Pre] Prestel, A., Quadratische Semi-ordnungen und Quadratische Formen, Maht. Z. $133,319-342,(1973)$.

[Reb] Rebolho, D. C., Ideais Primários em Anéis de Witt, Dissertação de Mestrado, ICMC-USP, (2000).

[RW] Rosenberg, A.; Ware, R., Equivalent Topological Properties of the Space of Signatures of a Semilocal Ring, Publ. Math. Debrecen 23, Fasc. 3-4, 283-288, (1976).

[Sch] Scharlau, W., Quadratic and Hermitian Forms, A Series of Comprehensive Studies in Mathematics, Springer-Verlag, (1985).

[Ser] Serre, J. P., A Course in Arithmetic, Springer International Student Edition, Springer-Verlag, (1979). 